\title{
Representations of the Weyl Algebra in Quantum Geometry
}

\author{
Christian Fleischhack ${ }^{1,2}$ \\ 1 Max-Planck-Institut für Mathematik in den Naturwissenschaften, Inselstraße 22-26, \\ 04103 Leipzig, Germany \\ 2 Department Mathematik, Universität Hamburg, Bundesstraße 55, 20146 Hamburg, Germany. \\ E-mail: christian.fleischhack@math.uni-hamburg.de
}

Received: 23 October 2007 / Accepted: 20 April 2008

Published online: 22 October 2008 - (C) The Author(s) 2008. This article is published with open access at Springerlink.com

\begin{abstract}
The Weyl algebra $\mathfrak{A}$ of continuous functions and exponentiated fluxes, introduced by Ashtekar, Lewandowski and others, in quantum geometry is studied. It is shown that, in the piecewise analytic category, every regular representation of $\mathfrak{A}$ having a cyclic and diffeomorphism invariant vector, is already unitarily equivalent to the fundamental representation. Additional assumptions concern the dimension of the underlying analytic manifold (at least three), the finite wide triangulizability of surfaces in it to be used for the fluxes and the naturality of the action of diffeomorphisms - but neither any domain properties of the represented Weyl operators nor the requirement that the diffeomorphisms act by pull-backs. For this, the general behaviour of $C^{*}$-algebras generated by continuous functions and pull-backs of homeomorphisms, as well as the properties of stratified analytic diffeomorphisms are studied. Additionally, the paper includes also a short and direct proof of the irreducibility of $\mathfrak{A}$.
\end{abstract}

\section{Introduction}

Every physical theory requires fundamental mathematical assumptions at the very beginning. It is highly desirable to justify them by even more fundamental axioms that are both mathematically and physically as plausible as possible. In loop quantum gravity, there are a few of such technical prerequisites. First of all, of course, one assumes that all objects are constructed out of parallel transports along graphs in a base manifold of an $S U$ (2) principal fibre bundle (or maybe also using higher dimensional objects like in spin foam theory). This is reasonable by the fact that classical (canonical) gravity is an $S U$ (2) gauge field theory with constraints as discovered by Ashtekar in the mid-80s [1]. Secondly, one needs inputs about the quantization of this classical system. For this, at least the structure of the configuration space $\mathcal{C}$ of all those parallel transports (modulo gauge transforms) has to be fixed. If one wants to use functional integrals for quantization, one is forced to study measures on that space. The usage of parallel transports corresponding to smooth connections only, however, has lead to enormous mathematical 
problems. These could be widely avoided only by including distributional connections as well [2]. Namely, by the assumption that the reductions of the full theory to finitely many degrees of freedom (i.e., parallel transports on a finite graph) are continuous, one finds that the topology of $\mathcal{C}$ is a projective limit topology making $\mathcal{C}$ a compact space. Here, the compactness is induced by that of the underlying structure group $S U$ (2) comprising the values of the parallel transports. This strategy can be reused to find natural measures on $\mathcal{C}$ - one simply uses the assumption that the restrictions of the theory to finite graphs push forward the measure on $\mathcal{C}$ to the Haar measures on the finite powers of $S U(2)$. This leads to the Ashtekar-Lewandowski measure $\mu_{0}$ [6]. Of course, this measure is "natural", since the Haar measure on a Lie group is "natural" as well. However, this is at most a mathematical statement or a statement of beauty. The deeper question behind is how one can justify this choice by mathematical physics arguments.

1.1. Early attempts. For the first time, this problem has been raised by Sahlmann [32]. He considered the class of measures on $\mathcal{C}$ that are absolutely continuous w.r.t. $\mu_{0}$, and realized [32,33] that (up to some additional technical assumptions) only $\mu_{0}$ allows for a diffeomorphism invariant measure such that the flux variables are represented as operators on the corresponding $L_{2}$ space. Although these results were proven for the case of a $U(1)$ gauge theory, they have been expected to hold also for the case of a general compact structure Lie group G. Moreover, it suggests that the diffeomorphism invariance of gravity together with its full phase space description could be responsible for the uniqueness of $\mu_{0}$. The situation is similar to ordinary quantum mechanics. There, the Stone-von Neumann theorem [11] tells us that there is (up to equivalence) precisely one irreducible regular representation of the Weyl algebra generated by the exponentiated position and momentum operators together with their Poisson relations. In the standard Schrödinger representation on $L_{2}(\mathbb{R}, \mathrm{d} x)$, these unitary operators are given by

$$
\left[\mathrm{e}^{\mathrm{i} \pi \hat{x}} \psi\right](x)=\mathrm{e}^{\mathrm{i} \pi x} \psi(x) \text { and }\left[\mathrm{e}^{\mathrm{i} \xi \hat{p}} \psi\right](x)=\psi(x+\xi) .
$$

In loop quantum gravity, on the other hand, the connections are the generalized positions and the densitized dreibein fields are the generalized momenta. Exponentiation here includes also smearing: Connections are smeared along one-dimensional objects (i.e., paths) and exponentiated to give parallel transports - dreibeine along one-codimensional objects (i.e., hypersurfaces) to give flux variables. Now, one possible (even irreducible and regular) representation for the corresponding Weyl algebra $\mathfrak{A}$ is given by multiplication and translation operators, respectively, on $L_{2}$ functions on $\mathcal{C}$ w.r.t. the Ashtekar-Lewandowski measure. All that suggests that maybe this representation $\pi_{0}$ is even uniquely determined as well by certain reasonable assumptions. Sahlmann and Thiemann [35,34], supported by results of Lewandowski and Okołów [30] (see also [25] for further discussion), had argued that $\pi_{0}$ may be the only irreducible, regular and diffeomorphism invariant representation of $\mathfrak{A}$. Despite the progress given by these papers, there had remained many open points, both technically and conceptually. A conceptual one concerned the domain properties of the represented operators. In fact, all results for non-abelian structure groups in [35] relied crucially on the fact that the self-adjoint generators of both the represented and the non-represented unitary operators share a certain, but not naturally given common dense domain. Another issue regarding the smoothness properties of the diffeomorphisms will be discussed below.

\footnotetext{
1 Of course, any refinement of this topology leads to continuous reductions again. However, for simplicity, one ignores this possibility.
} 
1.2. Achievements of the present paper. The situation above has described the status some five years ago. The goal of our present paper is now to give a complete and rigorous proof of a Stone-von Neumann-like theorem in quantum geometry avoiding most of these problems. More precisely, we will show that every regular representation of $\mathfrak{A}$ that has a cyclic and diffeomorphism invariant vector, is unitarily equivalent to the fundamental representation $\pi_{0}$, provided the action of diffeomorphisms satisfies some rather mild condition. The main conceptual achievements of our theorem, in comparison to [35], are the following:

- There are no longer any requirements concerning the domains of the operators in the game. This will be possible, since we consequently, from the very beginning, work with the exponentiated fluxes only. At no point, will we use their self-adjoint generators. There is only one issue, where we use the relation between operators and their generators. This will concern one-parameter subgroups in a compact Lie group in order to get some estimate for certain products in it. However, we will completely leave this infinitesimal arena before going back to the Weyl algebra level.

- The requirements concerning the representations of the diffeomorphisms are drastically weakened. In [35], it had to be assumed that these are represented via pull-backs and respect the decomposition of the representation restricted to $C(\mathcal{C})$ into cyclic generators. In particular, one had to assume that each of these components contains a diffeomorphism invariant cyclic vector. As to be discussed at the end of the paper, a priori these requirements drastically reduce the measures allowed in these decompositions. We will now be able to show that this assumption can be replaced by a weaker one. We only require that coinciding addends in the decomposition share the same representation of diffeomorphisms if at least one addend is diffeomorphism invariant.

- Moreover, we will be able to clarify the particular class of diffeomorphisms to be used. Analytic diffeomorphisms are unsatisfactory from two points of view: Physically, they contradict the notion of locality, i.e., if we transform some set in the space(-time) manifold locally, then we transform this manifold even globally. Mathematically, they are not flexible enough as well, i.e., it will often be very difficult, if not impossible, to locally map objects onto each other under very rigid conditions, as we will see below. Therefore, we are forced to extend the class of isomorphisms. In fact, it will be manageable to use stratified analytic diffeomorphisms, slightly modifying the similar structures in, e.g., [29,21,10]. This, at the same time, leads to a natural extension of the surfaces used to define the Weyl operators, from analytic submanifolds to semianalytic sets. However, this is not a severe extension, since every semianalytic set can be stratified into a locally finite set of analytic submanifolds being mutually disjoint, i.e., having commuting Weyl operators.

1.3. Idea of the proof. Let us very shortly outline the proof of the uniqueness theorem. As usual (see, e.g., [35]), the restriction of any representation $\pi$ of a Weyl-like algebra to the continuous functions, can be decomposed into (w.r.t. $C(\mathcal{C})$ ) cyclic ones. These are always the canonical representations on some $L_{2}\left(\mathcal{C}, \mu_{v}\right)$ with appropriate measures $\mu_{v}$ on $\mathcal{C}$. Assuming that $\pi$ contained a cyclic vector having some invariance property, we may find such a decomposition, such that one of the constant vectors $\mathbf{1}_{v} \in L_{2}\left(\mathcal{C}, \mu_{v}\right)$ has these properties as well. Then, being the first step where we use the particular structures of quantum geometry, regularity and diffeomorphism invariance imply that this $\mu_{v}$ is the Ashtekar-Lewandowski measure. Now, being the second step relying 
on quantum geometry, we may show that certain Weyl operators are diffeomorphism conjugate to their adjoints. By general arguments, using the two properties above and adding invariance and cyclicity of $\mathbf{1}_{v}$, we prove that $\pi$ equals (up to unitary equivalence) the fundamental representation of $\mathfrak{A}$.

1.4. Comparison with LOST paper. While this paper was prepared, Lewandowski, Okołów, Sahlmann and Thiemann (LOST) were working on a similar problem for the holonomy-flux $*$-algebra. This algebra is given if the fluxes themselves are considered together with the continuous functions on $\mathcal{C}$. Some time after the present article had been sent to the arxiv, the four-men paper [26] has been finished and appeared there as well. In this subsection, we are going to compare the corresponding results.

As already mentioned, the most striking difference between the two approaches lies in the algebra: We use both exponentiated positions and momenta, but LOST exponentiate positions only and keep the fluxes non-exponentiated. Consequently, LOST investigate the holonomy-flux algebra, a *-algebra, but we consider the Weyl algebra - a $C^{*}$-algebra. Here the exponentiated fluxes are implemented as unitaries, whereas LOST study implicitly their self-adjoint generators being, of course, unbounded. The price to pay is that, in contrast to our case, LOST have to get rid of the persistent domain problems. This is done very directly using a state, since that -via GNS-guarantees the existence of a common dense domain for all the operators. By construction, this domain is spanned by the cylindrical functions on $\mathcal{C}$. On the other hand, we only assume that the Weyl operators are continuously represented w.r.t. their smearing. This means that each corresponding one-parameter subgroup has some self-adjoint generator. If this was not the case, it is expected that then there exist other diffeoinvariant representations of the Weyl algebra. Nevertheless, note that our regularity assumption for each single oneparameter subgroup is much weaker than that of the existence of a certain common dense domain for all generators as in the LOST case. Indeed, our assumption follows from the LOST requirements: The GNS construction implies that, given a state, the $*$-invariant fluxes become symmetric operators. As it turns out, they are even self-adjoint. Hence they generate weakly continuous one-parameter subgroups.

All that seems to show that our result is much stronger than that of LOST. However, there will be an additional assumption made in our paper only: the diffeomorphisms are implemented naturally. Until now, by no means, neither the relevance of this requirement nor its possible counterpart in the LOST paper is clear. However, while, as a matter of principle, it cannot be expected that the domain assumptions above can be dropped by LOST, we do hope that the naturality condition can be shown obsolete sometime.

The remaining differences are, from our point of view, secondary. Let us only sketch a few of them. The technical advantage of the $*$-algebra case is the linearity of the fluxes w.r.t. the smearing, which enables LOST to use the scalar-product trick by Okołów. At the same time, LOST have to use compactly supported smearing functions. We, on the other hand, are confined to (up-to-gauge) constant smearings, although there is some hope to relax that. Since compactly supported smearings mean that one can restrict oneself to "nice" parts of the surfaces and forget about near-boundary regions, LOST -in contrast to us- did not have to assume that the surfaces are (widely) triangulizable.

Rather similar are the general assumptions concerning smoothness. The striking idea that underlies both investigations is that stratified analytic objects comprise both the advantages of analyticity and those of locality. Only the implementation somewhat differs. Both are influenced by the notion of semianalyticity introduced mainly by Łojasiewicz, but - for simplicity - we mostly study these structures on a given analytic 
Table 1. Comparison between LOST and Fleischhack

\begin{tabular}{|c|c|c|}
\hline & LOST & Fleischhack \\
\hline theory & gauge field theory & gauge field theory \\
\hline geometric ingredients & $\begin{array}{l}\text { principal fibre bundle } P \\
\text { - structure group } G \\
\text {. base manifold } M\end{array}$ & $\begin{array}{l}\text { principal fibre bundle } P \\
\text { - structure group } G \\
\text {. base manifold } M\end{array}$ \\
\hline smoothness & $\begin{array}{l}\text { stratified analytic } \\
\cdot C^{\boldsymbol{k}} \\
\cdot \text { semianalytic }\end{array}$ & $\begin{array}{l}\text { stratified analytic } \\
\cdot C^{\mathbf{0}} \\
\cdot \text { semi- or subanalytic }\end{array}$ \\
\hline basic assumptions & $\begin{array}{l}G \text { compact connected Lie } \\
\cdot M \text { stratified analytic } \\
\cdot \operatorname{dim} M \geq \mathbf{2}\end{array}$ & $\begin{array}{l}\cdot G \text { compact connected Lie } \\
\cdot M \text { analytic } \\
\cdot \operatorname{dim} M \geq \mathbf{3}\end{array}$ \\
\hline diffeomorphisms & stratified analytic & stratified analytic \\
\hline $\begin{array}{l}\text { positions } \\
\cdot \text { exponentiated } \\
\cdot \text { smeared along }\end{array}$ & $\begin{array}{l}\text { connections } \\
\cdot \text { yes } \\
\text {. paths }\end{array}$ & $\begin{array}{l}\text { connections } \\
\text { - yes } \\
\text {. paths }\end{array}$ \\
\hline paths & stratified analytic & stratified analytic \\
\hline $\begin{array}{l}\text { momenta } \\
\cdot \text { exponentiated } \\
\cdot \text { smeared along }\end{array}$ & $\begin{array}{l}\text { fluxes } \\
\cdot \text { no } \\
\cdot \text { surfaces }\end{array}$ & $\begin{array}{l}\text { fluxes } \\
\cdot \text { yes } \\
\cdot \text { surfaces }\end{array}$ \\
\hline surfaces & $\begin{array}{l}\text { stratified analytic } \\
\text { - open } \\
\text { - codimension } 1 \\
\text {. - }\end{array}$ & $\begin{array}{l}\text { stratified analytic } \\
\cdot \text { open } \\
\cdot \text { codimension } 1+ \\
\text {. widely triangulizable }\end{array}$ \\
\hline smearing functions & $\begin{array}{l}\text { stratified analytic } \\
\text { compactly supported }\end{array}$ & $\begin{array}{l}\text { stratified analytic } \\
\text { constant on strata }\end{array}$ \\
\hline algebra & holonomy-flux algebra & Weyl algebra \\
\hline type & $*$-algebra & $C^{*}$-algebra \\
\hline \multirow[t]{2}{*}{ generators } & $\begin{array}{l}\text { positions } \\
\text { cylindrical functions on } \mathcal{C}\end{array}$ & $\begin{array}{l}\text { positions } \\
\text { c continuous functions on } \mathcal{C}\end{array}$ \\
\hline & $\begin{array}{l}\text { momenta } \\
\text { - weak derivatives of } \\
\text { pull-backs of left/right } \\
\text { translations on } \mathcal{C}\end{array}$ & $\begin{array}{l}\text { momenta (unitary) } \\
\text { pull-backs of left/right } \\
\text { translations on } \mathcal{C}\end{array}$ \\
\hline$\overline{\overline{\text { uniqueness }}}$ & state & representation \\
\hline assumed cyclicity & cyclic invariant vector & cyclic invariant vector \\
\hline domain assumptions & $\begin{array}{l}\text { common dense domain: } \\
\text { cylindrical functions }\end{array}$ & - \\
\hline$\overline{\text { regularity assumptions }}$ & - & regularity w.r.t. smearing \\
\hline add'l assumptions & - & natural diffeo action \\
\hline required invariance & $\begin{array}{l}\text { all bundle automorphisms } \\
\text { - diffeomorphisms } \\
\text {. gauge transformations }\end{array}$ & $\begin{array}{l}\text { some bundle automorphisms } \\
\text { - some diffeomorphisms } \\
\text {. _ }\end{array}$ \\
\hline
\end{tabular}

manifold, whereas LOST define semianalytic structures in a more categorical way. Nevertheless, essentially all of our considerations should be directly transferable to the LOST framework and vice versa. There should also be no significant changes if we required semianalyticity to include not only continuity at the boundaries, but also $C^{k}$ as in the LOST regime. Only in the $C^{\infty}$ case, this is not completely clear.

Finally, we summarize our comparison in Table 1 . Note that there we slightly modify the notions used in the respective article to better explain coincidences and differences.

1.5. Further developments. Both the LOST and the present paper originate from the quest for a quantum gravity theory. Therefore, as said above, its main application 
concerns an $S U$ (2)-gauge field theory over a three-dimensional manifold $M$ (i.e., some Cauchy surface) with diffeomorphism invariance as a fundamental symmetry. All the results contain, of course, this case, but go much beyond. Nevertheless, some related questions are still unsolved. For instance, what about theories with other symmetries or another field content? First results have been obtained for homeomorphism invariant scalar field theories [23,24]. Here, it turned out, that there are indeed other states, labelled by the Euler characteristics, i.e., algebraic-topological properties of the hypersurfaces. Another approach currently under investigation, has been taken by Bahr and Thiemann [9] extending the diffeomorphism group symmetry to general automorphisms of the path groupoid.

1.6. Structure of the article. To finish the introduction, let us briefly outline the present paper. In Sect. 2 we start with a general investigation of $C^{*}$-algebras that are generated by the continuous functions on a compact Hausdorff space $X$ and by pull-backs of homeomorphisms of $X$. Afterwards, we switch over to quantum geometry. Since we would like to make the theory applicable to weaker smoothness classes the paths are required to belong to, we generalize the notion of oriented surfaces introducing quasi-surfaces and intersection functions in Sect. 3. Then, in Sect. 4, the Weyl algebra of quantum geometry is defined and the assumed structures regarding paths, hypersurfaces, diffeomorphisms etc. are fixed. After presenting a pretty short and direct proof for the irreducibility of the Weyl algebra in Sect. 5, we study the theory of stratified diffeomorphisms in detail in Sect. 6. The main result on the uniqueness of representations is then contained in Sect. 7, including a discussion of the assumptions made and the extensions possible.

\section{General Setting}

Let $X$ be a compact Hausdorff space and $\operatorname{Homeo}(X)$ be the set of all homeomorphisms of $X$. Given some $\xi \in \operatorname{Homeo}(X)$, its pull-back to $C(X)$ is denoted by $w_{\xi}$ or, as usual, $\xi^{*}$. Correspondingly, for every $\mathcal{H} \subseteq \operatorname{Homeo}(X)$, the set $\mathcal{W}_{\mathcal{H}} \equiv \mathcal{H}^{*} \subseteq \operatorname{Homeo}^{*}(X)$ contains precisely the pull-backs of all elements in $\mathcal{H}$. The other way round, given some pull-back $w \in$ Homeo* $^{*}(X)$, the corresponding homeomorphism is denoted by $\xi_{w}$, i.e., we have $\xi_{w}^{*}=w$. Analogously, $\mathcal{H}_{\mathcal{W}} \subseteq \operatorname{Homeo}(X)$ is defined for all $\mathcal{W} \subseteq \operatorname{Homeo}^{*}(X)$. Moreover, we denote by $\langle\mathcal{W}\rangle$ the (abstract) subgroup of Homeo* $(X)$ generated by $\mathcal{W}$ and define, analogously, $\langle\mathcal{H}\rangle$. Obviously, $\left\langle\mathcal{H}_{\mathcal{W}}\right\rangle=\mathcal{H}_{\langle\mathcal{W}\rangle}$ and $\left\langle\mathcal{W}_{\mathcal{H}}\right\rangle=\mathcal{W}_{\langle\mathcal{H}\rangle}$. Next, for every measure ${ }^{2} \mu$ on $X$, we denote by $\mathcal{H}(\mu)$ the set of all homeomorphisms on $X$ leaving $\mu$ invariant. Clearly, $\langle\mathcal{H}(\mu)\rangle=\mathcal{H}(\mu)$. Moreover, every $w \in \mathcal{W}_{\mathcal{H}(\mu)}$ extends naturally to a unitary operator on $L_{2}(X, \mu)$, again denoted by $w$. By $w(f \psi)=w(f) w(\psi)$ for all $f \in C(X), \psi \in L_{2}(X, \mu)$ and $w \in \mathcal{W}_{\mathcal{H}(\mu)}$, we have $w \circ f \circ w^{-1}=w(f)$ as operators in $\mathcal{B}\left(L_{2}(X, \mu)\right)$. Sometimes, we will extend the notion to operators: $w_{1}\left(w_{2}\right):=$ $w_{1} \circ w_{2} \circ w_{1}^{-1}$ for $w_{1}, w_{2} \in \mathcal{W}_{\mathcal{H}(\mu)}$. Finally, let $\mathfrak{A}(\mathcal{W}, \mu)$ denote the $C^{*}$-subalgebra in $\mathcal{B}\left(L_{2}(X, \mu)\right.$ ) generated by $C(X)$ and $\mathcal{W} \subseteq \mathcal{W}_{\mathcal{H}(\mu)}$, and let $\pi_{0}$ be the identical (or fundamental) representation of $\mathfrak{A}(\mathcal{W}, \mu)$ on $L_{2}(X, \mu)$.

Lemma 2.1. For every $\mathcal{W} \subseteq \mathcal{W}_{\mathcal{H}(\mu)}$, the subalgebra spanned by all products $f \circ w$ with $f \in C(X)$ and $w \in\langle\mathcal{W}\rangle$ is dense in $\mathfrak{A}(\mathcal{W}, \mu)$.

\footnotetext{
${ }^{2}$ If not stated otherwise, by a measure we always mean a normalized regular Borel measure.
} 
Proof. Since $w \circ f=w(f) \circ w$ for all $w \in\langle\mathcal{W}\rangle$ and $f \in C(X)$,

$$
\begin{aligned}
& f_{1} \circ w_{1} \circ f_{2} \circ w_{2} \circ \cdots \circ w_{k} \circ f_{k+1} \\
& \quad=\left(f_{1} \cdot w_{1}\left(f_{2}\right) \cdots \cdots w_{1}\left(w_{2}\left(\ldots\left(w_{k}\left(f_{k+1}\right)\right) \ldots\right)\right)\right) \circ\left(w_{1} \circ \cdots \circ w_{k}\right)
\end{aligned}
$$

is always in $C(X) \circ\langle\mathcal{W}\rangle$. Moreover, with $f$, also $f^{*} \equiv \bar{f}$ is in $C(X)$, and with $w$, also $w^{*}=w^{-1}$ is in $\langle\mathcal{W}\rangle$. Therefore, the span of $C(X) \circ\langle\mathcal{W}\rangle$ equals the $*$-subalgebra of $\mathcal{B}\left(L_{2}(X, \mu)\right)$ generated by $C(X)$ and $\mathcal{W}$.

Throughout the whole section, let $\mu$ be some arbitrary, but fixed measure on $X$.

2.1. First-step decomposition. Since every representation of a $C^{*}$-algebra is the direct sum of a zero representation and a non-degenerate one, we may restrict ourselves to non-degenerate representations in the following.

Lemma 2.2. Fix some $\mathcal{W} \subseteq \mathcal{W}_{\mathcal{H}(\mu)}$ and let $\pi$ be a non-degenerate representation of $\mathfrak{A}(\mathcal{W}, \mu)$ on some Hilbert space $\mathfrak{H}$.

Then there are measures $\mu_{v}$ on $X$ with $v$ running over some (not necessarily countable) index set $\mathrm{N}$, such that $\left.\pi\right|_{C(X)}$ is unitarily equivalent to the direct-sum representation $\bigoplus_{v} \pi_{\mu_{v}}$, where $\pi_{\mu_{v}}$ denotes the canonical representation of $C(X)$ on $L_{2}\left(X, \mu_{v}\right)$ by multiplication operators. Moreover, these measures may be chosen, such that two of them are equal if they are equivalent (w.r.t. absolute continuity).

Proof. Every non-degenerate representation of a $C^{*}$-algebra is (up to unitary equivalence) the direct sum of cyclic representations [12]. The first assertion now follows, because every cyclic representation of $C(X)$ is equivalent to the canonical representation on $L_{2}\left(X, \mu_{v}\right)$ by multiplication operators for some regular Borel measure $\mu_{v}$ [36]. Note that $\left.\pi\right|_{C(X)}$ is non-degenerate by $1 \in C(X)$. Since measures on $X$ are equivalent w.r.t. absolute continuity iff the corresponding canonical representations are equivalent [36], we get the proof.

Definition 2.1. A decomposition $\bigoplus_{\nu} \pi_{\mu_{v}}$ as given in Lemma 2.2 is called first-step decomposition of $\pi$.

Sometimes we write $\left(\mu_{\nu}\right)_{v \in \mathrm{N}}$ or shortly $\boldsymbol{\mu}$ to characterize such a decomposition. Moreover, if the particular $\mathcal{W}$ is not important, we will consider first-step decompositions without any reference to some $\pi$.

Definition 2.2. A first-step decomposition is called short iff $\mathrm{N}$ consists of a single element.

Remark. First-step decompositions are not at all unique. In fact, consider a short one with $\mu_{v}=\mu$ and choose $U \subseteq X$ with $0<\mu(U)<1$. Decomposing any $\psi \in \mathfrak{H}$ into $\psi=1_{U} \psi+1_{X \backslash U} \psi$ with $1_{U}$ being the characteristic function on $U$, we get a first-step decomposition $\pi_{\mu_{U}} \oplus \pi_{\mu_{X \backslash U}}$. Here, $\mu_{U}$ is the normalization of $1_{U} \odot \mu$.

In the following, given some representation $\pi$ of $\mathfrak{A}(\mathcal{W}, \mu)$ on $\mathfrak{H}$, we will usually assume that $\left.\pi\right|_{C(X)}$ equals (one of) its first-step decomposition(s). Moreover, we usually write shortly $\pi_{v}$ instead of $\pi_{\mu_{v}}$. By $\|\cdot\|_{\mu_{v}}$ we denote the norm on $L_{2}\left(X, \mu_{v}\right)=: \mathfrak{H}_{v}$ and by $P_{v}$ the respective orthogonal projector mapping $\mathfrak{H}$ to $\mathfrak{H}_{\nu}$. In particular, we have $\|\pi(f) \psi\|_{\mathfrak{H}}^{2}=\sum_{v}\left\|f \cdot P_{\nu} \psi\right\|_{\mu_{v}}^{2}$ for all $f \in C(X)$ and $\psi \in \mathfrak{H}$. Next, let $I_{v}: \mathfrak{H}_{v} \longrightarrow \mathfrak{H}$ 
denote the (norm-preserving) canonical embedding of $\mathfrak{H}_{v}$ into $\mathfrak{H}$ and set $\mathbf{1}_{v}:=I_{v}(\mathbf{1})$, where $\mathbf{1}$ is seen not only as an element in $C(X)$, but in $\mathfrak{H}_{v}$ as well. Anyway, often we will simply drop $I_{\nu}$. Analogously, we do not explicitly mark the transition from continuous functions to their classes in $L_{2}$, when calculating scalar products. Note, however, that $C(X)$ is, in general, not embedded into $L_{2}\left(X, \mu_{v}\right)$. Let, e.g., $\mu_{v}$ be the Dirac measure at some point in $X$, then the image of $C(X)$ is isomorphic to $\mathbb{C}$. Therefore, one has to be careful when operating with pull-backs of homeomorphisms that do not leave $\mu_{v}$ invariant. Finally, for $\mu_{\nu_{1}}=\mu_{v_{2}}$ we denote the canonical isomorphism mapping $I_{\nu_{1}}\left(\mathfrak{H}_{\nu_{1}}\right)$ to $I_{\nu_{2}}\left(\mathfrak{H}_{\nu_{2}}\right)$ by $I_{\nu_{2}}^{\nu_{1}}$.

Definition 2.3. Let $\mathcal{W}$ be a subset of $\mathcal{W}_{\mathcal{H}(\mu)}$ and let $\pi$ be some representation of $\mathfrak{A}(\mathcal{W}, \mu)$ on some Hilbert space $\mathfrak{H}$.

A vector $\psi \in \mathfrak{H}$ is called $\mathcal{W}$-invariant iff $\pi(w) \psi=\psi$ for all $w \in \mathcal{W}$.

Note that we tacitly assume some information about $\pi$ to be given when we speak on invariance w.r.t. some $\mathcal{W}$. This will avoid some cumbersome notation when we study equivalent representations.

Lemma 2.3. Let $\mathcal{W}$ and $\mathcal{W}^{\prime}$ be subsets of $\mathcal{W}_{\mathcal{H}(\mu)}$, let $\pi^{\prime}$ be a representation of $\mathfrak{A}(\mathcal{W} \cup$ $\left.\mathcal{W}^{\prime}, \mu\right)$ on some Hilbert space $\mathfrak{H}$, and let $\psi \in \mathfrak{H}$ be a $\mathcal{W}^{\prime}$-invariant vector.

Then there is a first-step decomposition $\bigoplus_{v \in \mathrm{N}} \pi_{\mu_{v}}$ of $\pi^{\prime}$ and some $v \in \mathrm{N}$, such that $\mathbf{1}_{v}$ is a $\mathcal{W}^{\prime}$-invariant vector. If, moreover, $\psi$ is cyclic for $\left.\pi^{\prime}\right|_{\mathfrak{A}(\mathcal{W}, \mu)}$, then $\mathbf{1}_{v}$ may be chosen cyclic as well.

Proof. Define $\mathfrak{H}_{v}:=\overline{\pi^{\prime}(C(X)) \psi} \subseteq \mathfrak{H}$. Then both $\mathfrak{H}_{v}$ and $\mathfrak{H}_{v}^{\perp}$ are invariant w.r.t. $\pi^{\prime}(C(X))$. Since $\mathfrak{H}_{v}^{\perp}$ is non-degenerate (if not zero), the projection of $\left.\pi^{\prime}\right|_{C(X)}$ to $\mathfrak{H}_{v}^{\perp}$ is (up to equivalence) some direct sum $\bigoplus_{v^{\prime} \in \mathrm{N}^{\prime}} \pi_{\mu_{v^{\prime}}}$ of cyclic representations of $C(X)$. Since, on the other hand, $\left.\pi^{\prime}\right|_{C(X)}$ is cyclic on $\mathfrak{H}_{v}$, it is equivalent to the canonical representation $\pi_{\mu_{v}}$ of $C(X)$ on some $L_{2}\left(X, \mu_{v}\right)$, whereas the corresponding intertwiner maps $\psi$ to $\mathbf{1}_{v}$. Now, by construction, $\pi_{\mu_{v}} \oplus \bigoplus_{\nu^{\prime} \in \mathrm{N}^{\prime}} \pi_{\mu_{\nu^{\prime}}}$ is a first-step decomposition of $\pi^{\prime}$. Moreover, the $\mathcal{W}^{\prime}$-invariance of $\psi$ translates into that of $\mathbf{1}_{v}$ and the cyclicity, if given, as well.

Now, throughout the whole Sect. 2, we let $\mathcal{W}$ and $\mathcal{W}^{\prime}$ be some arbitrary subsets of $\mathcal{W}_{\mathcal{H}(\mu)}$, whereas $w^{\prime}(\mathcal{W}) \subseteq \mathcal{W}$ for all $w^{\prime} \in \mathcal{W}^{\prime}$. Note that we do not assume that they are fixed once and for all, i.e., they may be changed from one statement to the other. Next, $\pi$ and $\pi^{\prime}$ are always non-degenerate representations of $\mathfrak{A}(\mathcal{W}, \mu)$ and $\mathfrak{A}\left(\mathcal{W} \cup \mathcal{W}^{\prime}, \mu\right)$, respectively, on some Hilbert space $\mathfrak{H}$, where $\pi$ is the restriction of $\pi^{\prime}$ to $\mathfrak{A}(\mathcal{W}, \mu) .{ }^{3} \mathrm{We}$ let $\bigoplus_{v} \pi_{\mu_{v}}$ be a fixed first-step decomposition of $\pi$ on $\mathfrak{H}=\bigoplus_{v} \mathfrak{H}_{v}=\bigoplus_{v} L_{2}\left(X, \mu_{v}\right)$ and usually set $\pi_{v}:=\pi_{\mu_{v}}$ for simplicity. Note that every first-step decomposition of $\pi$ is also some for $\pi^{\prime}$ and vice versa, since $\pi$ and $\pi^{\prime}$ coincide on $\mathfrak{A}(\mathcal{W}, \mu)$ containing $C(X)$. Moreover, if there is some $\mathcal{W}^{\prime}$-invariant (and $\pi$-cyclic) vector, then we assume that there is some $v \in \mathrm{N}$, such that $\mathbf{1}_{v}$ is $\mathcal{W}^{\prime}$-invariant (and $\pi$-cyclic). Note that this does not contradict the assumption above that measures in a first-step decomposition are equal if they are equivalent. Finally, in order to fix a home for the one-parameter subgroups in $\mathcal{W}$ introduced later, we fix some subset $\mathcal{R}$ in the set $\operatorname{Hom}(\mathbb{R}, \mathcal{W})$ of homomorphisms from $\mathbb{R}$ to $\mathcal{W}$.

\footnotetext{
3 Often, we will not refer to $\pi^{\prime}$ at all. Then, in general, we tacitly set $\mathcal{W}^{\prime}=\varnothing$ and $\pi^{\prime}=\pi$.
} 
2.2. $\pi_{v}$-Scalars and $\pi_{v}$-Units.

Definition 2.4. An element $w \in \mathcal{W}$ is called

- $\pi_{v}$-scalar iff $P_{v} \pi(w) \mathbf{1}_{v}=c \mathbf{1}$ for some $c \in \mathbb{C}$;

- $\pi_{v}$-unit iff $P_{v} \pi(w) \mathbf{1}_{v}=\mathbf{1}$.

Analogously, we define these properties for $w^{\prime} \in \mathcal{W}^{\prime}$. Since $w$ is unitary, we have

Lemma 2.4. $\mathbf{1}_{v}$ is $\pi(w)$-invariant $\Longleftrightarrow w$ is a $\pi_{v}$-unit $\Longleftrightarrow w^{*}$ is a $\pi_{v}$-unit.

Corollary 2.5. Any finite product of $\pi_{v}$-units is a $\pi_{v}$-unit.

Lemma 2.6. If $\mathbf{1}_{v}$ is $\pi(w)$-invariant, then $\mathfrak{H}_{v}$ and $\mathfrak{H}_{v}^{\perp}$ are $\pi(w)$-invariant.

Proof. Fix some $\psi_{v} \in \mathfrak{H}_{v}$ and recall that $\mathbf{1}_{v}$ is cyclic for $\pi_{v}$, i.e., for every $\varepsilon>0$ there is some $f \in C(X)$ with $\left\|\pi(f) \mathbf{1}_{v}-\psi_{v}\right\|<\varepsilon$.

Since $w$ is a $\pi_{v}$-unit, we have $\pi(w) \pi(f) \mathbf{1}_{v}=\pi(w(f)) \mathbf{1}_{v} \in \mathfrak{H}_{v}$. By unitarity of $w$, we get $\left\|\pi(w) \psi_{v}-\pi(w) \pi(f) \mathbf{1}_{v}\right\|<\varepsilon$, i.e., $\operatorname{dist}\left(\pi(w) \psi_{\nu}, \mathfrak{H}_{\nu}\right)<\varepsilon$ for all $\varepsilon>0$. Hence, $\pi(w) \psi_{v} \in \mathfrak{H}_{v}$.

The invariance of $\mathfrak{H}_{v}^{\perp}$ follows from the unitarity of $\pi(w)$.

Corollary 2.7. If each $w \in \mathcal{W}$ is a $\pi_{v}$-unit, then the restriction of $\pi$ to $\mathfrak{H}_{v}$ is cyclic.

Proof. Since every $\pi_{\nu}$-unit leaves $\mathfrak{H}_{\nu}$ invariant, $\pi(\mathfrak{A}(\mathcal{W}, \mu))$ leaves $\mathfrak{H}_{v}$ invariant. Since $\mathbf{1}_{v}$ is already cyclic on $\mathfrak{H}_{v}$ for $\pi$ restricted to $C(X) \subseteq \mathfrak{A}(\mathcal{W}, \mu)$, we get the assertion.

Lemma 2.8. Let $w \in \mathcal{W}$ be a $\pi_{v}$-scalar and assume $\psi_{0}:=\left(\mathbf{1}-I_{\nu} P_{\nu}\right) \pi(w) \mathbf{1}_{v} \neq 0$. Define $\psi$ to be the normalization of $\psi_{0}$, and let $\mathfrak{H}_{\psi}$ be the completion of $\pi(C(X)) \psi$. Finally, assume that $\mu_{v}$ is $\xi_{w}$-invariant, i.e., $w \in \mathcal{W}_{\mathcal{H}\left(\mu_{v}\right)}$.

Then the restriction of $\left.\pi\right|_{C(X)}$ to its invariant subspace $\mathfrak{H}_{\psi}$ is equivalent to the canonical representation of $C(X)$ on $L_{2}\left(X, \mu_{v}\right)$. Moreover, $\mathfrak{H}_{v}$ and $\mathfrak{H}_{\psi}$ are orthogonal.

Proof. Of course, by definition, $\mathfrak{H}_{\psi}$ is invariant w.r.t. $\pi(C(X))$. Let now $f_{1}$ and $f_{2}$ be in $C(X)$. Then, by unitarity of $\pi(w)$ and $\xi_{w}$-invariance of $\mu_{v}$, we have

$$
\begin{aligned}
& \left\langle\pi\left(f_{1}\right) \psi_{0}, \pi\left(f_{2}\right) \psi_{0}\right\rangle_{\mathfrak{H}} \\
& =\left\langle\pi\left(f_{1}\right)\left(\mathbf{1}-I_{\nu} P_{\nu}\right) \pi(w) \mathbf{1}_{\nu}, \pi\left(f_{2}\right)\left(\mathbf{1}-I_{\nu} P_{\nu}\right) \pi(w) \mathbf{1}_{\nu}\right\rangle_{\mathfrak{H}} \\
& =\left\langle\left(\mathbf{1}-I_{\nu} P_{\nu}\right) \pi\left(f_{1}\right) \pi(w) \mathbf{1}_{\nu},\left(\mathbf{1}-I_{\nu} P_{v}\right) \pi\left(f_{2}\right) \pi(w) \mathbf{1}_{\nu}\right\rangle_{\mathfrak{H}} \\
& =\left\langle\pi\left(f_{1}\right) \pi(w) \mathbf{1}_{\nu}, \pi\left(f_{2}\right) \pi(w) \mathbf{1}_{v}\right\rangle_{\mathfrak{H}}-\left\langle I_{v} P_{\nu} \pi\left(f_{1}\right) \pi(w) \mathbf{1}_{\nu}, I_{\nu} P_{\nu} \pi\left(f_{2}\right) \pi(w) \mathbf{1}_{v}\right\rangle_{\mathfrak{H}} \\
& =\left\langle\pi(w) \pi\left(w^{*}\left(f_{1}\right)\right) \mathbf{1}_{v}, \pi(w) \pi\left(w^{*}\left(f_{2}\right)\right) \mathbf{1}_{\nu}\right\rangle_{\mathfrak{H}}-\left\langle f_{1} \cdot P_{\nu} \pi(w) \mathbf{1}_{v}, f_{2} \cdot P_{v} \pi(w) \mathbf{1}_{v}\right\rangle_{\mu_{v}} \\
& =\left\langle w^{*}\left(f_{1}\right), w^{*}\left(f_{2}\right)\right\rangle_{\mu_{v}}-|c|^{2}\left\langle f_{1}, f_{2}\right\rangle_{\mu_{v}} \\
& =\left(1-|c|^{2}\right)\left\langle f_{1}, f_{2}\right\rangle_{\mu_{v}} \text {, }
\end{aligned}
$$

where $c$ is given by $P_{v} \pi(w) \mathbf{1}_{v}=c \mathbf{1}$. By the arguments above, $\left\|\psi_{0}\right\|^{2}=1-|c|^{2}$, implying

$$
\left\langle\pi\left(f_{1}\right) \psi, \pi\left(f_{2}\right) \psi\right\rangle_{\mathfrak{H}}=\left\langle f_{1}, f_{2}\right\rangle_{\mu_{v}}=\left\langle\pi\left(f_{1}\right) \mathbf{1}_{v}, \pi\left(f_{2}\right) \mathbf{1}_{\nu}\right\rangle_{\mathfrak{H}}
$$

for all $f_{1}, f_{2} \in C(X)$. The orthogonality of $\mathfrak{H}_{v}$ and $\mathfrak{H}_{\psi}$ follows directly from that of $\mathbf{1}_{v}$ and $\psi$. 
Definition 2.5. $\pi^{\prime}$ is called $\mathcal{W}^{\prime}$-natural iff, for any first-step decomposition $\bigoplus_{\nu \in \mathrm{N}} \pi_{\mu_{\nu}}$ and for all $\nu_{1}, \nu_{2} \in \mathrm{N}$ with $\mu_{\nu_{1}}=\mu_{\nu_{2}}$, an appearing $\pi^{\prime}\left(\mathcal{W}^{\prime}\right)$-invariance of $\mathfrak{H}_{v_{1}}$ implies that of $\mathfrak{H}_{\nu_{2}}$ and

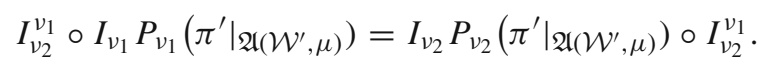

Obviously, $\pi^{\prime}$ is $\mathcal{W}^{\prime}$-natural, if the respective first-step decomposition is short. Moreover, if $\pi^{\prime}$ is $\mathcal{W}^{\prime}$-natural and if $\mu_{v_{1}}=\mu_{v_{2}}$, then $\mathbf{1}_{v_{1}}$ is $\pi^{\prime}\left(w^{\prime}\right)$-invariant iff $\mathbf{1}_{\nu_{2}}$ is $\pi^{\prime}\left(w^{\prime}\right)$ invariant.

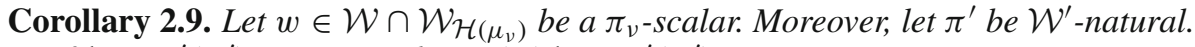

If $\mathbf{1}_{v}$ is $\pi^{\prime}\left(w^{\prime}\right)$-invariant, then $\pi(w) \mathbf{1}_{v}$ is $\pi^{\prime}\left(w^{\prime}\right)$-invariant.

Proof. Since $w^{\prime}$ is a $\pi_{v}$-unit, $\mathfrak{H}_{v}$ is $\pi^{\prime}\left(w^{\prime}\right)$-invariant. Hence,

$$
\pi^{\prime}\left(w^{\prime}\right) I_{\nu} P_{\nu} \pi(w) \mathbf{1}_{v}=\pi^{\prime}\left(w^{\prime}\right) c \mathbf{1}_{v}=c \mathbf{1}_{v}=I_{\nu} P_{\nu} \pi(w) \mathbf{1}_{v} .
$$

If $\psi_{0}:=\left(\mathbf{1}-I_{\nu} P_{v}\right) \pi(w) \mathbf{1}_{v}=0$, the statement is trivial. Otherwise, we know from the lemma above and the notations there that $\mathfrak{H}_{\nu}$ and $\mathfrak{H}_{\psi}$ are orthogonal. Choose a first-step decomposition of $\pi$ containing the representation $\pi_{\mu_{\nu}}$ for $\mathfrak{H}_{\nu}$ and for $\mathfrak{H}_{\psi}$. In fact, simply construct a first-step decomposition of the orthogonal complement of $\mathfrak{H}_{\nu} \oplus \mathfrak{H}_{\psi}$ in $\mathfrak{H}$. Now, since $\pi^{\prime}$ is $\mathcal{W}^{\prime}$-natural, $\psi$ is $\pi^{\prime}\left(w^{\prime}\right)$-invariant as well, by the $\pi^{\prime}\left(w^{\prime}\right)$-invariance of $\mathbf{1}_{v}$ and the lemma above. The proof follows from $\pi(w) \mathbf{1}_{v}=\psi_{0}+I_{v} P_{v} \pi(w) \mathbf{1}_{v}$.

Corollary 2.10. Let $w \in \mathcal{W} \cap \mathcal{W}_{\mathcal{H}\left(\mu_{v}\right)}$. Additionally, let $\pi^{\prime}$ be $\mathcal{W}^{\prime}$-natural, and let $\mathbf{1}_{v}$ be $\pi^{\prime}\left(w^{\prime}\right)$-invariant for some $w^{\prime} \in \mathcal{W}^{\prime}$.

If $w$ is a $\pi_{v}$-scalar, then $\pi\left(w^{\prime}(w)\right) \mathbf{1}_{v}=\pi^{\prime}\left(w^{\prime}\right) \pi(w) \mathbf{1}_{v}=\pi(w) \mathbf{1}_{v}$.

This means, in particular,

$w$ is a $\pi_{v}$-scalar. $\Longleftrightarrow w^{\prime}(w)$ is a $\pi_{v}$-scalar.

w is a $\pi_{v}$-unit. $\quad \Longleftrightarrow w^{\prime}(w)$ is a $\pi_{v}$-unit.

Corollary 2.11. Assume that $\pi^{\prime}$ is $\mathcal{W}^{\prime}$-natural and that $\mathbf{1}_{v}$ is $\pi^{\prime}\left(\mathcal{W}^{\prime}\right)$-invariant.

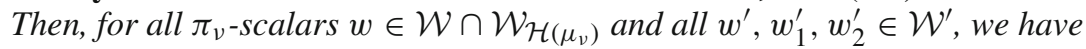

$$
\begin{aligned}
w^{\prime}(w) & =w_{1}^{\prime}(w) \circ w_{2}^{\prime}(w) \\
w^{\prime}(w)=w^{*} & \Longrightarrow w^{2} \text { is a } \text { is } \pi_{v} \text {-unit. }
\end{aligned}
$$

Proof. Using Corollary 2.9 we have in the first case,

$$
\begin{aligned}
\pi(w) \mathbf{1}_{v} & =\pi^{\prime}\left(w_{1}^{\prime}\right)^{*} \pi(w) \mathbf{1}_{v}=\pi^{\prime}\left(w_{1}^{\prime}\right)^{*} \pi\left(w^{\prime}(w)\right) \mathbf{1}_{v} \\
& =\pi^{\prime}\left(w_{1}^{\prime}\right)^{*} \pi\left(w_{1}^{\prime}(w) \circ w_{2}^{\prime}(w)\right) \mathbf{1}_{v}=\pi^{\prime}\left(w_{1}^{\prime}\right)^{*} \pi\left(w_{1}^{\prime}(w)\right) \pi\left(w_{2}^{\prime}(w)\right) \mathbf{1}_{v} \\
& =\pi(w) \pi^{\prime}\left(w_{1}^{\prime}\right)^{*} \pi^{\prime}\left(w_{2}^{\prime}\right) \pi(w) \pi^{\prime}\left(w_{2}^{\prime}\right)^{*} \mathbf{1}_{v}=\pi(w) \pi(w) \mathbf{1}_{v}
\end{aligned}
$$

and, in the second one,

$$
\pi(w) \mathbf{1}_{v}=\pi\left(w^{\prime}(w)\right) \mathbf{1}_{v}=\pi\left(w^{*}\right) \mathbf{1}_{v} .
$$


Lemma 2.12. If $w$ is a $\pi_{v}$-unit and $\mu_{v}$ equals $\mu$, then $P_{\nu} \pi(w)=\pi_{0}(w) P_{\nu}$.

Proof. We have

$$
\pi(w) f \mathbf{1}_{v}=\pi(w) \pi(f) \mathbf{1}_{v}=\pi(w(f)) \pi(w) \mathbf{1}_{v}=\pi(w(f)) \mathbf{1}_{v}=w(f) \mathbf{1}_{v},
$$

hence $P_{v} \pi(w) f \mathbf{1}_{v}=\pi_{0}(w) P_{v} f \mathbf{1}_{v}$ for all $f \in C(X)$. By continuity of $\pi_{0}(w)$ on $L_{2}(X, \mu)$ and by cyclicity of $\mathbf{1}_{v}$ w.r.t. $C(X)$, we get $P_{v} \pi(w)=\pi_{0}(w) P_{\nu}$ on $\mathfrak{H}_{v}$. Finally, both $P_{v} \pi(w)$ (by Lemma 2.6) and $\pi_{0}(w) P_{v}$ are zero on $\mathfrak{H}_{v}^{\perp}$.

Corollary 2.13. Let $w$ and $w_{0}$ be commuting elements in $\mathcal{W}$. Moreover, let $\mu_{v}=\mu$. If $w_{0}$ is a $\pi_{v}$-unit, then it leaves $P_{v} \pi(w) \mathbf{1}_{v}$ invariant.

Proof.

$$
\begin{aligned}
w_{0}\left(P_{\nu} \pi(w) \mathbf{1}_{\nu}\right) & \equiv \pi_{0}\left(w_{0}\right)\left(P_{\nu} \pi(w) \mathbf{1}_{\nu}\right) \\
& =P_{\nu} \pi\left(w_{0}\right) \pi(w) \mathbf{1}_{\nu} \quad(\text { Lemma 2.12) } \\
& =P_{\nu} \pi(w) \pi\left(w_{0}\right) \mathbf{1}_{v} \\
& =P_{\nu} \pi(w) \mathbf{1}_{v}
\end{aligned}
$$

2.3. Continuous $\mu_{0}$-generating systems. Until the end of this subsection, let $\mu_{0}$ be some measure on $X$.

Definition 2.6. A subset $\mathbf{E}$ of $C(X)$ is called continuous $\mu_{0}$-generating system iff

- $\mathbf{1} \in \mathbf{E}$ is orthogonal in $L_{2}\left(X, \mu_{0}\right)$ to each other element in $\mathbf{E}$ and

- $\operatorname{span}_{\mathbb{C}} \mathbf{E}$ is dense both in $C(X)$ and in $L_{2}\left(X, \mu_{0}\right)$.

Lemma 2.14. Let $\mathbf{E} \subseteq C(X)$ be a continuous $\mu_{0}$-generating system for some measure $\mu_{0}$, and let $\psi$ be a vector in $L_{2}\left(X, \mu_{0}\right)$.

Then $\langle f, \psi\rangle_{\mu_{0}}=0$ for all $\mathbf{1} \neq f \in \mathbf{E}$ implies that $\psi=c\|\psi\| \mathbf{1}$ for some $c \in U(1)$.

Proof. Use $L_{2}\left(X, \mu_{0}\right)=\overline{\operatorname{span}_{\mathbb{C}} \mathbf{E}}=\overline{\operatorname{span}_{\mathbb{C}}\{\mathbf{1}\} \oplus \operatorname{span}_{\mathbb{C}}(\mathbf{E}\{\mathbf{1}\})}=\mathbb{C} \mathbf{1} \oplus \overline{\operatorname{span}_{\mathbb{C}}(\mathbf{E}\{\mathbf{1}\})}$.

Lemma 2.15. If $\mathbf{E} \subseteq C(X)$ is a continuous generating system w.r.t. two measures $\mu_{1}$ and $\mu_{2}$, then $\mu_{1}$ equals $\mu_{2}$.

Proof. We have $\int_{X} f \mathrm{~d} \mu_{1}=\langle\mathbf{1}, f\rangle_{\mu_{1}}=0=\langle\mathbf{1}, f\rangle_{\mu_{2}}=\int_{X} f \mathrm{~d} \mu_{2}$ for all $\mathbf{1} \neq f \in \mathbf{E}$. Since $\operatorname{span}_{\mathbb{C}} \mathbf{E}$ is dense in $C(X)$, the assertion follows from the regularity of the measures.

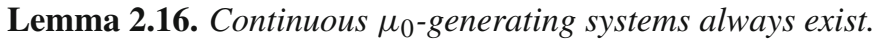

Proof. $C(X)$ always spans a dense subset in $L_{2}\left(X, \mu_{0}\right)$. Let now $\mathbf{E}$ contain $\mathbf{1}$ and all $f-\langle\mathbf{1}, f\rangle_{\mu_{0}} \mathbf{1}$ with $f$ in $C(X)$.

Lemma 2.17. Let $w \in \mathcal{W}$ be some element. Assume that $\pi^{\prime}$ is $\mathcal{W}^{\prime}$-natural and that $\mathbf{1}_{v}$ is $\mathcal{W}^{\prime}$-invariant. Moreover, let $\mathbf{E}_{0} \subseteq C(X)$ be some subset, such that for every non-constant $f \in \mathbf{E}_{0}$ there are infinitely many elements $\left\{w_{l}^{\prime}\right\}$ in $\left\langle\mathcal{W}^{\prime}\right\rangle$ commuting with $w$, such that $\left\{w_{\iota}^{\prime}(f)\right\} \subseteq C(X)$ forms an orthonormal system in $L_{2}\left(X, \mu_{v}\right)$.

Then $P_{v} \pi(w) \mathbf{1}_{v^{\prime}}$ is orthogonal to the span of $\mathbf{E}_{0}$ for all $v^{\prime} \in \mathrm{N}$ with $\mu_{v^{\prime}}=\mu_{v}$. Here, $\mathbf{E}_{0}$ is seen as a subset in $\mathfrak{H}_{v}$. 
Proof. Let $f \in \mathbf{E}_{0}$. Then there are infinitely many $\left\{w_{\iota}^{\prime}\right\}$ in $\left\langle\mathcal{W}^{\prime}\right\rangle$ commuting with $w$ and fulfilling

$$
\left\langle\pi\left(w_{\iota_{1}}^{\prime}(f)\right) \mathbf{1}_{v}, \pi\left(w_{\iota_{2}}^{\prime}(f)\right) \mathbf{1}_{\nu}\right\rangle_{\mathfrak{H}}=\left\langle w_{\iota_{1}}^{\prime}(f), w_{\iota_{2}}^{\prime}(f)\right\rangle_{\mu_{v}}=\delta_{\iota_{1} \iota_{2}} .
$$

By naturality, $\mathbf{1}_{v^{\prime}}$ is $\mathcal{W}^{\prime}$-invariant as well. Hence,

$$
\begin{aligned}
\left\langle\pi\left(w_{\iota}^{\prime}(f)\right) \mathbf{1}_{v}, \pi(w) \mathbf{1}_{v^{\prime}}\right\rangle_{\mathfrak{H}} & =\left\langle\pi^{\prime}\left(w_{\iota}^{\prime}\right) \pi(f) \pi^{\prime}\left(w_{\iota}^{\prime}\right)^{*} \mathbf{1}_{v}, \pi(w) \mathbf{1}_{v^{\prime}}\right\rangle_{\mathfrak{H}} \\
& =\left\langle\pi(f) \mathbf{1}_{v}, \pi^{\prime}\left(w_{\iota}^{\prime}\right)^{*} \pi(w) \mathbf{1}_{v^{\prime}}\right\rangle_{\mathfrak{H}} \\
& =\left\langle\pi(f) \mathbf{1}_{v}, \pi(w) \pi^{\prime}\left(w_{\iota}^{\prime}\right)^{*} \mathbf{1}_{v^{\prime}}\right\rangle_{\mathfrak{H}} \\
& =\left\langle\pi(f) \mathbf{1}_{v}, \pi(w) \mathbf{1}_{v^{\prime}}\right\rangle_{\mathfrak{H}}
\end{aligned}
$$

for all $\iota$. Consequently,

$$
\left\langle f, P_{v} \pi(w) \mathbf{1}_{v^{\prime}}\right\rangle_{\mu_{v}}=\left\langle\pi(f) \mathbf{1}_{v}, \pi(w) \mathbf{1}_{v^{\prime}}\right\rangle_{\mathfrak{H}}=0 .
$$

\subsection{Regularity.}

Definition 2.7. Precisely the elements of $\operatorname{Hom}(\mathbb{R}, \mathcal{W})$ are called one-parameter subgroups in $\mathcal{W}$, those in $\mathcal{R} \subseteq \operatorname{Hom}(\mathbb{R}, \mathcal{W})$ one-parameter $\mathcal{R}$-subgroups in $\mathcal{W}$.

Definition 2.8. A one-parameter subgroup is called regular iff it is weakly continuous.

Definition 2.9. A representation $\pi$ of $\mathfrak{A}(\mathcal{W}, \mu)$ is called regular w.r.t. $\mathcal{R}$ iff $\pi$ maps regular one-parameter $\mathcal{R}$-subgroups in $\mathcal{W}$ to weakly continuous one-parameter subgroups in $\pi(\mathcal{W})$.

If $\mathcal{R}$ is clear from the context, we will simply speak about regular representations.

Definition 2.10. $\bullet \quad$ Two one-parameter subgroups $t \longmapsto w_{1, t}$ and $t \longmapsto w_{2, t}$ in $\mathcal{W}$ are called commuting iff $w_{1, t_{1}}$ and $w_{2, t_{2}}$ commute for all $t_{1}, t_{2} \in \mathbb{R}$.

- The set given by all finite (pointwise) products of mutually commuting one-parameter $\mathcal{R}$-subgroups in $\mathcal{W}$ is denoted by $\langle\mathcal{R}\rangle$.

Lemma 2.18. The product of finitely many, mutually commuting one-parameter $\mathcal{R}$-subgroups in $\mathcal{W}$ is a one-parameter $\langle\mathcal{R}\rangle$-subgroup in $\langle\mathcal{W}\rangle$. Moreover, if $\pi$ is regular w.r.t. $\mathcal{R}$, then $\pi$ is regular w.r.t. $\langle\mathcal{R}\rangle$.

Proof. The first part is clear. For the second one use $\left\|\pi\left(w_{t}\right)\right\|_{\mathcal{B}(\mathfrak{H})} \leq\left\|w_{t}\right\|_{\mathfrak{A}(\mathcal{W}, \mu)}=1$ for all $t$ to show

$$
\begin{aligned}
\left\|\left(\prod_{i} \pi\left(w_{i, t}\right)\right) \psi-\psi\right\| & \leq \sum_{j}\left\|\left(\prod_{i<j} \pi\left(w_{i, t}\right)\right)\left(\pi\left(w_{j, t}\right) \psi-\psi\right)\right\| \\
& \leq \sum_{j}\left\|\pi\left(w_{j, t}\right) \psi-\psi\right\| \\
& \rightarrow 0
\end{aligned}
$$

for $t \rightarrow 0$.

Therefore, in what follows, we will often assume that $\mathcal{R}$ is replaced tacitly by $\langle\mathcal{R}\rangle$. 


\subsection{Splitting.}

Lemma 2.19. We have $\left\|\pi\left(w^{\prime}(f)\right)\right\|_{\mathcal{B}(\mathfrak{H})} \leq\|f\|_{\infty}$ for all $w^{\prime} \in \mathcal{W}_{\mathcal{H}(\mu)}$ and all $f \in$ $C(X)$.

Here, the equality holds if $\pi$ is faithful.

Proof. We get $\left\|\pi\left(w^{\prime}(f)\right)\right\|_{\mathcal{B}(\mathfrak{H})} \leq\left\|w^{\prime}(f)\right\|_{\mathfrak{A}(\mathcal{W}, \mu)}=\left\|w^{\prime}(f)\right\|_{\infty}=\|f\|_{\infty}$, since $w^{\prime}$ is a pull-back of a homeomorphism. If $\pi$ is faithful, then even $\left\|\pi\left(w^{\prime}(f)\right)\right\|_{\mathcal{B}(\mathfrak{H})}=$ $\left\|w^{\prime}(f)\right\|_{\mathfrak{A}(\mathcal{W}, \mu)}$.

Lemma 2.20. We have

$$
\sup _{w^{\prime} \in \mathcal{W}_{\mathcal{H}(\mu)}}\left|\left\langle\psi, \pi\left((w-\mathbf{1})\left(w^{\prime}(f)\right)\right) \psi\right\rangle_{\mathfrak{H}}\right| \leq 2\|f\|_{\infty}\|\psi\|_{\mathfrak{H}}\|(\pi(w)-\mathbf{1}) \psi\|_{\mathfrak{H}}
$$

for all $\psi \in \mathfrak{H}, w \in \mathcal{W}$ and $f \in C(X)$.

Proof. We have

$$
\begin{aligned}
& \left|\langle\psi, \pi((w-\mathbf{1})(f)) \psi\rangle_{\mathfrak{H}}\right| \\
& \quad=\left|\langle\psi, \pi(w(f)) \psi\rangle_{\mathfrak{H}}-\langle\psi, \pi(f) \psi\rangle_{\mathfrak{H}}\right| \\
& \quad=\left|\left\langle\psi, \pi(w) \pi(f) \pi(w)^{*} \psi\right\rangle_{\mathfrak{H}}-\langle\psi, \pi(f) \psi\rangle_{\mathfrak{H}}\right| \\
& \quad=\left|\left\langle\pi(w)^{*} \psi, \pi(f) \pi(w)^{*} \psi\right\rangle_{\mathfrak{H}}-\langle\psi, \pi(f) \psi\rangle_{\mathfrak{H}}\right| \\
& \quad \leq\left|\left\langle\left(\pi(w)^{*}-\mathbf{1}\right) \psi, \pi(f) \pi(w)^{*} \psi\right\rangle_{\mathfrak{H}}\right|+\left|\left\langle\psi, \pi(f)\left(\pi(w)^{*}-\mathbf{1}\right) \psi\right\rangle_{\mathfrak{H}}\right| \\
& \quad \leq\|\pi(f)\|_{\mathcal{B}(\mathfrak{H})}\left(\left\|\pi(w)^{*}\right\|_{\mathcal{B}(\mathfrak{H})}+1\right)\|\psi\|_{\mathfrak{H}}\left\|\left(\pi(w)^{*}-\mathbf{1}\right) \psi\right\|_{\mathfrak{H}} \\
& \quad \leq 2\|\pi(f)\|_{\mathcal{B}(\mathfrak{H})}\|\psi\|_{\mathfrak{H}}\|(\pi(w)-\mathbf{1}) \psi\|_{\mathfrak{H}}, \quad(\text { Unitarity of } w)
\end{aligned}
$$

hence, for all $w^{\prime} \in \mathcal{W}_{\mathcal{H}(\mu)}$,

$$
\begin{aligned}
\left|\left\langle\psi, \pi\left((w-\mathbf{1})\left(w^{\prime}(f)\right)\right) \psi\right\rangle_{\mathfrak{H}}\right| & \leq 2\left\|\pi\left(w^{\prime}(f)\right)\right\|_{\mathcal{B}(\mathfrak{H})}\|\psi\|_{\mathfrak{H}}\|(\pi(w)-\mathbf{1}) \psi\|_{\mathfrak{H}} \\
& \leq 2\|f\|_{\infty}\|\psi\|_{\mathfrak{H}}\|(\pi(w)-\mathbf{1}) \psi\|_{\mathfrak{H}}
\end{aligned}
$$

with Lemma 2.19 .

Definition 2.11. Let $\psi \in \mathfrak{H}$ be some vector.

- Let $f \in C(X)$.

We say $\mathcal{W}^{\prime}$ splits $\mathcal{W}$ at $\psi$ for $f$ iff there is a one-parameter $\mathcal{R}$-subgroup $w_{t}$ in $\mathcal{W}$, some $\varepsilon>0$ and some $t_{0}>0$, such that

$$
\sup _{w^{\prime} \in \mathcal{W}^{\prime}}\left|\left\langle\psi, \pi\left(\left(w_{t}-\mathbf{1}\right)\left(w^{\prime}(f)\right)\right) \psi\right\rangle_{\mathfrak{H}}\right| \geq \varepsilon
$$

for all $0 \neq|t|<t_{0}$.

- We say $\mathcal{W}^{\prime}$ splits $\mathcal{W}$ at $\psi$ iff there is a continuous $\mu$-generating system $\mathbf{E}$, such that $\mathcal{W}^{\prime}$ splits $\mathcal{W}$ at $\psi$ for every $f \in \mathbf{E}$ with $f \neq \mathbf{1}$ and $\langle\psi, \pi(f) \psi\rangle_{\mathfrak{H}} \neq 0$.

In other words, $w_{t}$ is not uniformly weakly continuous on the $\mathcal{W}^{\prime}$-span of $f$. Moreover, note that the splitting property actually refers to the choice of $\mathcal{R}$. Since, in general, we will have fixed $\mathcal{R}$, we drop this notion here.

Proposition 2.21. Assume that $\left.\pi \equiv \pi^{\prime}\right|_{\mathfrak{A}(\mathcal{W}, \mu)}$ is regular (w.r.t. $\left.\mathcal{R}\right)$.

If $\mathcal{W}^{\prime}$ splits $\mathcal{W}$ at $\mathbf{1}_{v_{0}}$, then $\mu_{\nu_{0}}$ equals $\mu$. 
Proof. Choose a continuous $\mu$-generating system $\mathbf{E}$, such that $\mathcal{W}^{\prime}$ splits $\mathcal{W}$ at $\mathbf{1}_{v_{0}}$ for every non-constant $f \in \mathbf{E}$ with $\langle\mathbf{1}, f\rangle_{\nu_{0}} \equiv\left\langle\mathbf{1}_{v_{0}}, \pi(f) \mathbf{1}_{v_{0}}\right\rangle_{\mathfrak{H}} \neq 0$. Assume there is such an $f$ with $\langle\mathbf{1}, f\rangle_{\nu_{0}} \neq 0$. Choose a one-parameter $\mathcal{R}$-subgroup $w_{t}$ in $\mathcal{W}$, some sufficiently small $\varepsilon>0$ and some $t_{0}>0$, such that

$$
\sup _{w^{\prime} \in \mathcal{W}^{\prime}}\left|\left\langle\mathbf{1}_{v_{0}}, \pi\left(\left(w_{t}-\mathbf{1}\right)\left(w^{\prime}(f)\right)\right) \mathbf{1}_{v_{0}}\right\rangle_{\mathfrak{H}}\right| \geq \varepsilon
$$

for all non-zero $|t|<t_{0}$. Hence, using Lemma 2.20,

$$
2\|f\|_{\infty}\left\|\pi\left(w_{t}\right) \mathbf{1}_{v_{0}}-\mathbf{1}_{v_{0}}\right\|_{\mathfrak{H}} \geq \sup _{w^{\prime} \in \mathcal{W}^{\prime}}\left|\left\langle\mathbf{1}_{v_{0}}, \pi\left(\left(w_{t}-\mathbf{1}\right)\left(w^{\prime}(f)\right)\right) \mathbf{1}_{v_{0}}\right\rangle_{\mathfrak{H}}\right| \geq \varepsilon
$$

for all non-zero $|t|<t_{0}$. This, however, is a contradiction to our assumption that $\pi$ is regular, i.e., $t \longmapsto \pi\left(w_{t}\right)$ is weakly continuous. Hence, $\langle\mathbf{1}, f\rangle_{\nu_{0}}=0$ for all $f$ in $\mathbf{E}$. By Lemma 2.15, we have $\mu=\mu_{\nu_{0}}$.

\section{6. $\Lambda$-Regularity.}

Definition 2.12. Let $A$ be any set.

- A set $\Lambda$ is called set of $A$-functions iff its elements are A-valued functions (i.e., there is no restriction for the domains of these functions).

- A set $\Lambda$ of A-functions is called topological (sequential) iff the domain of each $\lambda \in \Lambda$ is a topological (sequential topological) space.

Definition 2.13. Let $A$ be some subset of a $C^{*}$-algebra $\mathfrak{A}$, and let $\pi$ be a representation of $\mathfrak{A}$ on some Hilbert space $\mathfrak{H}$. Moreover, let $\Lambda$ be a set of topological A-functions.

Then $\pi$ is called $\Lambda$-regular iff the mapping

$$
\left\langle\psi_{1}, \pi(\lambda(\cdot)) \psi_{2}\right\rangle_{\mathfrak{H}}: \operatorname{dom} \lambda \longrightarrow \mathbb{C}
$$

is continuous for all $\psi_{1}, \psi_{2} \in \mathfrak{H}$ and each $\lambda \in \Lambda$.

Remark. The ordinary regularity uses $\operatorname{dom} \lambda=\mathbb{R}$, where $\lambda: t \longmapsto w_{t}$ runs over all one-parameter $\mathcal{R}$-subgroups.

Let us return to the case that $\pi$ is a representation of $\mathfrak{A}(\mathcal{W}, \mu)$ on $\mathfrak{H}$.

Proposition 2.22. Let $\pi$ be $\Lambda$-regularfor some set $\Lambda$ of $\mathcal{W}$-functions. Fixfor each $\lambda \in \Lambda$ some subset $Y_{\lambda}$ in dom $\lambda$, such that $\lambda\left(Y_{\lambda}\right)$ consists of $\pi_{\nu}$-units only and $\bigcup_{\lambda \in \Lambda} \lambda\left(\overline{Y_{\lambda}}\right)$ generates $\mathcal{W}$.

Then every $w \in \mathcal{W}$ is a $\pi_{v}$-unit.

Proof. For all $\lambda \in \Lambda$ and all $y \in Y_{\lambda}$, we have

$$
\left\langle\mathbf{1}_{v}, \pi(\lambda(y)) \mathbf{1}_{v}\right\rangle_{\mathfrak{H}}=\left\langle\mathbf{1}_{v}, \mathbf{1}_{v}\right\rangle_{\mathfrak{H}}=1 .
$$

Consequently, by $\Lambda$-regularity, we even have $\left\langle\mathbf{1}_{v}, \pi(\lambda(y)) \mathbf{1}_{\nu}\right\rangle_{\mathfrak{H}}=1$ for all $y \in \overline{Y_{\lambda}}$, hence $\pi(\lambda(y)) \mathbf{1}_{v}=\mathbf{1}_{v}$, i.e., $\lambda\left(\overline{Y_{\lambda}}\right)$ contains $\pi_{v}$-units only. Since these sets generate full $\mathcal{W}$ and since, obviously, products and inverses of $\pi_{v}$-units are $\pi_{v}$-units again, all elements of $\mathcal{W}$ are $\pi_{v}$-units. 


\section{Quantum Geometric Background}

3.1. Quantum geometric Hilbert space. In the remaining sections we will apply the general framework of Sect. 2 to quantum geometry. First, however, let us briefly recall in this subsection the basic facts and notations needed in the following. General expositions can be found in $[6,4,3]$ for the analytic framework. The smooth case is dealt with in $[8,7,27]$. The facts on hyphs and the conventions are due to $[14,16,19]$.

Let $\mathbf{G}$ be some arbitrary connected compact Lie group and $M$ be some manifold. We let $M$ be equipped with an arbitrary, but fixed differential structure. Later, we will restrict ourselves to analytic (or, if so desired, semianalytic) manifolds. A path is a piecewise differentiable map from $[0,1]$ to $M$, whereas differentiability is always understood in the chosen smoothness class. Moreover, we may restrict ourselves to use piecewise embedded paths only. A path is trivial iff its image is a single point. Two paths $\gamma_{1}$ and $\gamma_{2}$ are composable iff the end point $\gamma_{1}(1)$ of the first one coincides with the starting point $\gamma_{2}(0)$ of the second one. If they are composable, their product is given by

$$
\left(\gamma_{1} \gamma_{2}\right)(t):=\left\{\begin{array}{ll}
\gamma_{1}(2 t) & \text { for } t \in\left[0, \frac{1}{2}\right] \\
\gamma_{2}(2 t-1) & \text { for } t \in\left[\frac{1}{2}, 1\right]
\end{array} .\right.
$$

An edge $e$ is a path having no self-intersections, i.e., $e\left(t_{1}\right)=e\left(t_{2}\right)$ implies that $\left|t_{1}-t_{2}\right|$ either equals 0 or 1 . Two paths $\gamma_{1}$ and $\gamma_{2}$ coincide up to the parametrization iff there is some orientation preserving piecewise diffeomorphism $\phi:[0,1] \longrightarrow[0,1]$, such that $\gamma_{1}=\gamma_{2} \circ \phi$. A path is called finite iff it equals up to the parametrization a finite product of edges and trivial paths. In what follows, every path will be assumed to be finite. Next, two paths are equivalent iff there is a finite sequence of paths, such that two subsequent paths coincide up to the parametrization or up to insertion or deletion of retracings $\delta \delta^{-1}$. Finally, we denote the set of all paths by $\mathcal{P}_{\text {gen }}$, that of all equivalence classes of paths by $\mathcal{P}$. The multiplication of paths naturally turns $\mathcal{P}$ into a groupoid. Usually (but not in Subsects. 3.2 and 3.3), paths are understood to be equivalence classes of paths.

Initial and final segments of paths are naturally defined. We will write $\gamma_{1} \uparrow \uparrow \gamma_{2}$ iff there is some path $\gamma$ being (possibly up to the parametrization) an initial path of both $\gamma_{1}$ and $\gamma_{2}$. A hyph $v$ is some finite collection $\left(\gamma_{1}, \ldots, \gamma_{n}\right)$ of edges each having a "free" point. This means, for at least one direction none of the segments of $\gamma_{i}$ starting in that point in this direction, is a full segment of some of the $\gamma_{j}$ with $j<i$. Graphs and webs are special hyphs. The subgroupoid generated (freely) by the paths in a hyph $v$ will be denoted by $\mathcal{P}_{v}$. Hyphs are ordered in the natural way. In particular, $v^{\prime} \leq v^{\prime \prime}$ implies $\mathcal{P}_{v^{\prime}} \subseteq \mathcal{P}_{v^{\prime \prime}}$

The set $\overline{\mathcal{A}}$ of generalized connections $\bar{A}$ is now defined by

$$
\overline{\mathcal{A}}:=\lim _{v} \overline{\mathcal{A}}_{v} \cong \operatorname{Hom}(\mathcal{P}, \mathbf{G})
$$

with $\overline{\mathcal{A}}_{\boldsymbol{\gamma}}:=\operatorname{Hom}\left(\mathcal{P}_{\boldsymbol{\gamma}}, \mathbf{G}\right) \subseteq \mathbf{G}^{\# \boldsymbol{\gamma}}$ given the topology which is induced by that of $\mathbf{G}$, for all finite tuples $\gamma$ of paths. Moreover, we define the (always continuous) map $\pi_{\boldsymbol{\gamma}}: \overline{\mathcal{A}} \longrightarrow \mathbf{G}^{\# \boldsymbol{\gamma}}$ by $\pi_{\boldsymbol{\gamma}}(\bar{A}):=\bar{A}(\boldsymbol{\gamma}) \equiv h_{\bar{A}}(\boldsymbol{\gamma})$. Note that $\pi_{\boldsymbol{\gamma}}$ is surjective, if $\boldsymbol{\gamma}$ is a hyph. Finally, for compact $\mathbf{G}$, the Ashtekar-Lewandowski measure $\mu_{0}$ is the unique regular Borel measure on $\overline{\mathcal{A}}$ whose push-forward $\left(\pi_{v}\right)_{*} \mu_{0}$ to $\overline{\mathcal{A}}_{v} \cong \mathbf{G}^{\# v}$ coincides with the Haar measure there for every hyph $v$. It is used to span the auxiliary Hilbert space $\mathfrak{H}_{\text {aux }}:=L_{2}\left(\overline{\mathcal{A}}, \mu_{0}\right)$ of quantum geometry with scalar product $\langle\cdot, \cdot\rangle$. 
If we included (generalized) gauge transforms into our considerations and studied the analytic category only, we could use the spin-network states to get a basis of $\mathfrak{H}_{\text {aux, inv }}=$ $L_{2}\left(\overline{\mathcal{A}} / \overline{\mathcal{G}}, \mu_{0}\right)$ with $\overline{\mathcal{G}}$ being the group of generalized gauge transforms. Here, however, we want to include gauge-variant functions as well and, moreover, do not want to restrict the smoothness class at the beginning. Therefore, we will consider now generating systems for $\mathfrak{H}_{\text {aux }}$. For this, first of all, let us fix a representative in each equivalence class of irreducible representations of $\mathbf{G}$, which we will refer to below. When considering matrix indices for matrices on some Euclidean space $V$, we assume that the underlying vectors are normalized. This means that for all $A \in$ End $V$ we have $\left|A_{j}^{i}\right| \leq\|A\|$, where $\|\cdot\|$ denotes the standard operator norm.

Definition 3.1. • For each non-trivial irreducible representation of $\mathbf{G}$, we define $\mathcal{M}^{\phi}$ to be the set

$$
\mathcal{M}^{\phi}:=\bigcup_{m, n}\left\{\sqrt{\operatorname{dim} \phi} \phi_{n}^{m}\right\}
$$

of normalized matrix functions, where $m, n$ run over the set of matrix indices for $\phi$.

- We define $\mathcal{M}$ to be the set

$$
\mathcal{M}:=\bigcup_{\phi} \mathcal{M}^{\phi}=\bigcup_{\phi, m, n}\left\{\sqrt{\operatorname{dim} \phi} \phi_{n}^{m}\right\}
$$

of normalized matrix functions, where $\phi$ runs over the set of all (equivalence classes of) non-trivial irreducible representations of $\mathbf{G}$.

- For every hyph $v$ with edges $\gamma_{1}, \ldots, \gamma_{I}$ we define the set $\mathcal{M}_{v}$ of gauge-variant spin network states (gSN) of $v$ by

$$
\mathcal{M}_{v}:=\bigotimes_{i} \mathcal{M} \circ \pi_{\gamma_{i}}
$$

If $v$ is the empty hyph, we have $\mathcal{M}_{v}:=\{1\}$. The set of all gauge-variant spin network states will be denoted by $\mathcal{M}_{\mathrm{SN}}$.

- More compactly, we set $\left(T_{\phi, \gamma}\right)_{n}^{m}:=\sqrt{\operatorname{dim} \phi} \phi_{n}^{m} \circ \pi_{\gamma}$ and

$$
\left(T_{\boldsymbol{\phi}, \boldsymbol{\gamma}}\right)_{\boldsymbol{n}}^{\boldsymbol{m}}:=\sqrt{\operatorname{dim} \boldsymbol{\phi}} \boldsymbol{\phi}_{n}^{m} \circ \pi_{\boldsymbol{\gamma}} \equiv \bigotimes_{k} \sqrt{\operatorname{dim} \phi_{k}}\left(\phi_{k}\right)_{n_{k}}^{m_{k}} \circ \pi_{\gamma_{k}}
$$

Observe that we get the same gauge-variant spin network state again if we simultaneously revert the orientations of an arbitrary number of edges and dualize the corresponding representations. This trivial overcompleteness will be ignored in the following, i.e., we will always identify graphs and hyphs differing in the ordering or the orientation of the edges only.

Let us now recall

Lemma 3.1. For every hyph $v$, the set $\mathcal{M}_{v}$ of gauge-variant spin networks on $v$ is an orthonormal set in $L_{2}\left(\overrightarrow{\mathcal{A}}, \mu_{0}\right)$.

Note that (even after admitting only one edge orientation per hyph) $\bigcup_{v} \mathcal{M}_{v}$ is a generating system for, but not an orthonormal set in $L_{2}\left(\overline{\mathcal{A}}, \mu_{0}\right)$. This would still be the case, if we were in the (semi)analytic category and use graphs only (see below). In particular, we have 
Lemma 3.2. - We have $\mathcal{M}_{v^{\prime}} \subseteq \operatorname{span} \mathcal{M}_{v}$ for all $v \geq v^{\prime}$.

- We have $\mathcal{M}_{\gamma}^{\phi} \subseteq \operatorname{span} \mathcal{M}_{v}^{\phi}$ for all $v=\left\{\gamma_{1}, \ldots, \gamma_{n}\right\} \geq \gamma$ with $\prod_{i} \gamma_{i}=\gamma$. Here, $\mathcal{M}_{v}^{\phi}:=\bigotimes_{i} \mathcal{M}^{\phi} \circ \pi_{\gamma_{i}}$.

Lemma 3.3. $\mathcal{M}_{\mathrm{SN}}$ is a continuous $\mu$-generating system in $L_{2}\left(\overline{\mathcal{A}}, \mu_{0}\right)$.

Nevertheless, we will be looking for orthogonal decompositions of $L_{2}\left(\overline{\mathcal{A}}, \mu_{0}\right)$. For that purpose, we will have to single out orthogonal subsets of gauge-variant spin network functions: Until the end of this subsection we will now consider piecewise analytic paths only.

In contrast to the standard, i.e., gauge-invariant spin network states, the gauge-variant ones do not form an orthonormal basis for $L_{2}\left(\overline{\mathcal{A}}, \mu_{0}\right)$ even after dropping some subset of them. The problem are the states arising in the decomposition of an edge into a product of subedges, i.e., having two-valent vertices. In the gauge-invariant case they can be dropped since, by invariance, they reproduce the original state. Here, however, in the gauge-variant case, we get a sum like

$$
\left(T_{\gamma_{1} \gamma_{2}, \phi}\right)_{n}^{m}=\frac{1}{\sqrt{\operatorname{dim} \phi}} \sum_{r}\left(T_{\gamma_{1}, \phi}\right)_{r}^{m} \otimes\left(T_{\gamma_{2}, \phi}\right)_{n}^{r},
$$

where the $(\operatorname{dim} \phi)$ gauge-variant spin network states together with that at the left-hand side span a $(\operatorname{dim} \phi)$-dimensional subspace of $L_{2}\left(\overline{\mathcal{A}}, \mu_{0}\right)$. We might simply drop the one at the left-hand side, but this would lead to consistency troubles since we could want to decompose those at the right-hand side again. A possible solution for this dilemma is given by the extended spin network states as defined by Ashtekar and Lewandowski in [5]. We do not want to introduce that notion here, but only study the "most dangerous" cases in our framework - namely, those gSN with "matching" indices ${ }^{4}$ at each two-valent vertex. In the decomposition of the $\gamma_{1} \gamma_{2}$-state above, this concerns the vector at $\gamma_{1}(1)=\gamma_{2}(0)$.

Lemma 3.4. Let two gauge-variant spin networks states $T:=\left(T_{\boldsymbol{\phi}, \gamma}\right)_{n}^{\boldsymbol{m}}$ and $T^{\prime}:=$ $\left(T_{\boldsymbol{\phi}^{\prime}, \boldsymbol{\gamma}^{\prime}}\right)_{\boldsymbol{n}^{\prime}}^{\boldsymbol{m}^{\prime}}$ with graphs $\boldsymbol{\gamma}$ and $\boldsymbol{\gamma}^{\prime}$ be given.

Then $T$ and $T^{\prime}$ are orthogonal in $L_{2}\left(\overline{\mathcal{A}}, \mu_{0}\right)$ if

- $\operatorname{im} \boldsymbol{\gamma} \neq \operatorname{im} \boldsymbol{\gamma}^{\prime}$;

- there is a point $m \in$ int $\boldsymbol{\gamma} \cap$ int $\boldsymbol{\gamma}^{\prime}$, such that the representations for the edges in $\boldsymbol{\gamma}$ and $\boldsymbol{\gamma}^{\prime}$ running through $m$ do not coincide;

- there is some $m \in M$ being a two-valent vertex with non-matching indices for one and being interior for the other graph; or

- there is some $m \in M$ being a two-valent vertex for both graphs, whereas both "incoming" or both "outgoing" indices are different.

Note that matrix indices are regarded as different if they belong to different representations.

Proof. The first two cases are obvious. The third one is clear observing our example above. Namely, decompose one of the graphs, say $\boldsymbol{\gamma}^{\prime}$, by inserting $m$ as a vertex. In the decomposition of $T^{\prime}$ into a sum of gauge-variant spin network states of the enlarged

\footnotetext{
4 Recall that a gSN is said to have "matching" indices at a two-valent vertex $m$ iff the lower index, assigned to the incoming edge at $m$, and the upper index for the outgoing one are equal. Note that we possibly have to invert orientations before, in order to have an incoming and an outgoing edge at a two-valent vertex.
} 
graph, the indices of every addend are matching. By the orthogonality properties of matrix functions w.r.t. the Haar measure, we get the assertion. The last case is now clear as well.

Definition 3.2. Let $\gamma$ be an edge and $\phi$ be a non-trivial irreducible representation of $\mathbf{G}$ and let $T:=\left(T_{\boldsymbol{\phi}, \boldsymbol{\gamma}}\right)_{\boldsymbol{n}}^{\boldsymbol{m}}$ be a gauge-variant spin network state.

1. If $\gamma$ is non-closed, then $T$ is called $(\gamma, \phi)$-based iff

- $\gamma=\gamma_{1} \circ \cdots \circ \gamma_{\#} \gamma$;

- $\phi_{k}=\phi$ for all $k$; and

- all indices at two-valent vertices are matching, i.e., $m_{k+1}=n_{k}$ for all $k$.

2. If $\gamma$ is closed, then $T$ is called $(\gamma, \phi)$-based iff

- $\gamma_{1} \circ \cdots \circ \gamma_{\# \gamma}$ equals $\gamma$ or equals $\left.\left.\gamma\right|_{[\tau, 1]} \circ \gamma\right|_{[0, \tau]}$ for some $\tau \in(0,1)$;

- $\phi_{k}=\phi$ for all $k$; and

- all indices at two-valent vertices are matching, i.e., $m_{k+1}=n_{k}$ for all $k$ and $m_{1}=n_{\# \gamma}$.

The set of all $(\gamma, \phi)$-based gauge-variant spin network states will be denoted by $\mathcal{B}_{\gamma, \phi}$. Moreover, we set $\mathcal{B}_{\gamma}:=\{\mathbf{1}\} \cup \cup_{\phi} \mathcal{B}_{\gamma, \phi}$, where the union runs over all non-trivial irreducible representations of $\mathbf{G}$. It contains precisely the $\gamma$-based gauge-variant spin network states.

Note again that $T$ is $(\gamma, \phi)$-based if for some orientation and some ordering of $\gamma$, the conditions above are met.

Lemma 3.5. $\mathcal{B}_{\gamma, \phi}$ is orthogonal to its complement in the set of all gauge-variant spin network states, for every edge $\gamma$ and every irreducible representation $\phi$ of $\mathbf{G}$.

Proof. Let $T=\left(T_{\phi, \gamma}\right)_{n}^{\boldsymbol{m}}$ be a gSN not contained in $\mathcal{B}_{\gamma, \phi}$. If im $\gamma \neq \operatorname{im} \gamma$, the situation is clear. The same is true for $\phi_{k} \neq \phi$ for some $k$. Let now im $\gamma=\operatorname{im} \gamma$ and $\phi_{k}=\phi$ for all $k$. Then, possibly after modifying ordering or orientations, we have $\gamma=\gamma_{1} \cdots \gamma_{n}$. Moreover, every vertex of $\boldsymbol{\gamma}$ is at most two-valent. Thus, the proof follows from Lemma 3.4 .

Corollary 3.6. For every edge $\gamma$, the Hilbert space $L_{2}\left(\overline{\mathcal{A}}, \mu_{0}\right)$ is the closure of

$$
\left(\bigoplus_{\phi} \operatorname{span} \mathcal{B}_{\gamma, \phi}\right) \oplus \mathbb{C} \mathbf{1} \oplus \operatorname{span}\left(\mathcal{M}_{\mathrm{SN}} \mathcal{B}_{\gamma}\right)
$$

3.2. Decomposition of paths. In the following we will study the intersection behaviour between paths and (generalized) surfaces. For this, we first consider how paths can be decomposed. Most of the relevant definitions and assertions are given in [13]. We will quote where appropriate and will simplify some assumptions and, therefore, proofs. Note that in this subsection we will often distinguish between $\mathcal{P}$ and $\mathcal{P}_{\text {gen }}$; paths here are genuine maps from $[0,1]$ to $M$, not equivalence classes.

\subsubsection{Completeness.}

Definition 3.3. Let $\gamma$ be some path.

Then a finite sequence $\gamma:=\left(\gamma_{1}, \ldots, \gamma_{n}\right)$ in $\mathcal{P}_{\text {gen }}$ is called decomposition of $\gamma$ iff $\gamma_{1} \cdots \gamma_{n}$ equals $\gamma$ up to the parametrization. 
This definition is well defined, since $\gamma_{1}\left(\gamma_{2} \gamma_{3}\right)$ equals $\left(\gamma_{1} \gamma_{2}\right) \gamma_{3}$ up to the parametrization. Moreover, observe that every reparametrization of $\gamma$ gives a decomposition of $\gamma$.

If confusion is unlikely, we identify $\gamma_{1} \cdots \gamma_{n},\left\{\gamma_{1}, \ldots, \gamma_{n}\right\}$, and $\left(\gamma_{1}, \ldots, \gamma_{n}\right)$.

Definition 3.4. Let $\gamma:=\gamma_{1} \cdots \gamma_{I}$ and $\delta:=\delta_{1} \cdots \delta_{J}$ be decompositions of some path $\gamma$. Then $\boldsymbol{\gamma}$ is a refinement of $\delta$ iff there are $0=I_{0}<I_{1}<\cdots<I_{J}=I$, such that $\gamma_{I_{j-1}+1} \cdots \gamma_{I_{j}}$ is a decomposition of $\delta_{j}$ for all $j=1, \ldots, J$. We write ${ }^{5} \boldsymbol{\gamma} \geq \boldsymbol{\delta}$ iff $\boldsymbol{\gamma}$ is $a$ refinement of $\delta$.

It can easily be shown [13] that the set of all decompositions of a path $\gamma$ is directed w.r.t. $\geq$.

Definition 3.5. • A subset $\mathcal{Q}$ of $\mathcal{P}_{\text {gen }}$ is called hereditary iff for each $\gamma \in \mathcal{Q}$

1. the inverse of $\gamma$ is in $\mathcal{Q}$ again, and

2. every decomposition of $\gamma$ consists of paths in $\mathcal{Q}$.

- A subset $\mathcal{Q}$ of $\mathcal{P}_{\text {gen }}$ is called complete iff it is hereditary and every path in $\mathcal{P}_{\text {gen }}$ has a decomposition into paths in $\mathcal{Q}$.

A decomposition consisting of paths in $\mathcal{Q}$ only, will be called $\mathcal{Q}$-decomposition.

Lemma 3.7. Let $\mathcal{Q} \subseteq \mathcal{P}_{\text {gen }}$ be complete.

Then for every hyph $v$ there is a hyph $v^{\prime} \geq v$ with $v^{\prime} \subseteq \mathcal{Q}$.

Proof. First decompose each $\gamma \in v$ into paths in $\mathcal{Q}$. Collect all these paths in a set $\boldsymbol{\gamma}^{\prime} \geq v$. Since $\boldsymbol{\gamma}^{\prime}$ may be not a hyph again, refine, if necessary, the paths in $\boldsymbol{\gamma}^{\prime}$ further to get a hyph $v^{\prime} \geq \boldsymbol{\gamma}^{\prime} \geq v$ [14]. By completeness, $v^{\prime}$ contains only paths in $\mathcal{Q}$.

Lemma 3.8. The set of all edges and trivial paths in $\mathcal{P}_{\text {gen }}$ is complete.

\subsubsection{Main construction.}

Definition 3.6. Let $\mathcal{Q}$ be some hereditary subset of $\mathcal{P}_{\text {gen }}$.

Then a map $\rho: \mathcal{Q} \longrightarrow \mathbf{G}$ is called $\mathcal{Q}$-germ iff for all $\gamma \in \mathcal{Q}$

1. $\rho\left(\gamma^{-1}\right)=\rho(\gamma)^{-1}$, and

2. $\rho(\gamma)=\rho\left(\gamma_{1}\right) \rho\left(\gamma_{2}\right)$ for all decompositions $\gamma_{1} \gamma_{2}$ of $\gamma$.

The set of all $\mathcal{Q}$-germs from $\mathcal{Q}$ to $\mathbf{G}$ is denoted by $\operatorname{Germ}(\mathcal{Q}, \mathbf{G})$.

Observe that $\rho(\gamma)$ and $\rho(\delta)$ coincide if $\gamma$ and $\delta$ coincide up to the parametrization. In fact, since every decomposition $\gamma_{1} \gamma_{2}$ of $\gamma$ is also some for $\delta$, we may apply Property 2 above.

Note that we will shortly speak about germs instead of $\mathcal{Q}$-germs, provided the domain $\mathcal{Q}$ is clear from the context.

Proposition 3.9. Let $\mathcal{Q}$ be some complete subset of $\mathcal{P}_{\text {gen }}$, and let $\rho: \mathcal{Q} \longrightarrow \mathbf{G}$ be a germ.

Then we have:

- There is a unique germ $\widehat{\rho}: \mathcal{P}_{\text {gen }} \longrightarrow \mathbf{G}$ extending $\rho$.

\footnotetext{
5 By a little misuse of notation we denote both graphs and decompositions by $\boldsymbol{\gamma}, \boldsymbol{\delta}$, etc., and denote both the relation on the set of hyphs (or graphs) and that of refinement by $\geq$. Confusion should be unlikely.
} 
- The map $\widehat{\rho}$ is given by

$$
\widehat{\rho}(\gamma)=\prod_{i=1}^{I} \rho\left(\gamma_{i}\right)
$$

for each $\gamma \in \mathcal{P}_{\text {gen, }}$ where $\gamma_{1} \cdots \gamma_{n}$ is any ${ }^{6} \mathcal{Q}$-decomposition of $\gamma$.

- The map $\widehat{\rho}$ is constant on equivalence classes in $\mathcal{P}_{\text {gen }}$.

- The induced map $[\widehat{\rho}]: \mathcal{P} \longrightarrow \mathbf{G}$ is a homomorphism, i.e., it is an element of $\overline{\mathcal{A}}$.

Proof. Let us first define the desired map $\widehat{\rho}$ as given in the proposition above and now check its properties.

1. $\widehat{\rho}$ does not depend on the choice of the $\mathcal{Q}$-decomposition.

Let $\gamma$ and $\delta$ be two $\mathcal{Q}$-decompositions of $\gamma$. Since, by assumption, every path in $\mathcal{Q}$ has $\mathcal{Q}$-decompositions only, and since the set of decompositions of a path is directed w.r.t. $\geq$, we may assume $\boldsymbol{\gamma} \geq \boldsymbol{\delta}$. But, in this case the well-definedness follows directly from the definitions and germ property 2 of $\rho$.

2. $\widehat{\rho}$ is constant on equivalence classes in $\mathcal{P}_{\text {gen }}$.

Let $\gamma$ and $\delta$ in $\mathcal{P}_{\text {gen }}$ be equivalent. By definition, it is sufficient to check the following two cases:

- $\quad \gamma$ and $\delta$ coincide up to the parametrization.

This case is trivial, since every $\mathcal{Q}$-decomposition of $\gamma$ is also one of $\delta$. Hence, $\rho(\gamma)=\rho(\delta)$.

- There is some $\varepsilon$ in $\mathcal{P}_{\text {gen }}$ and some decomposition $\gamma_{1} \gamma_{2}$ of $\gamma$, such that $\delta$ equals the product of $\gamma_{1}, \varepsilon, \varepsilon^{-1}$ and $\gamma_{2}$.

Now, in this case, choose some $\mathcal{Q}$-decompositions $\varepsilon_{1} \cdots \varepsilon_{K}$ of $\varepsilon$ and $\gamma_{s} \cdots \gamma_{s I_{s}}$ of $\gamma_{s}$ with $s=1,2$. Then $\gamma_{11} \cdots \gamma_{1 I_{1}} \gamma_{21} \cdots \gamma_{2 I_{2}}$ is a $\mathcal{Q}$-decomposition of $\gamma$ and $\gamma_{11} \cdots \gamma_{1 I_{1}} \varepsilon_{1} \cdots \varepsilon_{K} \varepsilon_{K}^{-1} \cdots \varepsilon_{1}^{-1} \gamma_{21} \cdots \gamma_{2 I_{2}}$ one of $\delta$. Hence, we have

$$
\begin{aligned}
& \widehat{\rho}(\delta)=\rho\left(\gamma_{11}\right) \cdots \rho\left(\gamma_{1 I_{1}}\right) \rho\left(\varepsilon_{1}\right) \cdots \rho\left(\varepsilon_{K}\right) \\
& \rho\left(\varepsilon_{K}^{-1}\right) \cdots \rho\left(\varepsilon_{1}^{-1}\right) \rho\left(\gamma_{21}\right) \cdots \rho\left(\gamma_{2 I_{2}}\right) \quad \text { (Definition of } \widehat{\rho} \text { ) } \\
& =\rho\left(\gamma_{11}\right) \cdots \rho\left(\gamma_{1 I_{1}}\right) \rho\left(\gamma_{21}\right) \cdots \rho\left(\gamma_{2 I_{2}}\right) \quad \text { (Property } 1 \text { of } \rho \text { ) } \\
& =\widehat{\rho}(\gamma) . \quad \text { (Definition of } \widehat{\rho})
\end{aligned}
$$

3. $\widehat{\rho}$ is a germ extending $\rho$, and $[\widehat{\rho}]$ is a homomorphism.

This is proven as the statements above.

4. $\widehat{\rho}$ is the only germ extending $\rho$.

If $\widehat{\rho}^{\prime}$ is some other germ extending $\rho$ different from $\widehat{\rho}$, then there is some $\gamma \in \mathcal{P}_{\text {gen }}$ with $\widehat{\rho}^{\prime}(\gamma) \neq \widehat{\rho}(\gamma)$. Now, choose a $\mathcal{Q}$-decomposition $\gamma_{1} \cdots \gamma_{I}$ of $\gamma$. By the properties of a germ, there is some $i$ with $\widehat{\rho}^{\prime}\left(\gamma_{i}\right) \neq \widehat{\rho}\left(\gamma_{i}\right)$. However, since both $\widehat{\rho}^{\prime}$ and $\widehat{\rho}$ extend $\rho$, both sides are equal to $\rho\left(\gamma_{i}\right)$. Contradiction.

Proposition 3.10. Let $\mathcal{Q}$ be some complete subset of $\mathcal{P}_{\text {gen }}$. Let $X$ be some topological space, and let $\lambda: X \longrightarrow \operatorname{Germ}(\mathcal{Q}, \mathbf{G})$ be some map. Finally, assume that the map $(\lambda(\cdot))(\gamma): X \longrightarrow \mathbf{G}$ is continuous for all $\gamma \in \mathcal{Q}$.

Then

$$
\begin{aligned}
\Theta_{\lambda}: X & \longrightarrow \overline{\mathcal{A}} \\
x & \longmapsto[\overline{\lambda(x)}]
\end{aligned}
$$

is continuous, where $\widehat{r}$ is given as in Proposition 3.9 .

\footnotetext{
${ }^{6}$ Recall that, by completeness of $\mathcal{Q}$, such a decomposition exists always.
} 
Proof. It is sufficient [19] to prove that $\pi_{\gamma} \circ \Theta_{\lambda}: X \longrightarrow \mathbf{G}$ is continuous for all edges $\gamma$. Since the multiplication in $\mathbf{G}$ is continuous and $\mathcal{Q}$ is complete, we even may restrict ourselves to the cases of $\gamma \in \mathcal{Q}$. Here, however, the assertion follows immediately from

$$
\left.\left(\pi_{\gamma} \circ \Theta_{\lambda}\right)(x) \equiv \pi_{\gamma}([\widehat{\lambda(x)}])=\widehat{[\lambda(x)}\right]([\gamma])=\widehat{\lambda(x)}(\gamma) \equiv \lambda(x)(\gamma),
$$

i.e., $\pi_{\gamma} \circ \Theta_{\lambda}=(\lambda(\cdot))(\gamma)$ for all $\gamma \in \mathcal{Q}$.

Lemma 3.11. Two generalized connections coincide iff they coincide for all (equivalence classes of) paths of a complete subset of $\mathcal{P}_{\text {gen }}$.

\subsubsection{Application to Weyl-type operators.}

Definition 3.7. Let $\mathcal{Q}$ be some hereditary subset of $\mathcal{P}_{\text {gen }}$.

Then a $\operatorname{map} \kappa: \mathcal{Q} \longrightarrow \mathbf{G}$ is called admissible iff

- $\kappa\left(\delta_{1}\right)=\kappa\left(\delta_{2}\right)$ for all $\delta_{1}, \delta_{2} \in \mathcal{Q}$ with $\delta_{1} \uparrow \uparrow \delta_{2}$, and

- $\kappa\left(\gamma_{1}^{-1}\right)=\kappa\left(\gamma_{2}\right)$ for all $\gamma \in \mathcal{Q}$ and all decompositions $\gamma_{1} \gamma_{2}$ of $\gamma$.

Most relevant for the well-definedness of the Weyl operators to be introduced below, will be

Theorem 3.12. Let $\mathcal{Q}$ be a complete subset of $\mathcal{P}_{\text {gen }}$ and $\kappa: \mathcal{Q} \longrightarrow \mathbf{G}$ an admissible map.

Then there is a unique map $\Theta: \overline{\mathcal{A}} \longrightarrow \overline{\mathcal{A}}$, such that, for all $\gamma \in \mathcal{Q}$,

$$
h_{\Theta(\bar{A})}([\gamma])=\kappa(\gamma)^{-1} h_{\bar{A}}([\gamma]) \kappa\left(\gamma^{-1}\right) .
$$

Moreover, $\Theta$ is a homeomorphism preserving the Ashtekar-Lewandowski measure $\mu_{0}$. Hence, the pull-back $\Theta^{*}: C(\overline{\mathcal{A}}) \longrightarrow C(\overline{\mathcal{A}})$ is an isometry and the induced operator on $\mathcal{B}\left(L_{2}\left(\overline{\mathcal{A}}, \mu_{0}\right)\right)$ is well defined and unitary.

A more general version is proven in [13]. We replay the corresponding proof.

Proof. • Define $\lambda: \overline{\mathcal{A}} \longrightarrow \operatorname{Maps}(\mathcal{Q}, \mathbf{G})$ by $^{7}$

$$
(\lambda(\bar{A}))(\gamma)=\kappa(\gamma)^{-1} h_{\bar{A}}(\gamma) \kappa\left(\gamma^{-1}\right) .
$$

- First we show that $\lambda(\bar{A})$ is indeed in $\operatorname{Germ}(\mathcal{Q}, \mathbf{G})$ for all $\bar{A} \in \overline{\mathcal{A}}$.

In fact, for all $\gamma \in \mathcal{Q}$ and all decompositions $\gamma_{1} \gamma_{2}$ of $\gamma$, we have

$$
\begin{aligned}
(\lambda(\bar{A}))\left(\gamma^{-1}\right) & =\kappa\left(\gamma^{-1}\right)^{-1} h_{\bar{A}}\left(\gamma^{-1}\right) \kappa(\gamma) \\
& =\left(\kappa(\gamma)^{-1} h_{\bar{A}}(\gamma) \kappa\left(\gamma^{-1}\right)\right)^{-1}=(\lambda(\bar{A})(\gamma))^{-1}
\end{aligned}
$$

and

$$
\begin{aligned}
(\lambda(\bar{A}))(\gamma) & =\kappa(\gamma)^{-1} h_{\bar{A}}(\gamma) \kappa\left(\gamma^{-1}\right) \\
& =\kappa\left(\gamma_{1} \gamma_{2}\right)^{-1} h_{\bar{A}}\left(\gamma_{1}\right) h_{\bar{A}}\left(\gamma_{2}\right) \kappa\left(\gamma_{2}^{-1} \gamma_{1}^{-1}\right) \\
& =\kappa\left(\gamma_{1}\right)^{-1} h_{\bar{A}}\left(\gamma_{1}\right) \kappa\left(\gamma_{1}^{-1}\right) \kappa\left(\gamma_{2}\right)^{-1} h_{\bar{A}}\left(\gamma_{2}\right) \kappa\left(\gamma_{2}^{-1}\right) \\
& =(\lambda(\bar{A}))\left(\gamma_{1}\right)(\lambda(\bar{A}))\left(\gamma_{2}\right) .
\end{aligned}
$$

Here, we used the admissibility of $\kappa$ with $\gamma_{1} \uparrow \uparrow \gamma_{1} \gamma_{2}$ and $\gamma_{2}^{-1} \gamma_{1}^{-1} \uparrow \uparrow \gamma_{2}^{-1}$.

\footnotetext{
7 From now on, we will drop the square brackets in all $h_{\bar{A}}([\ldots])$.
} 
- Next, observe that, for every fixed $\gamma \in \mathcal{Q}$,

$$
(\lambda(\bar{A}))(\gamma)=\kappa(\gamma)^{-1} h_{\bar{A}}(\gamma) \kappa\left(\gamma^{-1}\right) \equiv \kappa(\gamma)^{-1} \pi_{\gamma}(\bar{A}) \kappa\left(\gamma^{-1}\right)
$$

depends continuously on $\bar{A}$, by definition of the projective-limit topology on $\overline{\mathcal{A}}$.

- Now, by Proposition 3.10, $\Theta:=\widehat{[\lambda(\cdot)]}: \overline{\mathcal{A}} \longrightarrow \overline{\mathcal{A}}$ is continuous, whereas for $\gamma \in \mathcal{Q}$,

$$
\left.h_{\Theta(\bar{A})}(\gamma) \equiv(\Theta(\bar{A}))([\gamma])=\widehat{[\lambda(\bar{A})}\right]([\gamma])=\kappa(\gamma)^{-1} h_{\bar{A}}(\gamma) \kappa\left(\gamma^{-1}\right) .
$$

The uniqueness of $\Theta$ follows from the completeness of $\mathcal{Q}$.

- To prove that $\Theta$ is a homeomorphism, we explicitly describe the inverse of $\Theta$. Define $\kappa^{\prime}: \mathcal{Q} \longrightarrow \mathbf{G}$ by $\kappa^{\prime}(\gamma):=\kappa(\gamma)^{-1}$. It is easy to check that $\kappa^{\prime}$ is admissible. As already proven above, there is a unique continuous map $\Theta^{\prime}: \overline{\mathcal{A}} \longrightarrow \overline{\mathcal{A}}$ with

$$
h_{\Theta^{\prime}(\bar{A})}(\gamma)=\kappa^{\prime}(\gamma)^{-1} h_{\bar{A}}(\gamma) \kappa^{\prime}\left(\gamma^{-1}\right)
$$

for all $\gamma \in \mathcal{Q}$. Altogether, this gives

$$
\begin{aligned}
h_{\Theta^{\prime}(\Theta(\bar{A}))}(\gamma) & =\kappa^{\prime}(\gamma)^{-1} h_{\Theta(\bar{A})}(\gamma) \kappa^{\prime}\left(\gamma^{-1}\right) \\
& =\kappa^{\prime}(\gamma)^{-1} \kappa(\gamma)^{-1} h_{\bar{A}}(\gamma) \kappa\left(\gamma^{-1}\right) \kappa^{\prime}\left(\gamma^{-1}\right) \\
& =h_{\bar{A}}(\gamma)
\end{aligned}
$$

for all $\gamma \in \mathcal{Q}$. The completeness of $\mathcal{Q}$ and Lemma 3.11 prove $\Theta^{\prime} \circ \Theta=\mathrm{id}_{\overline{\mathcal{A}}}$. Analogously, one shows $\Theta \circ \Theta^{\prime}=\mathrm{id}_{\overline{\mathcal{A}}}$.

- $\Theta$ even preserves the Ashtekar-Lewandowski measure.

In fact, let $v$ be an arbitrary, but fixed hyph. By completeness, there is some hyph $v^{\prime} \geq v$ with $Y^{\prime}$ edges and $v^{\prime} \subseteq \mathcal{Q}$. By construction, we have

$$
\pi_{v^{\prime}} \circ \Theta=\left(\Theta_{\gamma_{1}} \times \cdots \times \Theta_{\gamma_{Y^{\prime}}}\right) \circ \pi_{v^{\prime}}
$$

with $\Theta_{\gamma}(g):=\kappa(\gamma)^{-1} g \kappa\left(\gamma^{-1}\right)$ for $\gamma \in \mathcal{Q}$. In other words, each $\Theta_{\gamma}$ consists of a left and a right translation, whence the Haar measure on $\mathbf{G}$ is $\Theta_{\gamma}$-invariant. Since $\pi_{v}^{v^{\prime}} \circ \pi_{v^{\prime}}=\pi_{v}$ with continuous $\pi_{v}^{v^{\prime}}: \overline{\mathcal{A}}_{v^{\prime}} \longrightarrow \overline{\mathcal{A}}_{v}$ and since $\left(\pi_{v^{\prime}}\right)_{*} \mu_{0}$ is the $Y^{\prime}$-fold product of the Haar measure on $\mathbf{G}$, we get

$$
\begin{aligned}
\left(\pi_{v}\right)_{*}\left(\Theta_{*} \mu_{0}\right) & =\left(\pi_{v}^{v^{\prime}}\right)_{*}\left(\pi_{v^{\prime}} \circ \Theta\right)_{*} \mu_{0} \\
& =\left(\pi_{v}^{v^{\prime}}\right)_{*}\left(\Theta_{\gamma_{1}} \times \cdots \times \Theta_{\gamma_{Y^{\prime}}}\right)_{*}\left(\pi_{v^{\prime}}\right)_{*} \mu_{0} \\
& =\left(\pi_{v}^{v^{\prime}}\right)_{*}\left(\Theta_{\gamma_{1}} \times \cdots \times \Theta_{\gamma_{Y^{\prime}}}\right)_{*} \mu_{\text {Haar }}^{Y^{\prime}} \\
& =\left(\pi_{v}^{v^{\prime}}\right)_{*} \mu_{\text {Haar }}^{Y^{\prime}} \\
& =\left(\pi_{v}^{v^{\prime}}\right)_{*}\left(\pi_{v^{\prime}}\right)_{*} \mu_{0} \\
& =\left(\pi_{v}\right)_{*} \mu_{0} .
\end{aligned}
$$

Since finite regular Borel measures on $\overline{\mathcal{A}}$ coincide iff their push-forwards w.r.t. all $\pi_{v}$ coincide, we get the assertion.

We get immediately

Corollary 3.13. Let $\mathcal{Q}$ be some complete subset of $\mathcal{P}_{\text {gen }}$. Moreover, let $Y$ be some topological space and let $\kappa: \mathcal{Q} \times Y \longrightarrow \mathbf{G}$ be some map, such that 
- $\kappa(\cdot, y): \mathcal{Q} \longrightarrow \mathbf{G}$ is admissible for all $y \in Y$, and

- $\kappa(\gamma, \cdot): Y \longrightarrow \mathbf{G}$ is continuous for all $\gamma \in \mathcal{Q}$.

Then there is a unique map $\Theta: \overline{\mathcal{A}} \times Y \longrightarrow \overline{\mathcal{A}}$ with

$$
h_{\Theta(\bar{A}, y)}(\gamma)=\kappa(\gamma, y)^{-1} h_{\bar{A}}(\gamma) \kappa\left(\gamma^{-1}, y\right)
$$

for all $\gamma \in \mathcal{Q}$. Moreover, $\Theta$ is continuous.

3.3. Surfaces and fluxes. Originally (see, e.g., [32]), the action of flux operators on cylindrical functions has been given by self-adjoint differential operators. Since these operators are unbounded, one has to study their domains very carefully. To avoid this problem, one usually considers them as generators of unitary, i.e., bounded operators. Now, the flux operators turn into some sort of translation operators. In this section, we are going to shift this action to a still deeper level. We will see that it can be regarded as the pull-back of some continuous action of translations on $\overline{\mathcal{A}}$ itself.

3.3.1. Quasi-surfaces. Before we can define this action we study how paths are decomposed by surfaces.

Definition 3.8. Let $S$ be a subset of $M$.

- A path $\gamma \in \mathcal{P}_{\text {gen }}$ is called $S$-external iff (int $\left.\gamma\right) \cap S=\varnothing$.

- A path $\gamma \in \mathcal{P}_{\text {gen }}$ is called $S$-internal iff int $\gamma \subseteq S$.

Observe that the end points of an $S$-external path may be contained in $S$. It is only required for the "interior part" of the path, i.e., for all $\gamma(t)$ with $0<t<1$ to be outside of $S$. If $S$ is clear from the context, we simply speak about external and internal edges.

Definition 3.9. Let $S$ be some subset of $M$.

Then $\mathcal{Q}_{S}$ denotes the set of all paths that are $S$-external or $S$-internal.

Definition 3.10. Let $S$ be a subset of $M$ and $\gamma \in \mathcal{P}_{\text {gen }}$ be an edge.

Then a decomposition $\gamma$ of $\gamma$ is called $S$-admissible iff $\gamma \subseteq \mathcal{Q}_{S}$.

In other words, $\boldsymbol{\gamma}=\left(\gamma_{1}, \ldots, \gamma_{I}\right)$ is $S$-admissible iff $\gamma$ equals $\gamma_{1} \cdots \gamma_{I}$ up to the parametrization and each $\gamma_{i}$ is $S$-internal or $S$-external.

Lemma 3.14. Let $S$ be a subset of $M$.

Then $\mathcal{Q}_{S}$ is complete, if every edge has an $S$-admissible decomposition.

Proof. Heredity is clear. The completeness follows since any (finite) path can be decomposed into a product of edges and trivial paths, hence, by assumption, into a product of $S$-external or $S$-internal paths.

Definition 3.11. A subset $S$ of $M$ is called quasi-surface iff every edge $\gamma \in \mathcal{P}_{\text {gen }}$ has an $S$-admissible decomposition. 
Examples for quasi-surfaces, in case we are in the (semi)analytic category for the paths, are embedded analytic submanifolds that are even semianalytic. ${ }^{8}$ Note that these submanifolds may have any dimension. Therefore, any collection of points having no accumulation point is a quasi-surface. This even remains true in the category of piecewise smooth paths.

On the other hand, there are indeed non-semianalytic submanifolds that are quasisurfaces. Consider, e.g., the smooth function $f$ on $\mathbb{R}$ with $f(x):=\mathrm{e}^{-1 / x^{2}}$ for $x \neq 0$ and $f(0):=0$. Of course, it is analytic everywhere except for $x=0$. But, its graph $S$ does not form a semianalytic submanifold in, for simplicity, $\mathbb{R}^{2}$. Nevertheless, it is a quasi-surface. In fact, let $\gamma$ be a piecewise analytic path in $\mathbb{R}^{2}$. If it does not run through the origin, the statement is trivial. Assume now that $\gamma$ runs through the origin. Decomposing $\gamma$ appropriately, if necessary, we may restrict ourselves to the case of an analytic $\gamma$ starting at the origin without returning there at any other parameter time. Assume next that $\gamma$ has infinitely many intersection points with $S$, and let the origin 0 be an accumulation point for int $\gamma \cap S$. W.1.o.g., ${ }^{9}$ we may consider, finally, $\gamma$ to be the graph of an analytic function on $\mathbb{R}$, again denoted by $\gamma$. Use now the fact that two $C^{\infty}$ functions $f_{1}, f_{2}$ have identical Taylor coefficients at 0 if 0 is an accumulation point of $f_{1}=f_{2}$, to derive that $\gamma$ has only zero Taylor coefficients, just because $f$ does. Now, analyticity implies that $\gamma$ is a straight edge along the $x$-axis never intersecting $S$ again. Using this contradiction, the statement is now trivial.

If we would like to take even more quasi-surfaces into account, we may reduce the set of paths under consideration. This might be relevant, e.g., in the case of piecewise linear paths, although there usually also the set of manifolds is restricted to that of piecewise linear submanifolds a priori.

The punctures leading to an $S$-admissible decomposition will be relevant for the definition of Weyl operators. In particular, these operators depend on the transversality properties between the path and the (oriented) hypersurface. Therefore, we need to introduce a general notion for the properties an orientation should encode.

Definition 3.12. Let $S$ be a quasi-surface of $M$.

- A function $\sigma_{S}: \mathcal{P}_{\text {gen }} \longrightarrow \mathbb{Z}$ is called

- outgoing intersection function for $S$ iff we have

1. $\sigma_{S}(\gamma)=0$ if $\gamma(0) \notin S$ and

2. $\sigma_{S}(\gamma)=\sigma_{S}\left(\gamma^{\prime}\right)$

for all $\gamma, \gamma^{\prime} \in \mathcal{P}_{\text {gen }}$ with $\gamma \uparrow \uparrow \gamma^{\prime}$;

- incoming intersection function for $S$ iff we have

1. $\sigma_{S}(\gamma)=0$ if $\gamma(1) \notin S$ and

2. $\sigma_{S}(\gamma)=\sigma_{S}\left(\gamma^{\prime}\right)$

for all $\gamma, \gamma^{\prime} \in \mathcal{P}_{\text {gen }}$ with $\gamma \downarrow \downarrow \gamma^{\prime}$.

- An outgoing intersection function $\sigma_{S}^{-}$and an incoming intersection function $\sigma_{S}^{+}$are called compatible iff $\sigma_{S}^{-}(\gamma)+\sigma_{S}^{+}\left(\gamma^{-1}\right)=0$ for all $\gamma \in \mathcal{P}_{\text {gen }}$.

\footnotetext{
8 This, however, is no longer true if we drop the semianalyticity (for its definition see Subsect. 6.3). In fact, consider $\mathbb{R}^{2}$ and a smooth path $\gamma$ in the closed half-plane $y \leq 0$, such that $\gamma$ connects $(-1,0)$ and $(+1,0)$ and intersects the straight line $\delta$ between these two points infinitely often without sharing a full segment. (See similar constructions, e.g., in [7,17].). Now define $S$ to be the upper one of the two open sets in $\mathbb{R}^{2}$ bounded by $\gamma$, by $x=-1$ and by $x=+1$. Of course, $S$ is an embedded analytic manifold, although it is not semianalytic in $\mathbb{R}^{2}$. Nevertheless, $\delta$ leaves $S$ and returns into it infinitely often. Therefore, there is no $S$-admissible decomposition of $\delta$, whence $S$ is not a quasi-surface.

9 Otherwise, restrict the domain of $\gamma$, such that the $x$-component of $\dot{\gamma}$ is non-zero everywhere. If this is not possible, the $x$-component of $\dot{\gamma}$ vanishes at $t=0$. But, then $\gamma$ is $S$-external anyway, at least locally.
} 
For brevity, we will denote a compatible pair $\left(\sigma_{S}^{-}, \sigma_{S}^{+}\right)$of an outgoing and an incoming intersection function by $\sigma_{S}$ and call it intersection function for $S$. Even more, we use $\sigma_{S}$ and $\sigma_{S}^{-}$synonymously. Sometimes, we write $\sigma(S, \gamma)$ instead of $\sigma_{S}(\gamma)$ to emphasize that the intersection function may depend on quasi-surface and path as well.

Definition 3.13. Let $S$ be a quasi-surface of $M$, and let $\sigma_{S}: \mathcal{P}_{\text {gen }} \longrightarrow \mathbb{Z}$ be some intersection function for $S$.

Then the intersection function $-\sigma_{S}$ is called inverse to $\sigma_{S}$.

Definition 3.14. Let $S$ be a quasi-surface with intersection function $\sigma_{S}$, and let $\gamma \in \mathcal{P}_{\text {gen }}$ be some path. Assume, moreover, that there are only finitely many $\tau_{i} \in[0,1]$ with $\gamma\left(\tau_{i}\right) \in S$.

We say that the orientation of $S$ coincides with the direction of $\gamma$ iff $\sigma_{S}^{-}\left(\left.\gamma\right|_{\left[\tau_{i}, 1\right]}\right)=1$ for all $\tau_{i} \neq 1$ and $\sigma_{S}^{+}\left(\left.\gamma\right|_{\left[0, \tau_{i}\right]}\right)=1$ for all $\tau_{i} \neq 0$.

In our applications, we will, e.g., define $\sigma_{S}(\gamma)$ for an $S$-external path $\gamma$ to be \pm 1 (depending on the direction of $\gamma$ ), if its initial path intersects $S$ transversally, and equal to 0 , otherwise:

Definition 3.15. Let $S$ be an oriented (embedded) hypersurface in $M$ being a quasisurface of $M$. Then we have:

1. The natural intersection function $\sigma_{S}: \mathcal{P}_{\text {gen }} \longrightarrow \mathbb{Z}$ is defined by:

- $\sigma_{S}(\gamma)=0$ if $\gamma(0) \notin S$ or $\dot{\gamma}(0)$ is tangent to $S$;

- $\sigma_{S}(\gamma)= \pm 1$ if $\gamma(0) \in S$ and $\dot{\gamma}(0)$ is not tangent to $S$ and some initial path of $\gamma$ lies (except $\gamma(0)$ ) above (below) $S$.

2. The topological intersection function $\sigma_{S}^{\text {top }}: \mathcal{P}_{\text {gen }} \longrightarrow \mathbb{Z}$ is defined as follows:

- $\sigma_{S}^{\text {top }}(\gamma)=0$ if $\gamma(0) \notin S$ or some initial path of $\gamma$ is contained in $S$;

- $\sigma_{S}^{\text {top }}(\gamma)= \pm 1$ if $\gamma(0) \in S$ and no initial path of $\gamma$ is contained in $S$ and some initial path of $\gamma$ lies (except $\gamma(0))$ above (below) $S$.

Here, "above" and "below" refer to the orientation of $S$. Moreover, initial paths w.r.t. a trivial interval are not taken into consideration. It is easy to check that this definition is well defined. Moreover, obviously, for every orientable $S$ there are precisely two natural (and two topological) intersection functions corresponding to the two choices of orientations. They coincide up to the sign.

If $S$ is a submanifold of codimension larger than 1, there is no longer just a pair of natural orientations. Nevertheless, in view of the applications we aim at, we may define "natural" orientations:

Definition 3.16. Let $S$ be some embedded submanifold of $M$ being a quasi-surface of $M$ and having codimension 2 or higher.

Then an intersection function $\sigma_{S}: \mathcal{P}_{\text {gen }} \longrightarrow \mathbb{Z}$ is called natural (topological) iff there is some oriented embedded hypersurface $S^{\prime}$ in $M$ being a quasi-surface and having $\sigma_{S}$ as its natural (topological) intersection function.

One sees immediately that the number of natural intersection functions of such quasisurfaces with higher codimension may be rather large. For instance, let $S$ be (a bounded part of) a line in $\mathbb{R}^{3}$. Then we may take all the full circles in $\mathbb{R}^{3}$ having $S$ as its diameter. Of course, there is a continuum of such circles each having another pair of natural or topological intersection functions. 
Definition 3.17. • A quasi-surface $S^{\prime}$ is called quasi-subsurface of some quasi-surface $S$ iff $S^{\prime}$ is contained in $S$.

- Let $S^{\prime}$ be a quasi-subsurface of a quasi-surface $S$ having intersection function $\sigma_{S}$. Then an intersection function $\sigma_{S^{\prime}}$ is called induced by $\sigma_{S}$ iff $\sigma_{S}(\gamma)=\sigma_{S^{\prime}}(\gamma)$ for all $\gamma$ with $\gamma(0) \in S^{\prime}$.

Definition 3.16 gives an example for the induction of intersection functions.

Lemma 3.15. The complement of a quasi-surface is a quasi-surface.

Proof. An $S$-admissible decomposition of an edge is also $(M \backslash S)$-admissible.

Lemma 3.16. If $S_{1}$ and $S_{2}$ are quasi-surfaces, then $S_{1} \cup S_{2}$ and $S_{1} \cap S_{2}$ are quasi-surfaces.

Proof. If $\gamma$ is some edge, decompose each path of some $S_{1}$-admissible decomposition w.r.t. $S_{2}$. It is easy to check that this leads to an $S$-admissible decomposition of $\gamma$ with $S$ being $S_{1} \cup S_{2}$ or $S_{1} \cap S_{2}$.

Corollary 3.17. Let $S_{1}$ and $S_{2}$ be quasi-surfaces with intersection functions $\sigma_{S_{1}}$ and $\sigma_{S_{2}}$, respectively. Then $\sigma_{S_{1}}+\sigma_{S_{2}}$ is an intersection function for $S:=S_{1} \cup S_{2}$. If, additionally, $\sigma_{S_{1}}$ and $\sigma_{S_{2}}$ coincide for all paths starting at $S_{1} \cap S_{2}$, then the function $\sigma_{S_{1} S_{2}}$ defined by

$$
\sigma_{S_{1} S_{2}}(\gamma):= \begin{cases}\sigma_{S_{1}}(\gamma) & \text { if } \gamma(0) \in S_{1}, \\ 0 & \text { if } \gamma(0) \notin S_{1} \cup S_{2}, \\ \sigma_{S_{2}}(\gamma) & \text { if } \gamma(0) \in S_{2},\end{cases}
$$

is an intersection function for $S$. It is called joint intersection function.

Obviously, the joint intersection function equals $\sigma_{S_{1}}+\sigma_{S_{2}}$ if $S_{1}$ and $S_{2}$ are disjoint.

Sometimes, it is convenient to use some sort of standard decomposition of edges. Indeed, there is a minimal decomposition.

Definition 3.18. Let $S$ be a subset of $M$ and $\gamma \in \mathcal{P}_{\text {gen }}$ be an edge.

An $S$-admissible decomposition $\boldsymbol{\gamma}$ of $\gamma$ is called minimal iff $\boldsymbol{\gamma}^{\prime} \geq \boldsymbol{\gamma}$ for any other $S$-admissible decomposition $\boldsymbol{\gamma}^{\prime}$ of $\gamma$.

In other words, $\boldsymbol{\gamma}=\left(\gamma_{1}, \ldots, \gamma_{I}\right)$ is minimal iff every other $S$-admissible decomposition $\boldsymbol{\gamma}^{\prime}=\left(\gamma_{1}^{\prime}, \ldots, \gamma_{J}^{\prime}\right)$ is a refinement of $\boldsymbol{\gamma}$, i.e., there are $0=j_{0}<j_{1}<\cdots<j_{I}=J$, such that $\gamma_{i}$ equals $\gamma_{j_{i-1}+1}^{\prime} \cdots \gamma_{j_{i}}^{\prime}$ up to the parametrization.

Lemma 3.18. If an edge $\gamma$ has any $S$-admissible decomposition, it has also a minimal $S$-admissible decomposition. Moreover, this minimal decomposition is unique up to the parametrization of its components.

Proof. Let $\delta$ be an $S$-admissible decomposition of $\gamma$. Since $\gamma$ equals $\delta_{1} \cdots \delta_{K}$ up to the parametrization, the parameter domain $[0,1]$ of $\gamma$ may be decomposed into nontrivial closed intervals $R_{k}=\left[t_{k-1}, t_{k}\right] \subseteq[0,1]$, such that each $\left.\gamma\right|_{R_{k}}$ corresponds to $\delta_{k}$. Cancel now in $T^{\prime}:=\left\{t_{0}, t_{1}, \ldots, t_{K}\right\}$ each $t_{k} \neq 0,1$ with int $\left.\gamma\right|_{\left[t_{k-1}, t_{k+1}\right]} \cap S=\varnothing$ or int $\left.\gamma\right|_{\left[t_{k-1}, t_{k+1}\right]} \subseteq S$. The remaining set $T=\left\{\tau_{0}, \ldots, \tau_{I}\right\} \subseteq T^{\prime}$ naturally defines another $S$-admissible decomposition $\gamma=\left(\gamma_{1}, \ldots, \gamma_{I}\right)$ of $\gamma$ and a corresponding decomposition of $[0,1]$ into intervals $P_{i}$.

Let now $\boldsymbol{\gamma}^{\prime}=\left(\gamma_{1}^{\prime}, \ldots, \gamma_{J}^{\prime}\right)$ be any $S$-admissible decomposition of $\gamma$. Then each $\gamma_{i}^{\prime}$ corresponds to some interval $Q_{j} \subseteq[0,1]$ with $\left.\gamma\right|_{Q_{j}}=\gamma_{j}^{\prime}$. Assume that $Q_{j}$ overlaps two different intervals $P_{i}$ and $P_{i+1}$, i.e., $\gamma\left(\tau_{i}\right) \in$ int $\gamma_{j}^{\prime}$. 
- Let $\gamma\left(\tau_{i}\right) \in S$. Then int $\gamma_{j}^{\prime}=$ int $\left.\gamma\right|_{Q_{j}} \subseteq S$, hence int $\left.\gamma\right|_{P_{i}} \subseteq S$ and int $\left.\gamma\right|_{P_{i+1}} \subseteq S$, by admissibility. Consequently, int $\left.\gamma\right|_{P_{i} \cup P_{i+1}}=$ int $\left.\gamma\right|_{P_{i}} \cup\left\{\gamma\left(\tau_{i}\right)\right\} \cup$ int $\left.\gamma\right|_{P_{i+1}} \subseteq S$. This implies $\tau_{i} \notin T$, in contradiction to the minimality of $\gamma$.

- Let $\gamma\left(\tau_{i}\right) \notin S$. Then, analogously, we get a contradiction.

Consequently, $Q_{j}$ can overlap nontrivially only either $P_{i}$ or $P_{i+1}$.

Definition 3.19. Let $S$ be a quasi-surface with intersection function $\sigma_{S}$, let $\gamma$ be an edge and let $\boldsymbol{\gamma}=\left\{\gamma_{i}\right\}_{i=0}^{n}$ be its minimal $S$-admissible decomposition.

Then a point $x \in M$ is called

- $\gamma$-puncture in $S$ iff there is an $i \in[1, n]$ with

$$
\gamma_{i-1}(1)=x=\gamma_{i}(0) \quad \text { and } \quad \sigma_{S}^{+}\left(\gamma_{i-1}\right) \sigma_{S}^{-}\left(\gamma_{i}\right)>0
$$

- $\gamma$-half-puncture in $S$ iff there is an $i \in[0, n]$ with

$$
x=\gamma_{i}(0) \quad \text { and } \quad \sigma_{S}^{-}\left(\gamma_{i}\right) \neq 0
$$

or

$$
x=\gamma_{i}(1) \quad \text { and } \quad \sigma_{S}^{+}\left(\gamma_{i}\right) \neq 0 .
$$

We say that $\gamma$ intersects $S$ completely transversally iff there are no $S$-internal edges in the minimal $S$-admissible decomposition of $\gamma$ and each $\gamma$-half-puncture is also a $\gamma$-puncture.

Roughly speaking, $x$ is a $\gamma$-puncture iff $\gamma$ intersects $S$ (w.r.t. $\sigma_{S}$ ) transversally at $x$.

3.3.2. Quasi-flux action. In this subsection, $S$ is some quasi-surface and $\sigma_{S}$ some intersection function for $S$.

Proposition 3.19. There is a unique map $\Theta^{S, \sigma_{S}}: \overline{\mathcal{A}} \times \operatorname{Maps}(M, \mathbf{G}) \longrightarrow \overline{\mathcal{A}}$, such that

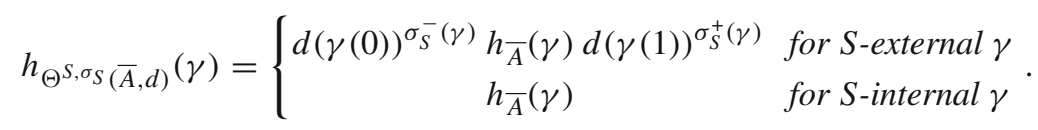

If $\operatorname{Maps}(M, \mathbf{G}) \cong \mathbf{G}^{M}$ is given the product topology, then $\Theta$ is continuous. Moreover, the map

$$
\Theta_{d}^{S, \sigma_{S}}: \overline{\mathcal{A}} \longrightarrow \overline{\mathcal{A}}
$$

given by $\Theta_{d}^{S, \sigma_{S}}(\bar{A}):=\Theta^{S, \sigma_{S}}(\bar{A}, d)$, is a homeomorphism and preserves the AshtekarLewandowski measure for each $d \in \operatorname{Maps}(M, \mathbf{G})$. Finally, the inverse of $\Theta_{d}^{S, \sigma_{S}}$ is given by $\Theta_{d^{-1}}^{S, \sigma_{S}}$. 
Proof. - $\Theta^{S, \sigma_{S}}$ exists uniquely and is continuous for the product topology on $\operatorname{Maps}(M, \mathbf{G})$.

First note that $\mathcal{Q}_{S}$ is complete by Lemma 3.14. Let now $Y:=\operatorname{Maps}(M, \mathbf{G})$ and define

$$
\kappa(\gamma, d):=\left\{\begin{array}{ll}
d(\gamma(0))^{-\sigma_{S}^{-}(\gamma)} & \text { if } \gamma \text { is } S \text {-external } \\
e_{\mathbf{G}} & \text { if } \gamma \text { is } S \text {-internal }
\end{array} .\right.
$$

The only nontrivial property of $\kappa$ in Corollary 3.13 to be checked is $\kappa\left(\gamma_{1}^{-1}, d\right)=$ $\kappa\left(\gamma_{2}, d\right)$ for decompositions $\gamma_{1} \gamma_{2}$ of $S$-external $\gamma$. Observe, however, that here $\gamma_{1}^{-1}(0) \equiv \gamma_{1}(1) \equiv \gamma_{2}(0)$ is not contained in $S$, hence $\kappa\left(\gamma_{1}^{-1}, d\right)=e_{\mathbf{G}}=\kappa\left(\gamma_{2}, d\right)$. The claim now follows from $\sigma_{S}^{-}(\gamma)+\sigma_{S}^{+}\left(\gamma^{-1}\right)=0$ and Corollary 3.13.

- $\Theta_{d}^{S, \sigma_{S}}$ is a homeomorphism and leaves $\mu_{0}$ invariant.

This now follows from Theorem 3.12.

Definition 3.20. $\Theta^{S, \sigma_{S}}: \overline{\mathcal{A}} \times \operatorname{Maps}(M, \mathbf{G}) \longrightarrow \overline{\mathcal{A}}$ is called quasi-flux action.

Remark. Note that $\Theta^{S, \sigma_{S}}$ is, in general, not a group action of $\operatorname{Maps}(M, \mathbf{G})$.

But, we have

Lemma 3.20. Let $S_{1}$ and $S_{2}$ be two quasi-surfaces, and let $d_{1}, d_{2}: M \longrightarrow \mathbf{G}$ be functions commuting on $S_{1} \cap S_{2}$. Let $d: M \longrightarrow \mathbf{G}$ be any function with

$$
d:= \begin{cases}d_{1} & \text { on } S_{1} \backslash S_{2} \\ d_{1} d_{2} & \text { on } S_{1} \cap S_{2} \\ d_{2} & \text { on } S_{2} \backslash S_{1}\end{cases}
$$

If $\sigma_{S_{1}}$ and $\sigma_{S_{2}}$ coincide for all paths starting in $S_{1} \cap S_{2}$ and vanish both for $S_{1}$ - and $S_{2}$-internal paths, then

$$
\Theta_{d_{1}}^{S_{1}, \sigma_{S_{1}}} \circ \Theta_{d_{2}}^{S_{2}, \sigma_{S_{2}}}=\Theta_{d}^{S_{1} \cup S_{2}, \sigma_{S_{1} S_{2}}}=\Theta_{d_{2}}^{S_{2}, \sigma_{S_{2}}} \circ \Theta_{d_{1}}^{S_{1}, \sigma_{S_{1}}} .
$$

Proof. By direct calculation.

Corollary 3.21. Let $d_{1}, d_{2}: M \longrightarrow \mathbf{G}$ be two functions.

If $d_{1}$ and $d_{2}$ commute pointwise, we have $\Theta_{d_{1}}^{S, \sigma_{S}} \circ \Theta_{d_{2}}^{S, \sigma_{S}}=\Theta_{d_{1} d_{2}}^{S, \sigma_{S}}$.

Proof. Straightforward.

3.4. Weyl operators. Recall that every continuous map $\psi: X \longrightarrow X$ on a topological space $X$ defines a continuous pull-back map $\psi^{*}: C(X) \longrightarrow C(X)$. This map is an isometry if $\psi$ is surjective. If $X$ is even a compact Hausdorff space, $\psi$ is surjective and $\mu$ a (finite) regular Borel measure on $X$ with $\psi_{*} \mu=\mu$, then $\psi^{*}$ is a unitary operator on $L_{2}(X, \mu)$. This motivates

Definition 3.21. The operators

$$
w_{d}^{S, \sigma_{S}}:=\left(\Theta_{d}^{S, \sigma_{S}}\right)^{*}
$$

with $S$ being a quasi-surface, $\sigma_{S}$ an intersection function and $d: M \longrightarrow \mathbf{G}$ being any function are called Weyl operators.

Note that each Weyl operator is both a map on $C(\overline{\mathcal{A}})$ and $L_{2}\left(\overline{\mathcal{A}}, \mu_{0}\right)$. In fact, Proposition 3.19 gives 
Proposition 3.22. • Every Weyl operator is an isometry on $C(\overline{\mathcal{A}})$.

- Every Weyl operator is a unitary operator on $L_{2}\left(\overline{\mathcal{A}}, \mu_{0}\right)$.

Note, however, that measures, in general, lead to Weyl operators that are ill defined on the $L_{2}$-functions: For instance, let us work in the analytic category, fix some hypersurface $S$ and some intersection function $\sigma_{S}$. Assume now that, $g$ running over $\mathbf{G}$, we have all Weyl operators at our disposal that are given by

$$
w_{g}^{S, \sigma_{S}}:=w_{d_{g}}^{S, \sigma_{S}},
$$

where $d_{g}$ is the constant function on $M$ with value $g \in \mathbf{G}$. To make all these Weyl operators well defined as operators on $L_{2}(\overline{\mathcal{A}}, \mu)$ for some $\mu$, we have at least to demand that, for each $S$-external edge $\gamma$ (having only one end attached to $S$ ), the support of the push-forward measure $\left(\pi_{\gamma}\right)_{*} \mu$ equals $\mathbf{G}$. Of course, there are many measures without this property.

Let us now collect some additional properties of Weyl operators, again following directly from the properties of $\Theta$ and the definition of Weyl operators by pull-backs.

Lemma 3.23. Let $S$ be a quasi-surface and let $d, d_{1}, d_{2}: M \longrightarrow \mathbf{G}$ be some functions. Then we have (dropping always the upper indices $S, \sigma_{S}$ in $w_{d}^{S, \sigma_{S}}$ ):

1. $w_{d}\left(f_{1} f_{2}\right)=w_{d}\left(f_{1}\right) w_{d}\left(f_{2}\right)$ for all functions $f_{1}, f_{2}$ on $\overline{\mathcal{A}}$.

2. $w_{d_{1}} w_{d_{2}}=w_{d_{1} d_{2}}$, if $d_{1} d_{2}=d_{2} d_{1}$.

Corollary 3.24. For all quasi-surfaces $S$, all intersection functions $\sigma_{S}$ and all functions $d: M \longrightarrow \mathbf{G}$, we have

$$
w_{d}^{S,-\sigma_{S}}=w_{d^{-1}}^{S, \sigma_{S}}=\left(w_{d}^{S, \sigma_{S}}\right)^{-1} \equiv\left(w_{d}^{S, \sigma_{S}}\right)^{*} .
$$

The preceding corollary implies that the inversion of the orientation of a quasi-surface leads to the adjoint Weyl operator. The uniqueness proof in Sect. 7 will heavily use this fact.

Corollary 3.25. Let $v=\left\{\gamma_{1}, \ldots, \gamma_{n}\right\}$ be a hyph. Then

$$
w_{d}^{S, \sigma_{S}}\left(T_{1} \otimes \cdots \otimes T_{n}\right)=w_{d}^{S, \sigma_{S}}\left(T_{1}\right) \otimes \cdots \otimes w_{d}^{S, \sigma_{S}}\left(T_{n}\right)
$$

for all $T_{i} \in \mathcal{M}_{\gamma_{i}}$ and all functions $d: M \longrightarrow \mathbf{G}$.

Corollary 3.17 implies

Lemma 3.26. Let $S_{1}$ and $S_{2}$ be disjoint quasi-surfaces with intersection functions $\sigma_{S_{1}}$ and $\sigma_{S_{2}}$, respectively. Let, moreover, $d_{1}, d_{2}: M \longrightarrow \mathbf{G}$ be some functions.

Then we have

$$
w_{d_{1}}^{S_{1}, \sigma_{S_{1}}} \circ w_{d_{2}}^{S_{2}, \sigma_{S_{2}}}=w_{d_{2}}^{S_{2}, \sigma_{S_{2}}} \circ w_{d_{1}}^{S_{1}, \sigma_{S_{1}}} .
$$

Lemma 3.27. Let $v$ be a hyph and $w$ be a Weyl operator for some quasi-surface $S$.

Then there is a hyph $v^{\prime} \geq v$ with $w\left(\mathcal{M}_{v}\right) \subseteq$ span $\mathcal{M}_{v^{\prime}}$. If, moreover, $v$ contains $S$-external and $S$-internal edges only, then $w\left(\mathcal{M}_{v}\right) \subseteq \operatorname{span} \mathcal{M}_{v}$.

Proof. Choose a hyph $v^{\prime} \geq v$ containing $S$-external and $S$-internal edges only. One checks immediately, that $w\left(\mathcal{M}_{v^{\prime}}\right) \subseteq \operatorname{span} \mathcal{M}_{v^{\prime}}$. Using $\mathcal{M}_{v} \subseteq \operatorname{span} \mathcal{M}_{v^{\prime}}$ (Lemma 3.2), we get the assertion. 
3.5. Regularity.

Proposition 3.28. Fix some quasi-surface $S$ and some intersection function for $\sigma_{S}$. Next, let $\Lambda_{0}$ be a set of sequential $\operatorname{Maps}(M, \mathbf{G})$-functions, such that ${ }^{10}$

$$
\mathrm{pr}_{x} \circ \lambda_{0}: \operatorname{dom} \lambda_{0} \longrightarrow \mathbf{G}
$$

is sequentially continuous for every $x \in M$ and each $\lambda_{0} \in \Lambda_{0}$. Finally, assign to each $\lambda_{0}$ some

$$
\begin{aligned}
\lambda: \operatorname{dom} \lambda_{0} & \longrightarrow \mathcal{W}, \\
y & \longmapsto w_{\lambda_{0}(y)}^{S, \sigma_{S}}
\end{aligned}
$$

$\mathcal{W}$ being the set of Weyl operators, and collect all such $\lambda$ into $\Lambda$.

Then

$$
\lambda(\cdot) \psi: \operatorname{dom} \lambda_{0} \longrightarrow \mathfrak{H}_{\mathrm{aux}} \equiv L_{2}\left(\overline{\mathcal{A}}, \mu_{0}\right)
$$

is continuous for all $\psi \in \mathfrak{H}_{\text {aux }}$ and each $\lambda \in \Lambda$.

Proof. Fix some $\lambda \in \Lambda$ with corresponding $\lambda_{0} \in \Lambda_{0}$ and recall that sequential continuity equals continuity, if the domain is sequential. To avoid cumbersome notation, we write shortly $w_{y}$ instead of $w_{\lambda_{0}(y)}^{S, \sigma_{S}}$.

- Of course, $w_{y}(\mathbf{1})=\mathbf{1}$ for all $y$.

- Let $\gamma$ be an edge and $T \in \mathcal{M}_{\gamma}$ some gauge-variant spin network state over $\gamma$.

- If $\gamma$ is internal, then $w_{y}(T)=T$ for all $y$, hence $y \longmapsto w_{y}(T)$ is continuous.

- If $\gamma$ is external, then with $T=\sqrt{\operatorname{dim} \phi} \phi_{l}^{k}$ and after a straightforward calculation, we have

$$
\begin{aligned}
&\left\|w_{y}(T)-w_{y^{\prime}}(T)\right\|_{\mathfrak{H}_{\text {aux }}}^{2} \\
&=2-2 \operatorname{Re} \phi_{k}^{k}\left(\left[\lambda_{0}\left(y^{\prime}\right)\right](\gamma(0))^{\sigma_{S}^{-}(\gamma)}\left[\lambda_{0}(y)\right](\gamma(0))^{-\sigma_{S}^{-}(\gamma)}\right) . \\
& \cdot \phi_{l}^{l}\left(\left[\lambda_{0}(y)\right](\gamma(1))^{-\sigma_{S}^{+}(\gamma)}\left[\lambda_{0}\left(y^{\prime}\right)\right](\gamma(1))^{\sigma_{S}^{+}(\gamma)}\right) \\
& \equiv 2-2 \operatorname{Re} \phi_{k}^{k}\left(\left[\operatorname{pr}_{\gamma(0)} \circ \lambda_{0}\right]\left(y^{\prime}\right)^{\sigma_{S}^{-}(\gamma)}\left[\operatorname{pr}_{\gamma(0)} \circ \lambda_{0}\right](y)^{-\sigma_{S}^{-}(\gamma)}\right) . \\
& \cdot \phi_{l}^{l}\left(\left[\operatorname{pr}_{\gamma(1)} \circ \lambda_{0}\right](y)^{-\sigma_{S}^{+}(\gamma)}\left[\operatorname{pr}_{\gamma(1)} \circ \lambda_{0}\right]\left(y^{\prime}\right)^{\sigma_{S}^{+}(\gamma)}\right) .
\end{aligned}
$$

(There is no summation over $k$ and $l$.) Since, by assumption each $\operatorname{pr}_{x} \circ \lambda_{0}$ is a continuous mapping from dom $\lambda_{0}$ to $\mathbf{G}$, we get $\left\|w_{y}(T)-w_{y^{\prime}}(T)\right\|_{\mathfrak{H}_{\text {aux }}} \rightarrow 0$ for $y \rightarrow y^{\prime}$, implying the desired continuity of $y \longmapsto w_{y}(T)$.

- Let $v$ contain external and internal edges only. Let, moreover, $T=T_{1} \otimes \cdots \otimes T_{Y}$ be in $\mathcal{M}_{v}$. Then we have

$$
\begin{aligned}
& \left\|w_{y}(T)-w_{y^{\prime}}(T)\right\|_{\mathfrak{H}_{\text {aux }}}^{2} \\
& \quad=2-2 \operatorname{Re}\left\langle w_{y} T, w_{y^{\prime}} T\right\rangle_{\mathfrak{H}_{\text {aux }}} \\
& =2-2 \operatorname{Re}\left\langle w_{y} T_{1} \otimes \cdots \otimes w_{y} T_{Y}, w_{y^{\prime}} T_{1} \otimes \cdots \otimes w_{y^{\prime}} T_{Y}\right\rangle_{\mathfrak{H}_{\text {aux }}} \\
& =2-2 \operatorname{Re} \prod_{i}\left\langle w_{y} T_{i}, w_{y^{\prime}} T_{i}\right\rangle_{\mathfrak{H}_{\text {aux }}} \\
& \quad \rightarrow 2-2 \operatorname{Re} \prod_{i}\left\langle w_{y^{\prime}} T_{i}, w_{y^{\prime}} T_{i}\right\rangle_{\mathfrak{H}_{\text {aux }}}\left(w_{y} T_{i} \rightarrow w_{y^{\prime}} T_{i} \text { by the preceding step }\right) \\
& =0
\end{aligned}
$$

${ }^{10}$ Here, $\operatorname{pr}_{x}: \operatorname{Maps}(M, \mathbf{G}) \longrightarrow \mathbf{G}$ assigns to each function from $M$ to $\mathbf{G}$ its value in $x$. 
for $y \rightarrow y^{\prime}$. The factorization of the scalar products was possible, because $w_{y}$ leaves the span of (non-trivial) matrix functions over $\gamma_{i}$ invariant and because such spans are orthogonal w.r.t. $\mu_{0}$ for paths in a hyph.

- Let now $T \in \mathcal{M}_{\mathrm{SN}}$ be an arbitrary gauge-variant spin network function, i.e., there is a hyph $v$ with $T \in \mathcal{M}_{v}$. Then there is some hyph $v^{\prime} \geq v$ containing external and internal edges only. Since $\mathcal{M}_{v} \subseteq \operatorname{span} \mathcal{M}_{v^{\prime}}$ by Lemma 3.2, $w_{y}(T) \rightarrow w_{y^{\prime}} T$ for $y \rightarrow y^{\prime}$.

- Now, Lemma A.1 gives the proof: The span of $\mathcal{M}_{\mathrm{SN}}=\bigcup_{v} \mathcal{M}_{v}$ is dense in $L_{2}\left(\overline{\mathcal{A}}, \mu_{0}\right)$, and $\left\|w_{y}\right\|=1$ for all $y$ by unitarity.

A typical example is given by the continuous (or differentiable) functions w.r.t. the supremum norm:

Definition 3.22. Let $S$ be some quasi-surface and $\sigma_{S}$ some intersection function for $S$.

Now let $\Lambda^{p, S, \sigma_{S}}$ for $p \in \mathbb{N} \cup\{\infty, \omega\}$ contain precisely all mappings

$$
\begin{aligned}
w^{S, \sigma_{S}}: C^{p}(M, \mathbf{G}) & \longrightarrow \mathcal{W}, \\
d & \longmapsto w_{d}^{S, \sigma_{S}}
\end{aligned}
$$

where $C^{p}(M, \mathbf{G})$ is equipped with the supremum norm on $S$.

We now may transfer this result to one-parameter subgroups. Using the one-parameter subgroups on $\mathbf{G}$ induced by the elements of the Lie algebra $\mathfrak{g}$, we have

Corollary 3.29. Let $\mathfrak{d}: M \longrightarrow \mathfrak{g}$ be a (not necessarily continuous) function, and define

$$
\begin{aligned}
E_{\mathfrak{d}}: \mathbb{R} & \longrightarrow \operatorname{Maps}(M, \mathbf{G}) . \\
t & \longmapsto\left(\mathrm{e}^{t \mathfrak{d}(x)}\right)_{x \in M}
\end{aligned}
$$

Then we have:

1. $E_{\mathfrak{d}}\left(t_{1}\right) E_{\mathfrak{d}}\left(t_{2}\right)=E_{\mathfrak{d}}\left(t_{1}+t_{2}\right)$ for all $t_{1}, t_{2} \in \mathbb{R}$.

2. $\mathrm{pr}_{x} \circ E_{\mathfrak{d}}$ is continuous for every $x \in M$.

3. The one-parameter subgroup

$$
t \longmapsto w_{E_{\mathfrak{d}}(t)}^{S, \sigma_{S}}
$$

is strongly continuous w.r.t. to $L_{2}\left(\overline{\mathcal{A}}, \mu_{0}\right)$ for each quasi-surface $S$ with intersection function $\sigma_{S}$.

Proof. The first two assertions are trivial. To see the strong continuity, apply Proposition 3.28 to the case $\Lambda_{0}:=\left\{E_{\mathfrak{d}}: \mathbb{R} \longrightarrow \operatorname{Maps}(M, \mathbf{G})\right\}$.

3.6. Graphomorphisms. One of the particular features of quantum geometry is its invariance w.r.t. diffeomorphisms of $M$. More precisely, diffeomorphisms act naturally on the paths inducing a $\mu_{0}$-invariant action on $\overline{\mathcal{A}}$ and, consequently, a unitary action on $\mathfrak{H}_{\text {aux }}$. The question remains, what kind of diffeomorphisms are to be admitted: analytic, piecewise analytic, smooth or something else? Anyway, we will postpone this discussion to Sect. 4 and consider here only some sort of minimal requirements. For this, let us again fix some smoothness class for the manifold and the paths in it.

Definition 3.23. A map $\varphi: M \longrightarrow M$ is called graphomorphism iff $\varphi$ is bijective and induces a groupoid isomorphism on $\mathcal{P}$. [13] 
Here, $\varphi(\gamma):=\varphi \circ \gamma$. Graphomorphisms have a convenient characterization [13]:

Lemma 3.30. A bijection $\varphi$ on $M$ is a graphomorphism iff $\varphi$ and $\varphi^{-1}$ map edges to $\mathcal{P}_{\text {gen }}$.

The action of graphomorphisms on $\mathcal{P}$ can be lifted to an action on $\overline{\mathcal{A}}$. In fact, each graphomorphism $\varphi$ defines via

$$
[\varphi(\bar{A})](\gamma):=h_{\bar{A}}\left(\varphi^{-1} \circ \gamma\right) \quad \text { for all } \gamma \in \mathcal{P}
$$

a map from $\overline{\mathcal{A}}$ to $\operatorname{Maps}(\mathcal{P}, \mathbf{G})$, again denoted by $\varphi$. We have

Proposition 3.31. Every graphomorphism $\varphi$ maps $\overline{\mathcal{A}}$ homeomorphically to $\overline{\mathcal{A}}$.

Proof. Of course, $\varphi$ maps $\overline{\mathcal{A}}$ to $\overline{\mathcal{A}}$. Moreover, $\pi_{\gamma} \circ \varphi=\pi_{\varphi^{-1} \circ \gamma}$ is continuous for all $\gamma \in \mathcal{P}$. Hence, $\varphi$ is continuous. The proof now follows, since $\varphi \circ \varphi^{-1}$ is the identity on $\overline{\mathcal{A}}$.

Proposition 3.32. The Ashtekar-Lewandowski measure $\mu_{0}$ is $\varphi$-invariant for all graphomorphisms $\varphi$.

Proof. This follows, because for all hyphs $v$,

$$
\left(\pi_{v}\right)_{*}\left(\varphi_{*} \mu_{0}\right)=\left(\pi_{\varphi^{-1} \circ v}\right)_{*} \mu_{0}=\mu_{\mathrm{Haar}}^{\#\left(\varphi^{-1} \circ v\right)}=\mu_{\mathrm{Haar}}^{\# v}=\left(\pi_{v}\right)_{*} \mu_{0} .
$$

Definition 3.24. For each graphomorphism $\varphi$ define $\alpha_{\varphi}$ to be the pull-back of $\varphi^{-1}$.

Proposition 3.33. For every graphomorphism $\varphi$,

- $\alpha_{\varphi}$ is an isometry on $C(\overline{\mathcal{A}})$;

- $\alpha_{\varphi}$ is a unitary operator on $L_{2}\left(\overline{\mathcal{A}}, \mu_{0}\right)$.

The map $\varphi \longmapsto \alpha_{\varphi}$ is even a representation of the group of graphomorphisms on $L_{2}\left(\overline{\mathcal{A}}, \mu_{0}\right)$, because $\alpha_{\varphi_{1} \circ \varphi_{2}}=\alpha_{\varphi_{1}} \circ \alpha_{\varphi_{2}}$ and $\alpha_{\varphi^{-1}}=\alpha_{\varphi}^{-1}$. $^{11}$

Graphomorphisms do not only act on graphs, but also on quasi-surfaces, intersection and other functions.

Definition 3.25. Let $\varphi$ be a graphomorphism. Then we set:

- $\varphi(S):=\varphi \circ S$ for every quasi-surface $S$;

- $\varphi(d):=d \circ \varphi^{-1}$ for every function $d: M \longrightarrow \mathbf{G}$;

- $[\varphi(\sigma)](S, \gamma):=\sigma\left(\varphi^{-1}(S), \varphi^{-1}(\gamma)\right)$ for every intersection function $\sigma$.

We, therefore, will have to guarantee that admissible homeomorphisms do not only preserve the set of paths under consideration, but also that of quasi-surfaces, and have to avoid ill-defined intersection functions - in particular, if we aim at an "intrinsic" assignment of intersection functions to quasi-surfaces. All that will be provided by using stratified analytic isomorphisms as to be discussed below.

Directly from the definitions, we get finally

Proposition 3.34. Let $\varphi: M \longrightarrow M$ be a graphomorphism, $S$ a quasi-surface, $\sigma$ an intersection function and $d: M \longrightarrow \mathbf{G}$ a function. Then we have

$$
w_{\varphi(d)}^{\varphi(S), \varphi(\sigma)}=\alpha_{\varphi}\left(w_{d}^{S, \sigma}\right) \equiv \alpha_{\varphi} \circ w_{d}^{S, \sigma} \circ \alpha_{\varphi}^{-1} .
$$

\footnotetext{
11 Note that we did not care about the corresponding covariance property for the Weyl operators. In fact, there $w$ is given by the pull-back of $\Theta$, not of $\Theta^{-1}$. Since, however, the $\Theta$-transforms do not form a group, that does not matter.
} 
3.7. Generalized gauge transforms. Any gauge theory incorporates gauge invariance. Therefore, we close this section with a few remarks on gauge transformations and, more general, bundle automorphisms.

Definition 3.26. The elements of $\overline{\mathcal{G}}:=\operatorname{Maps}(M, \mathbf{G})$ are called generalized gauge transforms. $^{12} \overline{\mathcal{G}}$ is given the product topology inherited from the canonical isomorphism $\operatorname{Maps}(M, \mathbf{G}) \cong \mathbf{G}^{M}$ and its group structure is given by pointwise multiplication.

Proposition 3.35. $\overline{\mathcal{G}}$ is a topological group and acts continuously on $\overline{\mathcal{A}}$ via

$$
h_{\bar{A} \circ \bar{g}}(\gamma):=\bar{g}(\gamma(0))^{-1} h_{\bar{A}}(\gamma) \bar{g}(\gamma(1)) \quad \text { for all } \gamma \in \mathcal{P} .
$$

Proposition 3.36. The Ashtekar-Lewandowski measure $\mu_{0}$ is invariant w.r.t. all generalized gauge transforms.

Definition 3.27. For each generalized gauge transform $\bar{g}$ define $\beta_{\bar{g}}$ on functions on $\overline{\mathcal{A}}$ by

$$
\left(\beta_{\bar{g}} f\right)(\bar{A}):=f(\bar{A} \circ \bar{g}) .
$$

Observe that $\beta_{\bar{g}_{1} \circ \bar{g}_{2}}=\beta_{\bar{g}_{1}} \circ \beta_{\bar{g}_{2}}$ and $\beta_{\bar{g}^{-1}}=\beta_{\bar{g}}^{-1}$.

Proposition 3.37. • $\bar{g} \longmapsto \beta_{\bar{g}}$ is a representation of $\overline{\mathcal{G}}$ on $C(\overline{\mathcal{A}})$ by isometries.

- $\bar{g} \longmapsto \beta_{\bar{g}}$ is a representation of $\overline{\mathcal{G}}$ on $L_{2}\left(\overline{\mathcal{A}}, \mu_{0}\right)$ by unitaries.

Generalized gauge transforms do also act on the G-valued functions labelling the quasi-surfaces.

Definition 3.28. Let $\bar{g}$ be a generalized gauge transform. Then we set:

- $\bar{g}(d):=\bar{g} \cdot d \cdot \bar{g}^{-1}$ for every function $d: M \longrightarrow \mathbf{G}$.

Again, directly from the definitions, we get

Proposition 3.38. Let $\bar{g}: M \longrightarrow M$ be a generalized gauge transform, $S$ a quasisurface, $\sigma$ an intersection function and $d: M \longrightarrow \mathbf{G}$ a function. Then we have

$$
w_{\bar{g}(d)}^{S, \sigma}=\beta_{\bar{g}}\left(w_{d}^{S, \sigma}\right) \equiv \beta_{\bar{g}} \circ w_{d}^{S, \sigma} \circ \beta_{\bar{g}}^{-1}
$$

3.8. Bundle automorphisms. Up to now, we have widely ignored the bundle structure of the gauge theory. Without a real need, we tacitly assumed to deal with a trivialized bundle, as we focused on the manifold $M$ and the structure group $\mathbf{G}$ only. Of course, it made the notations simpler and can, moreover, be justified a posteriori: $\overline{\mathcal{A}}$ contains the $C^{p}$ connections of any $\mathbf{G}$-principal bundle over $M$, independently from the bundle we started from. Similarly, $\overline{\mathcal{G}}$ contains all $C^{p}$ gauge transforms in any such bundle. But, conceptually, it is much more desirable to include the full bundle structure. Then we would also like to include the full group of bundle automorphisms. Note, here, that given any bundle automorphism $\theta: P \longrightarrow P$ of the G-bundle $P$ over $M$, we may extract from it a diffeomorphism $\varphi_{\theta}: M \longrightarrow M$ via

$$
\varphi_{\theta} \circ \operatorname{pr}_{M}=\operatorname{pr}_{M} \circ \theta,
$$

\footnotetext{
12 Starting from Sect. 4, we will usually drop the word "generalized" for simplicity.
} 
where $\mathrm{pr}_{M}$ denotes the canonical projection $\mathrm{pr}_{M}: P \longrightarrow M$. Moreover, the (smooth) gauge transforms correspond to vertical automorphisms; these are the bundle automorphisms with $\varphi_{\theta}=\mathrm{id}_{M}$.

Nevertheless, the full information on any (possibly stratified) $C^{p}$ bundle automorphism can be encoded in a (again, possibly stratified) $C^{p}$ diffeomorphism and a generalized gauge transform (even of any other bundle). The only danger arising from taking all the generalized gauge transforms of Subsect. 3.7 is to take too many gauge transforms. However, observe that, at least for the piecewise analytic category, the set of gauge orbits $\mathcal{A} / \mathcal{G}$ is densely embedded into $\overline{\mathcal{A}} / \overline{\mathcal{G}}$ and no two piecewise analytic connections fall into the same equivalence class by moding out the group of gauge transforms [18].

Finally, as it will turn out, the diffeomorphisms and the gauge transforms will play different rôles in the following proofs. Therefore, to make the basic ideas clearer and to sometimes allow for relaxed assumptions in the assertions, we will refrain from considering the fully automorphism invariant treatment of the Weyl algebra. Thus, w.l.o.g., we may pragmatically consider the bundle-automorphism invariance given by implementing both diffeomorphism and gauge invariance. The translation into the fully invariant language has to be left to the interested reader.

\section{Weyl Algebra of Quantum Geometry}

4.1. Structure data. In what follows, we are going to apply the above definitions and results to quantum geometry. Usually, this means to use piecewise analytic paths $\gamma$ and oriented hypersurfaces $S$ in $M$, whereas the intersection functions encode whether $\gamma$ intersects $S$ transversally or not and how its direction is related to the orientation of $S$. Moreover, (piecewise) analytic diffeomorphisms act on these objects. However, is it obvious that we should consider precisely these ingredients?

Before we discuss this question, let us collect these assumptions to avoid cumbersome notation.

Definition 4.1. The structure data of the theory under consideration contain:

- a manifold $M$;

- a Lie group $\mathbf{G}$;

- a smoothness class used for the definition of the set $\mathcal{P}$ of paths in $M$,

- a subset $\mathcal{S}$ of the set of quasi-surfaces in $M$,

- for each $S \in \mathcal{S}$ a subset $\Sigma(S)$ of the set of intersection functions for $S$,

- for each $S \in \mathcal{S}$ a subset $\Delta(S)$ of the set of functions from $M$ to $\mathbf{G}$,

- a subset $\mathcal{E}$ of the set $\overline{\mathcal{G}}$ of gauge transforms acting covariantly on $\Delta$;

- a subset $\mathcal{D}$ of the set of graphomorphisms on $M$ that leave $\mathcal{S}$ invariant and act covariantly on $\Sigma$ and $\Delta$;

Indeed, at first glance, there seems to be an enormous freedom in choosing structure data of a theory. However, there are several antagonists in the game. For instance, if we would enlarge $\mathcal{P}$, we might have to reduce $\mathcal{S}$, simply because we have to guarantee that there are at most finitely many (genuine) intersections of paths and quasi-surfaces. In fact, this practically excludes the choice of the smooth category for the paths: There are even analytic submanifolds having an infinite number of isolated transversal intersections with smooth paths. Therefore, we are - from the mathematical, technical point of view - quite forced to admit at most (piecewise) analytic paths. This however reduces the number of graphomorphisms in $\varphi$. Namely, they have to map analytic paths to (piecewise) 
analytic ones. This would lead directly into conflicts, if general smooth diffeomorphisms were allowed. They have to be "analyticity preserving" - at least for one-dimensional submanifolds. There are indeed classes of homeomorphisms having this property: At first, of course, analytic diffeomorphisms fulfill this requirement. However, this will not be sufficient for two reasons: On the one hand, analyticity usually implies high nonlocality - a feature not desired in gravity for physical reasons. On the other hand, in the sequel, the proofs will, in general, crucially depend on the locality for technical reasons as we will see later. Thus, some sort of piecewise analytic diffeomorphisms are to be admitted. In a natural way, this leads to stratified diffeomorphisms, because they map semianalytic sets (disjoint unions of analytic submanifolds forming stratifications) into semianalytic sets.

Next, we have to take care of the intersection functions. Given some oriented submanifold, say, a hypersurface, we would like to use this orientation to define such a function. However, this might lead to problems again: Using piecewise analytic diffeomorphisms, it may happen that a surface (including its orientation) is kept invariant, but an originally transversally intersecting path may now be mapped to a tangential one. ${ }^{13}$ This would contradict the concept that the intersection function encodes the transversality properties of a surface and its orientation, i.e., is assigned naturally and uniquely to an oriented surface. Of course, in contrast to the previous arguments, this rather is a conceptual demand and not a technical one. Moreover, it can be overcome using a slightly more special kind of piecewise analytic diffeomorphisms, as we will see later.

Third, the selection of functions is to be discussed. Since we have argued that mostly analytic (or piecewise analytic) objects are to be used, we could restrict ourselves again to (piecewise) analytic functions (at least for the restrictions to the respective surface). However, although this is possible, we may consider more general classes. In particular, after decomposing a surface into several submanifolds, we may admit functions that are analytic only on these submanifolds, but do not satisfy any continuity condition at their "boundaries". In fact, assume, e.g., that we are given a 2-surface $S$ and divide it by a line $S_{0}$ into two pieces $S_{1}$ and $S_{2}$ plus $S_{0}$ (like the interior of a circle is divided by a diameter). We now want to label $S$ on each $S_{i}$ by some analytic function $d_{i}$. We may take the Weyl operator $w_{0}$ for $S$ and $d_{0}$, then $w_{j 0}$ for $S_{j}$ with $\left(d_{0}\right)^{-1}$, and, finally, $w_{j}$ for $S_{j}$ and $d_{j}(j=1,2)$. Now, $w \circ w_{10} \circ w_{20} \circ w_{1} \circ w_{2}$ is the Weyl operator for $S$ with a function whose restriction on each $S_{i}$ is $d_{i}$. We should remark that this way one may even define submanifolds with codimension 2 or larger to be (quasi-)surfaces. This, however, brings back the problem that the intersection function is not necessarily given directly by the orientation of the submanifold itself: the transversality between paths and such lower-dimensional submanifolds would, in general, be destroyed already by analytic diffeomorphisms. Thus, one should restrict oneself to hypersurfaces (or at least semianalytic sets of pure codimension 1) and control lower-dimensional surfaces by including labellings of hypersurfaces with functions $d$ that are nontrivial only on these "sub"-surfaces. Or, equivalently, one may give lower-dimensional surfaces orientations that are induced by hypersurfaces containing them. We will exploit this idea. Anyway, after all, it does not seem necessary to impose very strong smoothness restrictions on $\Delta(S)$ from the conceptual point of view. Nevertheless, as we will see, there will be some technical difficulties that lead to restrictions.

13 Let $M$ be $\mathbb{R}^{2}$ and divide $M$ by the two lines $x= \pm 1$ into three open parts and the two lines. Now define $\varphi$ on the open strip between these two lines by $\varphi(x, y):=\left(x, y+\sqrt{1-x^{2}}\right)$ and let $\varphi$ be the identity otherwise. Of course, $\varphi$ is continuous everywhere and an analytic diffeomorphism on each of these five parts. Nevertheless, the path $\gamma$ with $\gamma(t)=(t, 0)$ is transversal w.r.t. $x=1$, but $\varphi(\gamma)$ is tangent to it. 
To summarize, in what follows we will always assume to work with "nice" structure data having the following minimal properties:

Definition 4.2. The structure data are called nice iff

- $M$ is an at least two-dimensional analytic manifold;

- $\mathbf{G}$ is a nontrivial, connected compact Lie group;

- $\mathcal{P}$ consists of all piecewise analytic paths in $M$;

- $\mathcal{S}$ contains at most the stratified analytic sets in $M$;

- $\Sigma(S)$ contains at least the natural ${ }^{14}$ intersection functions of $S$;

- $\Delta(S)$ contains at least the constant functions on $M$;

- $\mathcal{E}$ contains at least the trivial gauge transform;

- $\mathcal{D}$ contains at most the stratified analytic diffeomorphisms in $M$.

The requirements regarding regularity will be discussed in Subsect. 4.3. The precise definitions of stratified objects will be given in Sect. 6. Note, that whether we consider closed manifolds only or include open ones, is not decided here. The remaining "finetuning" will be made if needed.

4.2. Weyl algebra. Assume we are working with some arbitrary, but fixed "consistent" structure data. We define

$$
\mathcal{W}:=\left\langle\bigcup_{S \in \mathcal{S}} \bigcup_{\sigma_{S} \in \Sigma(S)} \bigcup_{d \in \Delta(S)}\left\{w_{d}^{S, \sigma_{S}}\right\}\right\rangle
$$

and set

$$
\mathcal{W}^{\prime}:=\left\langle\bigcup_{\varphi \in \mathcal{D}}\left\{\alpha_{\varphi}\right\}\right\rangle
$$

and

$$
\mathcal{W}^{\prime \prime}:=\left\langle\bigcup_{\bar{g} \in \mathcal{E}}\{\beta \bar{g}\}\right\rangle
$$

Definition 4.3. The $C^{*}$-subalgebra $\mathfrak{A}:=\mathfrak{A}\left(\mathcal{W}, \mu_{0}\right)$ of $\mathcal{B}\left(L_{2}\left(\overline{\mathcal{A}}, \mu_{0}\right)\right)$, generated by $C(\overline{\mathcal{A}})$ and $\mathcal{W}$, is called Weyl algebra of quantum geometry.

Definition 4.4. • $\mathfrak{A}_{\text {Diff }}:=\mathfrak{A}\left(\mathcal{W} \cup \mathcal{W}^{\prime}, \mu_{0}\right)$ denotes the $C^{*}$-subalgebra of $\mathcal{B}\left(L_{2}\left(\overline{\mathcal{A}}, \mu_{0}\right)\right)$ generated by $\mathfrak{A}$ and $\mathcal{W}^{\prime}$.

- $\mathfrak{A}_{\text {Auto }}:=\mathfrak{A}\left(\mathcal{W} \cup \mathcal{W}^{\prime} \cup \mathcal{W}^{\prime \prime}, \mu_{0}\right)$ denotes the $C^{*}$-subalgebra of $\mathcal{B}\left(L_{2}\left(\overline{\mathcal{A}}, \mu_{0}\right)\right)$ generated by $\mathfrak{A}, \mathcal{W}^{\prime}$ and $\mathcal{W}^{\prime \prime}$.

Definition 4.5. Let $\pi^{\prime}$ be a representation of $\mathfrak{A}_{\text {Diff }}$ on some Hilbert space $\mathfrak{H}$.

\footnotetext{
14 In contrast to Definition 3.16, we consider an intersection function on $S$ with $\operatorname{codim}_{M} S \geq 2$ to be natural iff it is induced by an embedded hypersurface $S^{\prime}$ that is contained in $\mathcal{S}$, not just in $M$. Moreover, one can directly extend the definition of natural intersection functions to stratified sets, e.g., using triangulations. However, since, at the end, we are interested mostly in the orientation of genuine submanifolds (possibly with boundary) only, we do not consider this issue in this paper in detail. Thus, at the moment, the statement " $\Sigma(S)$ contains at least the natural intersection functions of $S$ " only refers to such submanifolds $S$.
} 
- $\psi \in \mathfrak{H}$ is called diffeomorphism invariant (w.r.t. $\pi^{\prime}$ ) iff $\pi^{\prime}\left(\alpha_{\varphi}\right) \psi=\psi$ for all $\varphi \in \mathcal{D}$.

- $\pi^{\prime}$ is called diffeomorphism invariant iff it has a diffeomorphism invariant vector.

Often we write "D-invariant" instead of "diffeomorphism invariant".

Analogously, we speak about $\mathcal{D}$-natural representations meaning $\mathcal{W}^{\prime}$-natural representations.

Definition 4.6. Let $\pi^{\prime \prime}$ be a representation of $\mathfrak{A}_{\text {Auto }}$ on some Hilbert space $\mathfrak{H}$.

- $\psi \in \mathfrak{H}$ is called automorphism invariant (w.r.t. $\left.\pi^{\prime \prime}\right)$ iff $\left.\pi^{\prime \prime}\right|_{\mathfrak{A}_{\text {Diff }}}$ is diffeomorphism invariant and $\pi^{\prime \prime}(\beta \bar{g}) \psi=\psi$ for all $\bar{g} \in \mathcal{E}$.

- $\pi^{\prime \prime}$ is called automorphism invariant iff it has an automorphism invariant vector.

Usually we write "D-E-invariant" instead of "automorphism invariant".

Definition 4.7. $\pi_{0}$ denotes the fundamental (i.e., identical) representation of $\mathfrak{A}$ on $L_{2}\left(\overline{\mathcal{A}}, \mu_{0}\right)$ (and, analogously, that of $\mathfrak{A}_{\text {Diff }}$ and $\mathfrak{A}_{\text {Auto }}$, respectively).

Since $\mathbf{1} \in L_{2}\left(\overline{\mathcal{A}}, \mu_{0}\right)$ is already cyclic for $C(X) \subseteq \mathfrak{A}$, and $\alpha_{\varphi}(\mathbf{1})$ equals $\mathbf{1}$ for all $\varphi \in \mathcal{D}$ as well as $\beta_{\bar{g}}(\mathbf{1})$ does for all $\bar{g} \in \mathcal{E}$, we have

Proposition 4.1. 1 is a cyclic, diffeomorphism and automorphism invariant vector for $\pi_{0}$.

The irreducibility of $\pi_{0}$ will be proven separately in Sect. 5 .

4.3. Regularity. One of our goals in this paper is a uniqueness proof for certain representations of $\mathfrak{A}$. However, we will only be able to do this for certain regularity conditions. It is now reasonable to presuppose as little of them as possible. In other words, $\mathcal{R}$ which encodes the one-parameter subgroups to be mapped to weakly continuous ones, should be chosen as small as possible. As we will see, it will be sufficient to include that all $t \longmapsto w_{t}=w_{d(t)}^{S, \sigma_{S}}$ with $d(t):=\mathrm{e}^{t \mathfrak{d}} \in \Delta(S)$ for constant $\mathfrak{d}: M \longrightarrow \mathfrak{g}$. Of course, more regularity, hence larger $\mathcal{R}$, will not reduce uniqueness, but may even lead to the case that there is no such regular representation at all. Therefore, we are faced with some maximality conditions as well. First of all, we may at most allow for those one-parameter subgroups that map to the Weyl operators given by the structure data. Typically such restrictions are induced by the functions $d$ at our disposal. For instance, let $\mathbf{G}, M$ and $S$ be not simply connected, allow $\Delta(S)$ to contain continuous functions only, and let $d: M \longrightarrow \mathbf{G}$ have nontrivial mapping degree. Then, in general, it is not possible to deform $d$ in $\Delta(S)$ continuously into the trivial function on $\mathbf{G}$. This shows that it need not be possible to connect any Weyl operator to the identity within the limits of the structure data. Of course, using non-continuous $d$, it is always possible: Choose at every point $x$ in $M$ some $\mathfrak{d}(x) \in \mathfrak{g}$ with $\mathrm{e}^{\mathfrak{d}(x)}=d(x)$ and define $w_{t}:=w_{E_{\mathfrak{d}}(t)}^{S, \sigma_{S}}$ for all $t$. But, moreover, even if we might find for each $t$ some allowed $d(t)$ with $d\left(t_{1}+t_{2}\right)=d\left(t_{1}\right) d\left(t_{2}\right)$, the corresponding maps $t \longmapsto(d(t))(x)$ need not be continuous at all. The reason behind this is that the functional equation $f(x+y)=f(x)+f(y)$ has non-continuous, "cloudy" solutions. Then the corresponding one-parameter subgroups of Weyl operators are no longer weakly continuous, as one immediately checks. Therefore, we should restrict ourselves indeed to the functions generated by the Lie algebra functions. We summarize these considerations in 
Definition 4.8. Let $\mathcal{S}$ contain some quasi-surfaces in $M$ and, for each $S \in \mathcal{S}$, let $\Sigma(S)$ contain intersection functions for $S$ and $\Delta(S)$ contain functions from $M$ to $\mathbf{G}$.

$A$ set $\mathcal{R}$ of one-parameter subgroups in the set of Weyl operators is called full-consistent with $\mathcal{S},\{\Sigma(S)\}$ and $\{\Delta(S)\}$ iff for every element $t \longmapsto w_{t}$ in $\mathcal{R}$ there is some function $\mathfrak{d}: M \longrightarrow \mathfrak{g}$ and some quasi-surface $S \in \mathcal{S}$ with intersection function $\sigma_{S} \in \Sigma(S)$, such that $d(t):=\mathrm{e}^{t \mathfrak{d}} \in \Delta(S)$ and $w_{t}=w_{d(t)}^{S, \sigma_{S}}$ for all $t$.

$\mathcal{R}$ is called consistent with $\mathcal{S},\{\Sigma(S)\}$ and $\{\Delta(S)\}$ iff $\mathcal{R}$ equals $\left\langle\mathcal{R}_{0}\right\rangle$ for some $\mathcal{R}_{0}$ being full-consistent with $\mathcal{S},\{\Sigma(S)\}$ and $\{\Delta(S)\}$.

After all, we enlarge the structure data above by some subset $\mathcal{R}$ of the set of oneparameter subgroups in $\mathcal{W}$.

Definition 4.9. The enlarged structure data are called nice iff the structure data are nice and

- $\mathcal{R}$ contains at most the one-parameter subgroups of Weyl operators consistent with $\mathcal{S},\{\Sigma(S)\},\{\Delta(S)\}$ and at least those consistent with $\mathcal{S},\{\Sigma(S)\}$ and the constant functions.

Using Corollary 3.29 and Proposition 3.28, we have for nice enlarged structure data

Proposition 4.2. 1. $\pi_{0}$ is regular w.r.t. $\mathcal{R}$.

2. $\pi_{0}$ is $\Lambda$-regular with $\Lambda$ given in Proposition 3.28.

In particular, $\pi_{0}$ is $\Lambda^{p, S, \sigma_{S}}$-regular for all $p \in \mathbb{N} \cup\{\infty, \omega\}, S \in \mathcal{S}$ and $\sigma_{S} \in \Sigma(S)$.

\section{Irreducibility}

In this section we are going to prove the irreducibility of $\mathfrak{A}$ for nice structure data. [15] Additionally, we assume that $\mathcal{S}$ contains at least the closed, oriented hypersurfaces of $M$. Since we do not need diffeomorphisms, there will be no restrictions for $\mathcal{D}$. Note that given the irreducibility of the Weyl algebra of quantum geometry for these structure data, we get it immediately for all larger structure data. In fact, since the Weyl algebra cannot shrink if the structure data get larger, the commutant of the Weyl algebra cannot get larger in this case. Since, however, we will see it is already trivial for the assumptions above, the enlarged Weyl algebra is again irreducible.

5.1. Nice intersections. In this subsection, properties of intersections between graphs and surfaces, together with their implications for certain scalar products are studied.

Definition 5.1. Let $\gamma$ be an edge and let $\gamma$ be a (possibly trivial) graph.

A surface $S$ is called $(\gamma, \gamma)$-nice iff

1. $S$ is naturally oriented;

2. $S$ and (the image of) $\gamma$ are disjoint; and

3. $\gamma$ intersects $S$ in precisely one interior point $x$ of $\gamma$ transversally, such that the orientation of $S$ coincides with the direction of $\gamma$.

In this case, $x$ is called puncture of $S$ and $(\gamma, \gamma)$.

Lemma 5.1. Let $\gamma$ be an edge and $\gamma$ be a (possibly empty) graph, such that $\gamma$ and (the edges in) $\gamma$ intersect at most at their end points.

Then for every interior point $x$ of $\gamma$, there is a $(\gamma, \gamma)$-nice hypersurface $S$ with corresponding puncture $x$. 
Note that it does not matter whether we restrict ourselves to the case of closed surfaces or to that of open ones.

Proof. If we admit open surfaces $S$, then the assertion is trivial, since we may always find some neighbourhood of $x$ disjoint to $\gamma$, where $\gamma$ is a straight line. Take for $S$ some sufficiently small hyperplane "orthogonal" to $\gamma$ and that contains $x$.

Let us, therefore, consider the case of closed surfaces. Roughly speaking, the problem here is that if $\gamma$ "enters" $S$ at some point, it has to "leave" it somewhere else. Thus, we have to ensure that at only one point this intersection is transversal. For that purpose, we consider some (real) analytic curve $c$ in $\mathbb{R}^{2}$ that has an inflection point, such that the corresponding tangent $t$ intersects $c$ in precisely one other point $y$ transversally. Such curves exist - take, e.g., an appropriate Cassini curve [39]. As in the case of open surfaces, consider now some neighbourhood of $x$ isomorphic to $\mathbb{R}^{n} \supseteq \mathbb{R}^{2}$ and disjoint with $\gamma$, such that $x$ is mapped to $y$ and such that (the image of) $\gamma$ coincides with $t$ in some sufficiently large neighbourhood of $y$. Let now $S$ be the rotational surface given by $c$ and, e.g., the $x^{1}$-axis in $\mathbb{R}^{2} \subseteq \mathbb{R}^{n}$. By construction, $S$ has the required properties. (If the direction of $\gamma$ and the orientation of $S$ at the puncture do not coincide, simply mirror $S$ at the hyperplane "orthogonal" to $\gamma$.)

Lemma 5.2. Let $\gamma$ be an edge and let $\gamma$ be some (possibly trivial) graph, such that $\gamma$ and the edges in $\gamma$ intersect each other at most at their end points. Moreover, let $S$, $S_{1}$ and $S_{2}$ be $(\gamma, \gamma)$-nice surfaces, such that the corresponding punctures are different. Finally, let $T$ be a gauge-variant spin network function of the form $T=\left(T_{\phi, \gamma}\right)_{n}^{m} \otimes T^{\prime}$ with $T^{\prime} \in \mathcal{M}_{\gamma}$.

Then we have

$$
\left\langle w_{g_{1}}^{S_{1}}(T), w_{g_{2}}^{S_{2}}(T)\right\rangle=\frac{\overline{\chi_{\phi}\left(g_{1}^{2}\right)} \chi_{\phi}\left(g_{2}^{2}\right)}{(\operatorname{dim} \phi)^{2}}
$$

for all $g_{1}, g_{2} \in \mathbf{G}$. Moreover, if $\phi$ is abelian $^{15}$, we have

$$
w_{g}^{S}(T)=\phi\left(g^{2}\right) T=\chi_{\phi}\left(g^{2}\right) T
$$

for all $g \in \mathbf{G}$.

Here, $w_{g}^{S}$ is a shorter notation for $w_{d_{g}}^{S, \sigma_{S}}$ with $\sigma_{S}$ given by the natural orientation of $S$ and with $d_{g}$ being the function on $M$ constantly equal $g \in \mathbf{G}$.

Proof. First of all, note that $w_{g}^{S}(T)=w_{g}^{S}\left(\left(T_{\phi, \gamma}\right)_{n}^{m}\right) \otimes T^{\prime}$ for all $g \in \mathbf{G}$ and for all $(\gamma, \gamma)$-nice $S$. Assume now that $t_{1}<t_{2}$, where $\gamma\left(t_{j}\right)$ is the intersection point of $S_{j}$ and $\gamma$. Decompose $\gamma$ into the three segments $\gamma_{1}, \gamma_{0}$ and $\gamma_{2}$ according to the parameter intervals $\left[0, t_{1}\right],\left[t_{1}, t_{2}\right]$ and $\left[t_{2}, 1\right]$, respectively. Then we have

$$
\left(T_{\phi, \gamma}\right)_{n}^{m}=\left(T_{\phi, \gamma_{1} \gamma_{0} \gamma_{2}}\right)_{n}^{m}=\frac{1}{\operatorname{dim} \phi}\left(T_{\phi, \gamma_{1}}\right)_{p}^{m} \otimes\left(T_{\phi, \gamma_{0}}\right)_{q}^{p} \otimes\left(T_{\phi, \gamma_{2}}\right)_{n}^{q}
$$

\footnotetext{
15 Recall that a representation is called abelian (or linear) iff its character $\chi_{\phi}: \mathbf{G} \longrightarrow \mathbb{C}$ is multiplicative, i.e., $\chi_{\phi}\left(g_{1}\right) \chi_{\phi}\left(g_{2}\right)=\chi_{\phi}\left(g_{1} g_{2}\right)$ for all $g_{1}, g_{2} \in \mathbf{G}$. An irreducible abelian representation of a connected compact group is necessarily one-dimensional, i.e., $\phi(g)=\chi_{\phi}(g) \mathbf{1}$ with $\left|\chi_{\phi}(g)\right|=1$ for all $g \in \mathbf{G}$. Moreover, every compact connected $\mathbf{G}$ equals $\left(\mathbf{G}_{\mathrm{ss}} \times \mathbf{G}_{\mathrm{ab}}\right) / \mathbf{N}$ for some semisimple $\mathbf{G}_{\mathrm{ss}}$, some torus $\mathbf{G}_{\mathrm{ab}}$ and some discrete $\mathbf{N}$ being central in $\mathbf{G}_{\mathrm{sS}} \times \mathbf{G}_{\mathrm{ab}}$. Hence, for every irreducible representation $\phi$ of $\mathbf{G}$ there are irreducible representations $\phi_{\mathrm{ss}}$ and $\phi_{\mathrm{ab}}$ of $\mathbf{G}_{\mathrm{ss}}$ and $\mathbf{G}_{\mathrm{ab}}$, respectively, such that $\phi \circ \pi=\phi_{\mathrm{ss}} \otimes \phi_{\mathrm{ab}}$ with $\pi: \mathbf{G}_{\mathrm{ss}} \times \mathbf{G}_{\mathrm{ab}} \longrightarrow \mathbf{G}$ being the canonical projection. Then $\phi$ is abelian iff $\phi_{\mathrm{Ss}}$ is trivial.
} 
Consequently,

$$
\begin{aligned}
w_{g_{1}}^{S_{1}}\left(\left(T_{\phi, \gamma}\right)_{n}^{m}\right) & =\frac{1}{\operatorname{dim} \phi}\left(T_{\phi, \gamma_{1}}\right)_{r_{1}}^{m} \phi\left(g_{1}\right)_{p}^{r_{1}} \otimes \phi\left(g_{1}\right)_{s_{1}}^{p}\left(T_{\phi, \gamma_{0}}\right)_{q}^{s_{1}} \otimes\left(T_{\phi, \gamma_{2}}\right)_{n}^{q} \\
& =\frac{1}{\operatorname{dim} \phi} \phi\left(g_{1}^{2}\right)_{s_{1}}^{r_{1}}\left(T_{\phi, \gamma_{1}}\right)_{r_{1}}^{m} \otimes\left(T_{\phi, \gamma_{0}}\right)_{q}^{s_{1}} \otimes\left(T_{\phi, \gamma_{2}}\right)_{n}^{q}
\end{aligned}
$$

and, analogously,

$$
w_{g_{2}}^{S_{2}}\left(\left(T_{\phi, \gamma}\right)_{n}^{m}\right)=\frac{1}{\operatorname{dim} \phi} \phi\left(g_{2}^{2}\right)_{s_{2}}^{r_{2}}\left(T_{\phi, \gamma_{1}}\right)_{p}^{m} \otimes\left(T_{\phi, \gamma_{0}}\right)_{r_{2}}^{p} \otimes\left(T_{\phi, \gamma_{2}}\right)_{n}^{s_{2}} .
$$

Since $\gamma_{1}, \gamma_{0}, \gamma_{2}$ and $\gamma$ are independent, we get

$$
\begin{aligned}
\left\langle w_{g_{1}}^{S_{1}} T, w_{g_{2}}^{S_{2}} T\right\rangle & \\
= & \left\langle w_{g_{1}}^{S_{1}}\left(\left(T_{\phi, \gamma}\right)_{n}^{m}\right), w_{g_{2}}^{S_{2}}\left(\left(T_{\phi, \gamma}\right)_{n}^{m}\right)\right\rangle \cdot\left\langle T^{\prime}, T^{\prime}\right\rangle \\
= & \frac{1}{(\operatorname{dim} \phi)^{2}} \overline{\phi\left(g_{1}^{2}\right)_{s_{1}}^{r_{1}}} \phi\left(g_{2}^{2}\right)_{s_{2}}^{r_{2}} . \\
& \cdot\left\langle\left(T_{\phi, \gamma_{1}}\right)_{r_{1}}^{m},\left(T_{\phi, \gamma_{1}}\right)_{p}^{m}\right\rangle\left\langle\left(T_{\phi, \gamma_{0}}\right)_{q}^{s_{1}},\left(T_{\phi, \gamma_{0}}\right)_{r_{2}}^{p}\right\rangle\left\langle\left(T_{\phi, \gamma_{2}}\right)_{n}^{q},\left(T_{\phi, \gamma_{2}}\right)_{n}^{s_{2}}\right\rangle \\
= & \frac{1}{(\operatorname{dim} \phi)^{2}} \overline{\phi\left(g_{1}^{2}\right)_{s_{1}}^{r_{1}}} \phi\left(g_{2}^{2}\right)_{s_{2}}^{r_{2}} \delta_{r_{1} p} \delta^{s_{1} p} \delta_{q r_{2}} \delta^{q s_{2}} \\
= & \frac{1}{(\operatorname{dim} \phi)^{2}} \overline{\operatorname{tr} \phi\left(g_{1}^{2}\right)} \operatorname{tr} \phi\left(g_{2}^{2}\right) .
\end{aligned}
$$

If $t_{1}>t_{2}$, the calculation is completely analogous.

The assertion $w_{g}^{S}(T)=\phi\left(g^{2}\right) T$ for abelian $\phi$ follows directly from the definition of $w_{g}^{S}$. Recall that every abelian representation is one-dimensional and maps $\mathbf{G}$ to $U(1) \mathbf{1}$.

\subsection{Irreducibility proof.}

Theorem 5.3. The Weyl algebra $\mathfrak{A}$ of quantum geometry is irreducible on $L_{2}\left(\overline{\mathcal{A}}, \mu_{0}\right)$.

Before proving the theorem, we set $L_{\infty}:=L_{\infty}\left(\overline{\mathcal{A}}, \mu_{0}\right)$ and $L_{2}:=L_{2}\left(\overline{\mathcal{A}}, \mu_{0}\right)$.

Proof. We are now going to prove the irreducibility of $\mathfrak{A}$ by verifying that the commutant of $\mathfrak{A}$ consists of scalars only [12].

Since $C(\overline{\mathcal{A}}) \subseteq \mathfrak{A}$, we have $\mathfrak{A}^{\prime} \subseteq C(\overline{\mathcal{A}})^{\prime}=L_{\infty}$ for the commutants [36]. Next, one checks immediately that $w(f) w(\psi)=w(f \psi)$ for all $w \in \mathcal{W}, f \in L_{\infty}$ and $\psi \in L_{2}$. In other words, $w(f) \circ w=w \circ f$ in $\mathcal{B}\left(L_{2}\right)$.

Let now $f \in \mathfrak{A}^{\prime} \subseteq L_{\infty}$. Then we have $f \circ w=w \circ f=w(f) \circ w$ for all $w \in \mathcal{W}$, hence $w(f)=f$ in $L_{\infty} \subseteq L_{2}$ by invertibility of $w$. Consider additionally some nontrivial gauge-variant spin network function $T$. It can be written as $T=\left(T_{\phi, \gamma}\right)_{n}^{m} \otimes T^{\prime}$ with nontrivial $\phi$, where $T^{\prime} \in \mathcal{M}_{\gamma}$ is a (possibly trivial) spin network function, such that $\gamma$ and the edges in $\gamma$ intersect at most at their end points. By $w(f)=f$ and $w^{*} \in \mathcal{W}$ for all $w \in \mathcal{W}$, we have $\langle T, f\rangle=\left\langle T, w^{*}(f)\right\rangle=\langle w(T), f\rangle$ and, therefore,

$$
\langle w(T), f\rangle=\langle T, f\rangle=\left\langle w^{\prime}(T), f\right\rangle
$$

for all $w, w^{\prime} \in \mathcal{W}$. 
1. Let $\phi$ be abelian.

Choose some $(\gamma, \gamma)$-nice surface $S$ by Lemma 5.1. Then we have $w_{g}^{S}(T)=\phi\left(g^{2}\right) T$ for all $g \in \mathbf{G}$, by Lemma 5.2. Consequently,

$$
\langle T, f\rangle=\left\langle w_{g}^{S}(T), f\right\rangle=\overline{\phi\left(g^{2}\right)}\langle T, f\rangle .
$$

Since $\phi$ is nontrivial, there is some $g \in \mathbf{G}$ with $\phi\left(g^{2}\right) \neq 1$. Hence, $\langle T, f\rangle=0$.

2. Let $\phi$ be nonabelian.

Since $\mathbf{G}$ is compact and connected, there is a square root for each element of $\mathbf{G}$. Moreover, by [20], each nonabelian irreducible character has a zero. Hence, there is a $g \in \mathbf{G}$ with $\chi_{\phi}\left(g^{2}\right)=0$.

Choose now, by Lemma 5.1, infinitely many $(\gamma, \gamma)$-nice surfaces $S_{i}$, whose punctures with $\gamma$ are mutually different. Then, by Lemma 5.2, we have

$$
\left\langle w_{g}^{S_{i}}(T), w_{g}^{S_{j}}(T)\right\rangle=\frac{\overline{\chi_{\phi}\left(g^{2}\right)} \chi_{\phi}\left(g^{2}\right)}{(\operatorname{dim} \phi)^{2}}=0
$$

for $i \neq j$, due to the choice of $g$. Since $w_{g}^{S_{i}}$ is unitary, $\left\{w_{g} S_{i}(T)\right\}$ is an orthonormal system. Using

$$
\left\langle w_{g}^{S_{i}}(T), f\right\rangle=\left\langle w_{g}^{S_{j}}(T), f\right\rangle
$$

for all $i, j$, this implies $\left\langle w_{g}^{S_{i}}(T), f\right\rangle=0$ and thus $\langle T, f\rangle=0$.

Altogether, we have proven $\langle T, f\rangle=0$ for all nontrivial gauge-variant spin network functions $T$. Therefore, $f \in \mathbb{C} \mathbf{1}$, hence $\mathfrak{A}^{\prime}=\mathbb{C} \mathbf{1}$.

Corollary 5.4. $\mathfrak{A}_{\text {Diff }}$ and $\mathfrak{A}_{\text {Auto }}$ are irreducible.

\section{Stratified Diffeomorphisms}

As we have mentioned in Sect. 4 and we will see in the proofs, analytic graphomorphisms will not always be sufficient for studying representations of $\mathfrak{A}$. A natural extension is stratified analytic isomorphisms. The theory of stratifications we will use here is motivated by [21]. The first definition will be quoted almost literally, however, that of stratified maps is slightly sharpened. Although we will later apply the whole framework to the analytic category, we assume at this point only that we have fixed some smoothness category $C^{p}$ with $p \in \mathbb{N}$ or $p=\infty$ or $p=\omega$.

Let $M$ and $N$ be $C^{p}$ manifolds.

Definition 6.1. Let $A$ be some subset in $M$.

- A stratification $\mathcal{M}$ of $M$ is a locally finite, disjoint decomposition of $M$ into connected embedded $C^{p}$ submanifolds $M_{i}$ of $M$ (the so-called strata), such that

$$
M_{i} \cap \partial M_{j} \neq \varnothing \Longrightarrow M_{i} \subseteq \partial M_{j} \quad \text { and } \quad \operatorname{dim} M_{i}<\operatorname{dim} M_{j}
$$

for all $M_{i}, M_{j} \in \mathcal{M}$.

- A stratification $\mathcal{M}$ of $M$ is called stratification of $A$ in $M$ iff $A$ is the union of certain elements in $\mathcal{M}$.

- A is a stratified set (w.r.t. M) iff there is a stratification of $A$ in $M$. 
Definition 6.2. Let $\mathcal{M}_{1}$ and $\mathcal{M}_{2}$ be two stratifications of some subset of $A$.

Then $\mathcal{M}_{1}$ is called finer than $\mathcal{M}_{2}$ iff each stratum in $\mathcal{M}_{2}$ is a union of strata in $\mathcal{M}_{1}$.

Definition 6.3. A map $f: M \longrightarrow N$ is called

- stratified map iff $f$ is continuous and there are stratifications $\mathcal{M}$ and $\mathcal{N}$ of $M$ and $N$, respectively, such that for every $M_{i} \in \mathcal{M}$ there is an open $U_{i} \subseteq M$ and a $C^{p}$ differentiable map $f_{i}: M_{i} \subseteq U_{i} \longrightarrow N$ with

$$
\overline{M_{i}} \subseteq U_{i},\left.\quad f_{i}\right|_{M_{i}}=\left.f\right|_{M_{i}}, \quad f_{i}\left(M_{i}\right) \in \mathcal{N},\left.\quad \operatorname{rank} f\right|_{M_{i}}=\operatorname{dim} f\left(M_{i}\right)
$$

- stratified monomorphism iff, additionally, $\left.f\right|_{M_{i}}$ is injective;

- stratified isomorphism ${ }^{16}$ iff, additionally, $f$ is a homeomorphism and each $f_{i}$ : $U_{i} \longrightarrow f_{i}\left(U_{i}\right)$ is a $C^{p}$ diffeomorphism.

If we drop the above conditions that $U_{i}$ is open and that $\bar{M}_{i}$ is contained in $U_{i}$, we speak about weakly stratified maps.

Definition 6.4. A stratified map $f: M \longrightarrow M$ is called localized iff $f$ is the identity outside some compact subset of $M$.

Definition 6.5. Two stratified sets $S_{1}$ and $S_{2}$ in $M$ are called (weakly) strata equivalent iff there is a product of localized (weakly) stratified isomorphisms mapping $S_{1}$ onto $S_{2}$. They are called oriented-strata equivalent iff there is such a product mapping additionally the orientation of $S_{1}$ to that of $S_{2}$.

6.1. Localized Stratified Diffeomorphisms in Linear Spaces. In the sections below, we will have to study the local transformation behaviour of geometric objects in manifolds. To get prepared for this, we will now investigate first the corresponding problems in linear spaces. In particular, we will be able to rotate, scale and translate these objects locally, i.e., by transformations that are the identity outside some bounded region. This guarantees that we may lift the corresponding operations to manifolds.

We recall that a $q$-simplex $S$ in $\mathbb{R}^{k}$ with $q \leq k$ is the closed convex hull of $q+1$ points in general position. The corresponding interior of $S$ is called open simplex. Moreover, the (open) faces of $S$ are the (open) simplices spanned by subsets of these $q+1$ points. Additionally, we denote by $B_{r}^{q}(x)$, or shortly $B_{r}(x)$, some closed $q$-dimensional ball in $\mathbb{R}^{k}$ with radius $r$ around $x$. If $x$ is the origin, we simply write $B_{r}$. We remark that, in this subsection, nice orientations of some simplex or ball $S$ will always mean an orientation induced by that of some hyperplane (i.e., not by some more general hypersurface as for natural orientations) containing $S$. This implies, e.g., that the nice orientation of a $q$-simplex $S$ is always induced by some $(k-1)$-simplex having $S$ as one of its faces.

Finally, let us remark that in most of the statements of this subsection we will use 0 as a base point. It should be clear that all these statements hold analogously if 0 is replaced by any point in $\mathbb{R}^{k}$.

16 Sometimes we will use "stratified diffeomorphism" synonymously. 


\subsubsection{Strata equivalence of star-shaped regions.}

Lemma 6.1. Let $k$ be a positive integer and let $U$ be an open subset of $\mathbb{R}^{k}$ not containing 0 . Next, let $a, b, p: U \longrightarrow \mathbb{R}$ be $C^{p}$-functions, such that both $a, p$ and $p a+b$ are positive on $U$. Moreover, for every $\lambda>0$, let

$$
\begin{aligned}
& p(\lambda x)=\lambda p(x), \\
& a(\lambda x)=a(x), \\
& b(\lambda x)=b(x),
\end{aligned}
$$

whenever both $\lambda x$ and $x$ are contained in $U$. Finally define $\rho, \rho_{\mathrm{inv}}: U \longrightarrow \mathbb{R}$ by

$$
\rho:=a+\frac{b}{p} \quad \text { and } \quad \rho_{\mathrm{inv}}:=\frac{1}{a}\left(1-\frac{b}{p}\right) .
$$

Then $\widehat{\rho}: U \longrightarrow \mathbb{R}^{k}$ defined by

$$
\widehat{\rho}(x):=\rho(x) x
$$

is a $C^{p}$ diffeomorphism between $U$ and $\widehat{\rho}(U)$ and maps (subintervals of) each half-ray $\mathbb{R}_{+} x$ into (subintervals of) the same half-ray. Moreover, its inverse is given by

$$
\widehat{\rho}^{-1}(x)=\rho_{\text {inv }}(x) x .
$$

Proof. $\widehat{\rho}$ is indeed $C^{p}$, since $p$ never vanishes. Since $\widehat{\rho}$ at a single $x$ is just a positive scalar multiplication, it maps (subintervals of) each half-ray $\mathbb{R}_{+} x$ into (subintervals of) the same half-ray. Moreover, $\rho$ is injective and the image of $\rho$ is an open subset of $\mathbb{R}^{k}$. Finally, one checks immediately that $\widehat{\rho}^{-1}$ is $C^{p}$ and that it is the inverse of $\rho$ by $p a+b>0$.

Lemma 6.2. Let $k$ be a positive integer. Let $S_{0}$ and $S_{1}$ be the boundaries of two bounded open regions $R_{0}$ and $R_{1}$ in $\mathbb{R}^{k}$ both containing 0 . Assume, moreover, that each $R_{i}$ is star-shaped, that the corresponding Minkowski functional $p_{i}$ for $R_{i}$ is $C^{p}$ and that each $S_{i}$ is an embedded $C^{p}$ submanifold of $\mathbb{R}^{k}$.

Then, for all real $\lambda_{ \pm}$and $\lambda_{0, \pm}$ with

$$
0<\lambda_{-}<\inf _{\mathbb{R}^{k} \backslash\{0\}} \frac{p_{1}}{p_{0}} \leq \lambda_{0,-} \quad \text { and } \quad \lambda_{0,+} \leq \sup _{\mathbb{R}^{k}\{\{0\}} \frac{p_{1}}{p_{0}}<\lambda_{+},
$$

there are $C^{p}$ mappings $\widehat{\rho}_{+}$and $\widehat{\rho}_{-}$with the following properties:

1. $\widehat{\rho}_{ \pm}$is a $C^{p}$ diffeomorphism from some open neighbourhood of $V_{ \pm}$onto some neighbourhood of $W_{ \pm}$. Here,

$$
\begin{aligned}
V_{-} & =\left\{x \in \mathbb{R}^{k} \mid \lambda_{-} \leq p_{1}(x) \text { and } p_{0}(x) \leq 1\right\}, \\
V_{+} & =\left\{x \in \mathbb{R}^{k} \mid 1 \leq p_{0}(x) \text { and } p_{1}(x) \leq \lambda_{+}\right\}, \\
W_{-} & =\left\{x \in \mathbb{R}^{k} \mid \lambda_{-} \leq p_{1}(x) \leq \lambda_{0,-}\right\} \\
W_{+} & =\left\{x \in \mathbb{R}^{k} \mid \lambda_{0_{+}} \leq p_{1}(x) \leq \lambda_{+}\right\}
\end{aligned}
$$

are compact sets with nonempty interior.

2. $\widehat{\rho}_{ \pm}$maps $S_{0}$ to $\lambda_{0, \pm} S_{1}$;

3. $\widehat{\rho}_{+}$and $\widehat{\rho}_{-}$coincide on $S_{0}$ if $\lambda_{0,-}=\lambda_{0,+}$; 
4. $\widehat{\rho}_{ \pm}$is the identity on $\lambda_{ \pm} S_{1}$;

5. $\widehat{\rho}_{ \pm}$maps subintervals of half-rays to subintervals of the same half-ray.

6. The restrictions of $\widehat{\rho}_{ \pm}$to (an appropriate open subset of) any linear subspace of $\mathbb{R}^{k}$ are diffeomorphisms into that linear subspace.

Corollary 6.3. Given the assumptions of Lemma 6.2, there is a stratified $C^{p}$ diffeomorphism $\varphi$ mapping $S_{0}$ to $\lambda_{0} S_{1}$ and $R_{0}$ to $\lambda_{0} R_{1}$ for some $\lambda_{-} \leq \lambda_{0} \leq \lambda_{+}$, such that $\varphi$ is the identity inside $\lambda_{-} R_{1}$ and outside $\lambda_{+} R_{1}$. Moreover, $\varphi$ can be chosen, such that it preserves half-rays and its restrictions to linear subspaces of $\mathbb{R}^{k}$ are stratified $C^{p}$ diffeomorphisms again.

Proof. Simply define $\varphi$ to equal $\widehat{\rho}_{ \pm}$on $V_{ \pm}$and to be the identity otherwise. Since these mappings coincide on the corresponding overlaps $\lambda_{-} S_{1}, S_{0}$ and $\lambda_{+} S_{1}$, we get the assertion.

Note that $\lambda_{ \pm}$does only depend on the relative shape of $S_{0}$ and $S_{1}$. In particular, $\lambda_{ \pm}$need not be changed if both $S_{0}$ and $S_{1}$ are scaled by the same factor.

Proof of Lemma 6.2. Denote $R_{ \pm}:=\lambda_{ \pm} R_{1}$ and, correspondingly, $S_{ \pm}:=\partial R_{ \pm} \equiv \lambda_{ \pm} S_{1}$. By choice of $\lambda_{ \pm}$, we have $\overline{R_{-}} \subseteq R_{0} \subseteq \overline{R_{0}} \subseteq R_{+}$. Furthermore, let us define $q:=\frac{p_{1}}{p_{0}}$ on $V:=\mathbb{R}^{k} \backslash\{0\}$ and let

$$
a_{ \pm}:=\frac{\lambda_{ \pm}-\lambda_{0, \pm}}{\lambda_{ \pm}-q} \quad \text { and } \quad b_{ \pm}:=\lambda_{ \pm} \frac{\lambda_{0, \pm}-q}{\lambda_{ \pm}-q}
$$

define functions $a_{ \pm}, b_{ \pm}: V \longrightarrow \mathbb{R}$. Of course, $a_{ \pm}$is positive. Since Minkowski functionals are semilinear ${ }^{17}$, we see immediately that $q$, and so $a$ and $b$ as well, are constant on each half-ray $\mathbb{R}_{+} x$. Observe that $a$ and $b$ are well defined by choice of $\lambda_{ \pm}$and $\lambda_{0, \pm}$. Finally, we define $\widehat{\rho}_{ \pm}(x):=\rho_{ \pm}(x) x$ on $V$ by

$$
\rho_{ \pm}:=a_{ \pm}+\frac{b_{ \pm}}{p_{1}}=\frac{\lambda_{ \pm}\left(p_{1}+\lambda_{0, \pm}-q\right)-\lambda_{0, \pm} p_{1}}{p_{1}\left(\lambda_{ \pm}-q\right)} .
$$

We have

$$
\begin{aligned}
\left(q-\lambda_{ \pm}\right)\left(p_{1} a_{ \pm}+b_{ \pm}\right) & =p_{1}\left(\lambda_{0, \pm}-\lambda_{ \pm}\right)+\lambda_{ \pm}\left(q-\lambda_{0, \pm}\right) \\
& =p_{1}\left(\lambda_{ \pm}\left(\frac{1}{p_{0}}-1\right)+\lambda_{0, \pm}\right)-\lambda_{ \pm} \lambda_{0, \pm} \\
& =\left(p_{1}-\lambda_{ \pm}\right) \lambda_{0, \pm}+p_{1} \lambda_{ \pm}\left(\frac{1}{p_{0}}-1\right)
\end{aligned}
$$

Let us check the properties of $\widehat{\rho}_{ \pm}$:

- $\widehat{\rho}_{ \pm}$is obviously a $C^{p}$ function mapping subintervals of half-rays to subintervals of the same half-ray.

- Let $x \in S_{0}$, i.e., $p_{0}(x)=1$, hence $q(x)=p_{1}(x)$. Then $p_{1}\left(\rho_{ \pm}(x) x\right)=\rho_{ \pm}(x) p_{1}(x)=$ $\lambda_{0, \pm}$, i.e., $\widehat{\rho}_{ \pm}(x) \in \lambda_{0, \pm} S_{1}$. In particular, $\widehat{\rho}_{-}(x)=\widehat{\rho}_{+}(x)$ if $\lambda_{0,-}=\lambda_{0,+}$.

- Let $x \in \lambda_{ \pm} S_{1}$, i.e., $p_{1}(x)=\lambda_{ \pm}$. Then $\rho_{ \pm}(x)=1$, hence $\widehat{\rho}(x)=x$.

17 This means $p(\lambda x)=\lambda p(x)$ for all $\lambda>0$. 
- Let $x \in V_{-}$, i.e., $p_{0}(x) \leq 1$ and $p_{1}(x) \geq \lambda_{-}$. From the lines above, we see that this implies $\left(p_{1} a_{-}+b_{-}\right)(x) \geq 0$, the equality holding iff $p_{0}(x)=1$ and $p_{1}(x)=\lambda_{-}$. This, however, is impossible, since $q(x)$ would be equal $\lambda_{-}<\inf _{V} q$. Therefore, $p_{1} a_{-}+b_{-}>0$ on $V_{-}$. Since, by construction, $V_{-}$is compact, there is some open neighbourhood of $V_{-}$where $p_{1} a_{-}+b_{-}>0$. Lemma 6.1 now shows that $\widehat{\rho}_{ \pm}$is a $C^{p}$ diffeomorphism on that neighbourhood. By the previous items we see that $\widehat{\rho}_{-}\left(V_{-}\right)=W_{-}$.

- The corresponding properties of $\widehat{\rho}_{+}$are proven completely analogously.

- By intersecting $R_{i}$ and $S_{i}$ with linear subspaces we get $C^{p}$ boundaries with $C^{p}$ Minkowski functionals again. The remaining statements are now clear.

6.1.2. Scaling. To study geometric objects in charts, it may be necessary to first shrink them to have enough "space". That this is (almost) always possible using stratified diffeomorphisms, guarantees the following

Lemma 6.4. Let $k$ be a positive integer and let $R$ be a bounded star-shaped open region in $\mathbb{R}^{k}$ containing 0 and having a $C^{p}$ differentiable Minkowski functional p. Moreover, assume that the boundary $S$ of $R$ is an embedded $C^{p}$ submanifold of $\mathbb{R}^{k}$.

Then for all $\lambda>0$ and all $\varepsilon \geq 0$, there is a stratified $C^{p}$ isomorphism $\varphi$ preserving half-rays, such that $\varphi=\lambda$ id on $\bar{R}$ and $\varphi=\mathrm{id}$ outside $(1+\varepsilon) \max (\lambda, 1) R$.

Proof. - Assume first $\lambda \geq 1$.

Choosing $\lambda_{0_{+}}:=\sqrt{\lambda}$ and $\lambda_{+}:=(1+\varepsilon) \sqrt{\lambda}$, we may apply Lemma 6.2 to $R_{0}:=R$ and $R_{1}:=\sqrt{\lambda} R$ with $p=p_{0}=\sqrt{\lambda} p_{1}$. For this, define

$$
\varphi(x):=\left\{\begin{array}{lll}
\lambda \text { id } & \text { on } & p^{-1}([0,1]) \\
\widehat{\rho}_{+} & \text {on } & p^{-1}([1,(1+\varepsilon) \lambda]) \\
\text { id } & \text { on } & p^{-1}([(1+\varepsilon) \lambda, \infty))
\end{array} .\right.
$$

Now, let $x \in S$, i.e., $p(x)=1$. Then, by construction, $p_{1}\left(\widehat{\rho}_{+}(x)\right)=\lambda_{0_{+}+}=\sqrt{\lambda}$, hence $p_{0}\left(\widehat{\rho}_{+}(x)\right)=\lambda=p_{0}(\lambda x)$. For $x \in(1+\varepsilon) \lambda S$, we have $p(x)=(1+\varepsilon) \lambda$, hence $p_{1}(x)=(1+\varepsilon) \sqrt{\lambda}=\lambda_{+}$. By definition, we get $\widehat{\rho}_{+}(x)=x$. Altogether, $\widehat{\rho}_{+}$ equals $\lambda$ id on $S=\partial R$ and id on $(1+\varepsilon) \lambda S=(1+\varepsilon) \lambda \partial R$. Therefore, $\varphi$ is a stratified diffeomorphism having the desired properties.

- Assume now $\lambda \leq 1$.

Define $\lambda_{+}:=\sqrt{1+\varepsilon}$ and $\lambda_{0,+}:=\lambda(\sqrt{1+\varepsilon})^{-1}$, and apply Lemma 6.2 to $R_{0}:=R$ and $R_{1}:=\sqrt{1+\varepsilon} R$ with $p=p_{0}=\sqrt{1+\varepsilon} p_{1}$ : Define

$$
\varphi(x):=\left\{\begin{array}{lll}
\lambda \text { id } & \text { on } & p^{-1}([0,1]) \\
\widehat{\rho}_{+} & \text {on } & p^{-1}([1,1+\varepsilon]) \\
\text { id } & \text { on } & p^{-1}([1+\varepsilon, \infty))
\end{array} .\right.
$$

For $x \in S$, i.e., $p(x)=1$, we have $p_{1}\left(\widehat{\rho}_{+}(x)\right)=\lambda_{0,+}=\lambda(\sqrt{1+\varepsilon})^{-1}$. Therefore, we get $p_{0}\left(\widehat{\rho}_{+}(x)\right)=\lambda=p_{0}(\lambda x)$. If, on the other hand, $x \in(1+\varepsilon) S$, we have $p(x)=1+\varepsilon$ and $p_{1}(x)=\sqrt{1+\varepsilon}=\lambda_{+}$, implying $\widehat{\rho}_{+}(x)=x$. Consequently, $\widehat{\rho}_{+}$ equals $\lambda$ id on $S=\partial R$ and id on $(1+\varepsilon) S=(1+\varepsilon) \partial R$. Now, $\varphi$ is a stratified diffeomorphism having the desired properties. 


\subsubsection{Rotation.}

Lemma 6.5. Let $k$ be a positive integer and let $r_{1}>r_{2}>0$ be real. Let $X \in \mathfrak{s o}(k)$, define $A:=\mathrm{e}^{X} \in S O(k)$ and denote the orthogonal projection from $\mathbb{R}^{k}$ to $(\operatorname{ker} X)^{\perp}$ by $P$.

Then there is a stratified diffeomorphism $\varphi$ of $\mathbb{R}^{k}$, such that

- $\varphi$ coincides with A on $B_{r_{2}}$;

- $\varphi$ is the identity outside of $B_{r_{1}}$;

- $\varphi$ is norm preserving;

- $\varphi$ is homotopic to the identity;

- $P \varphi=P$.

Proof. We stratify $\mathbb{R}^{k}$ into

$$
\text { int } \left.B_{r_{2}} \cup \partial B_{r_{2}} \cup \text { (int } B_{r_{1}} \backslash B_{r_{2}}\right) \cup \partial B_{r_{1}} \cup\left(\mathbb{R}^{k} \backslash B_{r_{1}}\right)
$$

and define three auxiliary $C^{p}$ functions $a_{i}: \mathbb{R} \longrightarrow \mathbb{R}$ with

$$
a_{12}(r):=1, \quad a_{234}(r):=\frac{r_{1}-r}{r_{1}-r_{2}} \quad \text { and } \quad a_{45}(r):=0 .
$$

One now immediately checks that

$$
\varphi(x):= \begin{cases}\mathrm{e}^{a_{12}(\|x\|) X} x & \text { if } x \in \text { int } B_{r_{2}} \cup \partial B_{r_{2}} \\ \mathrm{e}^{a_{234}(\|x\|) X} x & \text { if } x \in \partial B_{r_{2}} \cup\left(\text { int } B_{r_{1}} \backslash B_{r_{2}}\right) \cup \partial B_{r_{1}} \\ \mathrm{e}^{a_{45}(\|x\|) X} x & \text { if } x \in \partial B_{r_{1}} \cup\left(\mathbb{R}^{k} \backslash B_{r_{1}}\right)\end{cases}
$$

gives the desired map. ${ }^{18}$ For the homotopy property define $\varphi_{t}$ as above with $t X$ instead of $X$. Then $\varphi_{1}=\varphi$ and $\varphi_{0}=$ id.

Immediately from the proof, we get with the above assumptions:

Corollary 6.6. Let $k$ be a nonnegative integer and let $\varepsilon>0$. Moreover, let $\gamma_{\alpha}$ be the straight line in $\mathbb{R}^{2}$ connecting $(-\cos \alpha,-\sin \alpha)$ and $(\cos \alpha, \sin \alpha)$.

Then for each $\alpha \in \mathbb{R}$ there is a stratified isomorphism $\varphi_{\alpha}$ of $\mathbb{R}^{2} \oplus \mathbb{R}^{k}$, such that

- $\varphi_{\alpha}$ is the identity outside $B_{1+\varepsilon} \subseteq \mathbb{R}^{k+2}$;

- $\varphi_{\alpha}$ is norm preserving;

- $\varphi_{\alpha}$ is homotopic ${ }^{19}$ to the identity;

- $P \varphi_{\alpha}=P$, where $P$ is the canonical projection from $\mathbb{R}^{2} \oplus \mathbb{R}^{k}$ to $\mathbb{R}^{k}$;

- $\varphi_{\alpha}$ maps $\gamma_{0}$ to $\gamma_{\alpha}$.

Proof. Choose $X=\alpha\left(\begin{array}{rr}0 & 1 \\ -1 & 0\end{array}\right) \in \mathfrak{s o}(2) \subseteq \mathfrak{s o}(2) \times \mathfrak{s o}(k) \subseteq \mathfrak{s o}(2+k)$.

18 Moreover, note that the three functions used to define $\varphi$ are defined on full $\mathbb{R}^{k}$ (possibly, up to the origin).

19 The mapping is given by $t \longmapsto \varphi_{t \alpha}$. 


\subsubsection{Translation.}

Lemma 6.7. Let $k$ be some positive integer. Let $\gamma$ be some edge in $\mathbb{R}^{k}$ and $U$ be some neighbourhood of $\gamma$. Choose $r>0$, such that the balls with radius $r$ centered at $\gamma(0)$ and $\gamma(1)$, respectively, are contained in $U$.

Then there is a finite product of stratified $C^{p}$ diffeomorphisms of $\mathbb{R}^{k}$ being the identity outside $U$ and the translation by $\gamma(1)-\gamma(0)$ on $B_{r}(\gamma(0))$.

Proof. We only give the idea of the proof. The technical details are similar to that for the preceding statements. Moreover, in Lemma C.1 we will give a proof for a more specific type of translation.

Here, we cover $\gamma$ by (non-trivial) balls. By compactness, there is some $r^{\prime}$, such that finitely many balls with radius $r^{\prime}$ centered at points of $\gamma$ will cover $\gamma$ and such that the convex hull of "neighbouring" balls is contained in $U$. The idea now is to first shrink $B_{r}(\gamma(0))$ to $B_{r^{\prime}}(\gamma(0))$, then move parallelly this ball through the convex hulls of neighbouring balls and finally blow it up to its original size. All these operations are possible by the statements above without moving any point outside $U$.

6.1.5. Strata equivalence of simplices and balls. Let us now show that all $q$-simplices are not only isomorphic as simplices themselves, but can also be mapped into each other by localized stratified $C^{p}$ diffeomorphisms. Moreover, they are equivalent to $q$-dimensional balls.

Proposition 6.8. Let $q \leq k$ be two positive integers.

Then all $q$-simplices and all $q$-dimensional balls in $\mathbb{R}^{k}$ are strata equivalent.

For this, we first show that each $q$-simplex can be mapped to a $q$-dimensional ball.

Lemma 6.9. Let $q \leq k$ be two positive integers. Moreover, let $V:=\left\{v_{0}, \ldots, v_{q}\right\} \subseteq \mathbb{R}^{k}$ contain $q+1$ points in general position, such that 0 is contained in the interior of the $q$-simplex $R_{V}$ spanned by $V$. Finally, fix some $\varepsilon>0$ and some $r>0$, such that $R_{V}$ is contained completely in the interior of $B_{r}$.

Then there is a stratified $C^{p}$ diffeomorphism $\varphi$, being the identity outside of $B_{(1+\varepsilon) r}$, such that $R_{V}$ is mapped to $B_{r} \cap \operatorname{span}_{\mathbb{R}} V$.

Proof. Choose some set $V^{\prime}=\left\{v_{0}^{\prime}, \ldots, v_{k-q}^{\prime}\right\} \subseteq \mathbb{R}^{k}$ of $k-q+1$ points in general position, such that its span is complementary to that of $V$ and such that the $(k-q)$ simplex spanned by $W$ contains 0 in its interior and is contained in int $B_{r}$. Define for every $0 \leq i \leq q$ and $0 \leq j \leq k-q$ the set

$$
V_{i j}:=\{0\} \cup\left(V \backslash\left\{v_{i}\right\}\right) \cup\left(V \backslash\left\{v_{j}^{\prime}\right\}\right)
$$

now containing $k+1$ points in general position, hence each defining a $k$-simplex $R_{i j}$. These simplices form a complex, i.e., in particular, they share at most lower-dimensional faces. Let $R_{0}$ be the union of all these $(k-q+1)(q+1)$ simplices. Its boundary is the union of the simplices $V_{i j}^{0}$ spanned by $V_{i j} \backslash\{0\}$.

Let us now invoke Corollary 6.3. First of all, observe that the statement there can be extended directly to the case that $R_{0}$ is formed by a finite number of cones each having tip at 0 and each defined by $k$-simplices, such that these cones fill $\mathbb{R}^{k}$ completely and share at most the boundaries with each other. Of course, the requirements for $S_{0}$ have to be relaxed accordingly. We refrained from explicitly giving this form of 
Corollary 6.3 (and Lemma 6.2, respectively), since it would have made the proof even more technical without introducing new ideas. One simply has to construct the stratified diffeomorphism in the more general case for every cone (more precisely, some open appropriate neighbourhood of it) and then use that these mappings fit together at the boundaries. This however, follows from the coincidence of the Minkowski functionals at these boundaries, the construction of the maps in the proofs above and the invariance of half-rays.

Coming back to the present proof, define $R_{1}$ to be $B_{r}$. Then, by $R_{0} \subseteq B_{r}$, the corresponding Minkowski functionals fulfill $p_{1} \leq p_{0}$, and we may choose $\lambda_{+}=1+\varepsilon>$ 1. This means that, by Corollary 6.3, there is a stratified $C^{p}$ diffeomorphism $\varphi$ being the identity outside $\lambda_{+} B_{r}$, mapping $R_{0}$ to $R_{1}$ and $\partial R_{0}$ to $\partial R_{1}$. Now the assertion follows, since $\varphi$ preserves linear subspaces. Therefore, $R_{V}$ (being the intersection of $R_{0}$ with $\operatorname{span}_{\mathbb{R}} V$ ) is mapped to $B_{r} \cap \operatorname{span}_{\mathbb{R}} V$ being a $q$-dimensional ball.

Proof of Proposition 6.8. Let two $q$-simplices be given. Using Lemma 6.7, translate both, such that they contain 0 in their interior. Then each of them is strata equivalent to some $q$-dimensional sphere in $\mathbb{R}^{k}$, by Lemma 6.9. Shrinking these balls, if necessary, we make them of identical radius. Finally, by Lemma 6.5, we may find some localized stratified $C^{p}$ diffeomorphism rotating one ball into the other. Hence, these two $q$-simplices are strata equivalent to a (hence, any) $q$-dimensional ball.

Now we are going to mirror simplices and balls into each other.

Proposition 6.10. Let $q<k$ be two non-negative integers.

Then every $q$-simplex and every $q$-dimensional ball in $\mathbb{R}^{k}$ having a nice orientation, is strata equivalent to itself having inverse orientation.

Proof. First assume that $q=k-1$ and consider some $q$-dimensional ball $B$ around the origin. Choose $X \in \mathfrak{s o}(k)$, such that $X$ is zero on some $(k-2)$-dimensional linear subspace $V$ of $\operatorname{span}_{\mathbb{R}} B$ and generates a rotation in the two-dimensional complement in $\mathbb{R}^{k}$ spanned by the normal of $V$ in $\operatorname{span}_{\mathbb{R}} B$ and the normal of $\operatorname{span}_{\mathbb{R}} B$ in $\mathbb{R}^{k}$. In particular, it generates some map $A:=\mathrm{e}^{t X} \in S O(k)$, being minus the identity on this two-dimensional space for some $t$. Since only one of its "dimensions" belongs to $B$, the rotation $A$ inverts the orientation of $B$. Now, Lemma 6.5 guarantees the existence of some stratified diffeomorphism inverting the orientation of $B$.

To prove the statement for $q=k-1$ and a given $q$-simplex $S$, we map it to some $q$-dimensional ball $B$, invert its rotation and take the inverse of the first mapping to get $S$ back. Of course, the orientation of $S$ has been flipped.

Next, let $q$ be arbitrary and consider a $q$-simplex $S$. Since we work with nice orientations only, there is some $(k-1)$-simplex $S^{\prime}$ in $M$ having $S$ as one of its faces and inducing its orientation. Since we may invert the orientation of $S^{\prime}$, we also may invert that of $S$ by localized stratified diffeomorphisms.

To prove the remaining case of $q$-balls for arbitrary $q$, reuse the argumentation above for $q=k-1$ and reduce to the case of $q$-simplices.

Corollary 6.11. Let $q<k$ be two non-negative integers.

Then all $q$-simplices and all $q$-balls in $\mathbb{R}^{k}$ are oriented-strata equivalent, provided they have nice orientations.

Proof. Assume, first of all, that $S$ is a $q$-simplex or a $q$-ball containing the origin, and let $S$ be given two nice orientations. This means there are linear subspaces $T_{1}$ and $T_{2}$ 
inducing these orientations by their own nice ones. There is now some $A \in S O(k)$ leaving the $q$-plane spanned by $S$ invariant and mapping $T_{1}$ onto $T_{2}$. Hence $A$ maps the one orientation of $S$ to either the other one or the inverse of it. Hence, by Lemma 6.5, there is some localized stratified isomorphism mapping $S$ onto itself and transforming the orientations by $A$. By adding, if necessary, some localized stratified isomorphism inverting the orientation as given by Proposition 6.10, we get such a transformation mapping the two orientations of $S$ onto each other.

Let now $S_{i}$ be a $q$-simplex or a $q$-ball for $i=1,2$. Then we may map them by localized stratified diffeomorphisms to some $q$-simplex $S$ containing the origin. Since, as one checks immediately, these mappings can be chosen, such that the corresponding orientations of $S$ are nice ${ }^{20}$, there is a localized stratified diffeomorphism mapping one orientation of $S$ to the other, by the arguments above.

Without explicitly stating the proof, we have by arguments as in the proposition above:

Corollary 6.12. For every nicely oriented 1 -dimensional ball $S$ in $\mathbb{R}^{k}$ with $k \geq 3$, there are finitely many localized stratified isomorphisms, whose product is the identity on $S$, but inverts the orientation of $S$.

Finally, we are looking for objects that can be divided into two parts, such that the original one is, on the one hand, strata equivalent to both of them and, on the other hand, is the disjoint union of them. Moreover, the orientation should be preserved. For example, consider an open 2-simplex, i.e., a full open triangle. Intersecting it by a line through one corner and some point of the opposite edge, we get two triangles. If we take their interiors, then they are strata equivalent to the original triangle, however not a decomposition of it - simply the border line is missing. One the other hand, if we were taking it to just one of the subtriangles, then they are no longer strata equivalent. The solution of this problem is to consider at the beginning an open triangle plus be one of its open edges. Then, as above, we may divide the triangle by a line, now through some boundary point of the added edge. Now it is clear that the triangle plus edge is divided into twice a triangle plus edge and all three objects are strata equivalent. The generalization to higher dimensions is straightforward, but more technical:

Proposition 6.13. Let $q<k$ be two positive integers. Let $S$ be some open $q$-simplex in $\mathbb{R}^{k}$, and let $F$, one of its open $(q-1)$-faces. Finally, give $R:=S \cup F$ the orientation induced by one of the nice orientations of $\bar{S} \supseteq R$.

Then there are products $\varphi_{0}$ and $\varphi_{1}$ of localized stratified isomorphisms, such that $R$ is the disjoint union of $\varphi_{0} R$ and $\varphi_{1} R$ and the intersection function of $R$ is the joint intersection function of $\varphi_{0} R$ and $\varphi_{1} R$.

Proof. First of all, choose some open $(k-1)$-simplex $\widehat{S}$, such that its orientation is induced by one of the nice orientations of its closure which, on the other hand, induces the orientations of $S$ and $R$. Let $V$ be the set of $k$ points $\left\{v_{0}, \ldots, v_{k-1}\right\}$ in $\mathbb{R}^{k}$, such that

$$
\begin{aligned}
& \widehat{S} \text { is the interior of the simplex spanned by }\left\{v_{0}, \ldots, v_{k-1}\right\} \text {, } \\
& \widehat{F} \text { is the interior of the simplex spanned by }\left\{v_{1}, \ldots, v_{k-1}\right\}, \\
& S \text { is the interior of the simplex spanned by }\left\{v_{0}, \ldots, v_{q}\right\}, \\
& F \text { is the interior of the simplex spanned by }\left\{v_{1}, \ldots, v_{q}\right\} .
\end{aligned}
$$

20 One sees that all necessary transformations are locally "affine". 
Define $\widehat{R}:=\widehat{S} \cup \widehat{F} \cup S \cup F$. Now choose some $v$ in the open 1-face connecting $v_{0}$ and $v_{1}$, and cut $\widehat{R}$ by the plane spanned by $\left\{v, v_{2}, \ldots, v_{k-1}\right\}$ into two parts $\widehat{R}_{0}$ and $\widehat{R}_{1}$, whereas the intersection of this plane with $\widehat{R}$ is added to $\widehat{R}_{0}$, and $\widehat{R}_{1}$ is that "half" whose closure contains $v_{1}$. We now may decompose each $\widehat{R}_{i}$ into $\widehat{S}_{i} \cup \widehat{F}_{i} \cup S_{i} \cup F_{i}$, where

$$
\begin{aligned}
& \widehat{S}_{i} \text { is the interior of the simplex spanned by }\left\{v, v_{i}, v_{2}, \ldots, v_{k-1}\right\}, \\
& \widehat{F}_{i} \text { is the interior of the simplex spanned by }\left\{x_{i}, v_{2}, \ldots, v_{k-1}\right\}, \\
& S_{i} \text { is the interior of the simplex spanned by }\left\{v, v_{i}, v_{2}, \ldots, v_{q}\right\}, \\
& F_{i} \text { is the interior of the simplex spanned by }\left\{x_{i}, v_{2}, \ldots, v_{q}\right\}
\end{aligned}
$$

with $x_{0}=v$ and $x_{1}=v_{1}$. Obviously, $S \cup F=S_{0} \cup F_{0} \cup S_{1} \cup F_{1}$.

Let now $\varphi_{i}$ be products of localized stratified isomorphisms that leave $v_{j}$ with $j \geq 2$ and $v_{i}$ invariant, map $v_{1-i}$ to $v$ and map the simplex spanned by $\left\{v_{0}, \ldots, v_{k-1}\right\}$ onto that spanned by $\left\{\varphi_{i}\left(v_{0}\right), \varphi_{i}\left(v_{1}\right), v_{2}, \ldots, v_{k-1}\right\}$. It is easy to check that $\varphi_{i}(S \cup F)=S_{i} \cup F_{i}$ and that $\varphi_{i}$ may be chosen to have the desired orientation properties.

6.2. Localized stratified diffeomorphisms in manifolds. We are now going to transfer the results of the previous subsection to the case of general $C^{p}$ manifolds $M$.

Definition 6.6. A subset $S$ in $M$ is called (nicely oriented) $q$-simplex in the chart $(U, \kappa)$ iff $S \subseteq U$ is mapped by $\kappa$ to a $q$-simplex in $\mathbb{R}^{\operatorname{dim} M}$ (and the orientation of $S$ is induced by one of the natural orientations of some hyperplane in $\kappa(U)$ ).

Analogously, we may define $q$-balls. The definition of faces of $q$-simplices should be clear as well. We will speak about $q$-simplices and $q$-balls in general iff there is a chart of $M$, in which they are $q$-simplices or $q$-balls. Note that, at least locally, every simplex or ball $S$ having a natural orientation is nicely oriented, i.e., it is induced by some hypersurface being (an open set of) a hyperplane in some chart. In fact, let $N$ be some embedded submanifold in $M$ containing $S$ as an embedded submanifold and inducing its orientation. Then we may find some chart mapping $N$ locally into some hyperplane in the local chart image of $M$ and mapping $S$ locally into some plane in the local image of $N$.

Proposition 6.14. The statements of Propositions 6.8, 6.10 and 6.13 as well as of Corollaries 6.11 and 6.12 remain valid if we replace $\mathbb{R}^{k}$ by $M$ and assume all $q$-simplices and $q$-balls to be in one and the same connected chart and, moreover, nicely oriented there.

Proof. The only point to be shown is the case that the localized stratified isomorphism $\varphi$ needs more space in $\mathbb{R}^{\operatorname{dim} M}$ than provided by the chart denoted by $(U, \kappa)$. If this is the case, first shrink any occurring object $S$ (being a ball or a simplex) to a sufficiently small size. Indeed, since simplices and balls are assumed to be closed and the chart is open, $S$ - magnified (in the chart) by $1+\varepsilon$ w.r.t. some interior point - is again in $U$ for small $\varepsilon$. Therefore, the scaling lemma (Lemma 6.4) is applicable in order to shrink $S$ by any factor $\lambda \leq 1$. Now it may be necessary to move $S$ to some other place in $U$ inside this chart. To do this, we choose some path that $S$ is moved along. By compactness and continuity reasons, there is a finite number of open $k$-balls in $U$ covering this path. We now assume that $\lambda$ is chosen small enough that the accordingly shrunk $S$ can be transferred between any non-disjoint two of these balls by means of Lemma 6.7. This way it can be (parallelly) shifted between any two points in the chart. Using these ingredients of shrinking and shifting, it is now easy to generate the desired localized stratified isomorphisms by means of their counterparts in $\mathbb{R}^{\operatorname{dim} M}$. 
6.3. Application to the analytic category. Let us now come back to the analytic case, i.e., $p=\omega$. Recall [21] that a subset $A$ of an analytic manifold $M$ is called semianalytic iff $M$ can be covered by some open sets $U_{l}$, such that each $U_{l} \cap A$ is a union of connected components of a set $f_{1}^{-1}(0) \backslash f_{2}^{-1}(0)$, for $f_{1}$ and $f_{2}$ belonging to some finite family of real-valued functions analytic in $U_{l}$. Complements, finite intersections and finite unions of semianalytic sets are semianalytic again [10]. Moreover, it can be shown $[21,28]$ that every semianalytic set admits a semianalytic stratification, i.e., a stratification consisting of semianalytic strata only. ${ }^{21}$

Lemma 6.15. Let $\mathcal{M}_{1}$ and $\mathcal{M}_{2}$ be two stratifications of $M$.

Then there is a stratification $\mathcal{M}$ of $M$ being finer than $\mathcal{M}_{1}$ and $\mathcal{M}_{2}$.

Proof. For every semianalytic, hence stratifiable set $A$ in $M$ and every nonnegative integer $k$, we choose some semianalytic stratification $\mathcal{N}(A)$ of $A$ and let $\mathcal{N}_{k}(A)$ contain precisely the $k$-dimensional strata in $\mathcal{N}(A)$ contained in $A$. Moreover, let $n$ be the dimension of $M$.

Since the intersection of any two semianalytic sets is semianalytic, we may define

$$
\begin{aligned}
\mathcal{N}_{n, k} & :=\bigcup_{M_{1} \in \mathcal{M}_{1}, M_{2} \in \mathcal{M}_{2}} \mathcal{N}_{k}\left(M_{1} \cap M_{2}\right), \\
\mathcal{N}_{n}^{\prime} & :=\bigcup_{k<n} \mathcal{N}_{n, k}, \\
\mathcal{N}_{n} & :=\mathcal{N}_{n, n} .
\end{aligned}
$$

This means, $\mathcal{N}_{n, k}$ contains the $k$-dimensional strata given by all the intersections of elements in $\mathcal{M}_{1}$ and $\mathcal{M}_{2}$. Since the boundary of every semianalytic set is semianalytic again [29], hence stratifiable, we may define successively for decreasing $i$ :

$$
\begin{aligned}
\mathcal{N}_{i}^{\partial} & :=\bigcup_{N_{1}, N_{2} \in \mathcal{N}_{i+1}} \bigcup_{k} \mathcal{N}_{k}\left(\partial N_{1} \cap \partial N_{2}\right), \\
\mathcal{N}_{i, k} & :=\bigcup_{N \in \mathcal{N}_{i}^{\partial}} \bigcup_{N^{\prime} \in \mathcal{N}_{i+1}^{\prime}} \mathcal{N}_{k}\left(N \cap N^{\prime}\right), \\
\mathcal{N}_{i}^{\prime} & :=\bigcup_{k<i} \mathcal{N}_{i, k}, \\
\mathcal{N}_{i} & :=\mathcal{N}_{i, i} .
\end{aligned}
$$

Finally, we set

$$
\mathcal{M}:=\bigcup_{i=0}^{n} \mathcal{N}_{i}
$$

One immediately checks that $\mathcal{M}$ is a stratification. Moreover, by construction, it is finer than $\mathcal{M}_{1}$ and $\mathcal{M}_{2}$.

Corollary 6.16. Every (weakly) stratified isomorphism is a graphomorphism.

Proof. Let $\varphi$ be a weakly stratified isomorphism on $M$ mapping $\mathcal{M}_{1}$ to $\mathcal{M}_{2}$. Moreover, let $\gamma$ be some analytic edge. Since im $\gamma$ is a semianalytic set, there is some stratification $\mathcal{M}_{3}$ of im $\gamma$. Choosing some stratification $\mathcal{M}$ finer than $\mathcal{M}_{1}$ and $\mathcal{M}_{3}$, the image of $\gamma$ is a union of strata in $\mathcal{M}$; even a finite one, since $\operatorname{im} \gamma$ is compact. Refining $\mathcal{M}_{2}$ w.r.t.

\footnotetext{
21 When speaking about semianalytic sets in the following, we will tacitly assume that the corresponding stratifications are semianalytic.
} 
$\varphi$ and w.r.t. the refinement of $\mathcal{M}_{1}$ to $\mathcal{M}$, we see that $\varphi$ maps im $\gamma$ into a finite union of strata. This means that $\varphi(\gamma)$ is piecewise analytic. The assertion now follows from Lemma 3.30.

Corollary 6.17. The (weakly) stratified isomorphisms of $M$ form a subgroup of the group of homeomorphisms of $M$.

Proof. Of course, the inverse of a (weakly) stratified isomorphism is a (weakly) stratified isomorphism. Therefore, consider two (weakly) stratified isomorphisms $f_{1}$ and $f_{2}$, and choose some stratifications $\mathcal{M}_{11}, \mathcal{M}_{12}, \mathcal{M}_{21}$ and $\mathcal{M}_{22}$ of $M$, where $f_{i}$ maps $\mathcal{M}_{i 1}$ to $\mathcal{M}_{i 2}$. By the previous lemma, we may find some $\mathcal{M}$ refining $\mathcal{M}_{12}$ and $\mathcal{M}_{21}$. Using the isomorphy property of $f_{1}^{-1}$ and $f_{2}$, we refine $\mathcal{M}_{11}$ and $\mathcal{M}_{22}$ accordingly as well. With respect to these refined stratifications, $f_{1} \circ f_{2}$ is a (weakly) stratified isomorphism.

Corollary 6.18. The localized (weakly) stratified isomorphisms of $M$ form a subgroup of the group of homeomorphisms of $M$.

The deeper reason behind the investigation of simplices above is the fact that every manifold can be triangulized; this means, roughly speaking, it is isomorphic to some union of (open) simplices. Originally known for nonanalytic manifolds (see, e.g., $[37,38]$ ), this result has been extended later to semianalytic sets in analytic manifolds (see, e.g., [29]). Here, however, we need a notion somewhat stronger than the usual one. In fact, recall that all the results above on (closed) simplices require that they are contained in some chart in $M$. Therefore we first quote the definition of a triangulation from [29] (dropping, however, some condition) and then extend this notion to the case we need.

Definition 6.7. Let $\left\{M_{i}\right\}$ be a locally finite collection of semianalytic subsets of $M$.

- A triangulation of $\left\{M_{i}\right\}$ is a simplicial complex ${ }^{22} K$ together with a homeomorphism $f:|K| \longrightarrow M$, such that for every $\sigma \in K$,

1. $f(\sigma)$ is an embedded analytic submanifold of $M$;

2. $f_{\sigma}:=\left.f\right|_{\sigma}: \sigma \longrightarrow f(\sigma)$ is an analytic diffeomorphism;

3. $f(\sigma) \subseteq M_{i}$ or $f(\sigma) \subseteq M \backslash M_{i}$ for all $M_{i}$.

- A triangulation $(K, f)$ of $\left\{M_{i}\right\}$ is called wide iff for every $\sigma \in K$ there is some open chart in $M$ containing the closure of $f(\sigma)$ and mapping it to a simplex in that chart. If each $M_{i}$ is given a natural orientation, then we additionally require $f$ to map this orientation to a nice one on each of these simplices.

- $\left\{M_{i}\right\}$ is called (widely) triangulizable iff there is a (wide) triangulation of $\left\{M_{i}\right\}$.

Proposition 6.19. Every semianalytic set is triangulizable [29].

One immediately checks that (nicely oriented) $q$-balls and $q$-simplices are widely triangulizable. What remains unsolved is

Question 1. Is every semianalytic set widely triangulizable?

Until now, we did not find any proof for either answer in the literature ${ }^{23}$ nor are we able to decide it ourselves. There may be some hints for this answer to be affirmative. In

\footnotetext{
22 A simplicial complex is a locally finite collection $K$ of disjoint open simplices in some finite-dimensional linear space, such that each face of any simplex belongs to $K$ again. Moreover, $|K|$ denotes the union of all these simplices.

23 Nor were we able to find our definition in the literature.
} 
fact, as proven by Ferrarotti (cf. [31]), there is a so-called strong triangulation $(K, f)$ of any analytic submanifold $M$ of $\mathbb{R}^{n}$. This means that, firstly, for any $\sigma \in K$ there is some neighbourhood $U$ of $\sigma$ in the Euclidean space containing $K$, and some analytic $F_{\sigma}: U \longrightarrow \mathbb{R}^{n}$ with $F_{\sigma}=f_{\sigma}$ and, secondly, for every vertex $v$ in $K$, the derivative $\mathrm{d} f_{v}: \overline{\operatorname{St}(v, K)} \longrightarrow \mathbb{R}^{n}$ is injective. But, nevertheless, our case remains open.

6.4. Two types of localized stratified diffeomorphisms. In this subsection ${ }^{24}$ we will investigate in detail the types of stratified diffeomorphisms to be used for quantum geometry.

Firstly, we present a more elaborate version of winding diffeomorphisms introduced originally by Sahlmann [33]. The aim is to produce stratified diffeomorphisms that wind an edge such that it has a certain number of punctures at some given surface. This would be possible even in the analytic category if only the pure number of punctures would count and the precise parameter values of the edge at the punctures would not matter. In fact, then one can use the approximation theorems for smooth mappings by analytic ones [22]. If, however, the precise location of the punctures becomes relevant, then probably this is no longer sufficient. Therefore-nevertheless reusing the main idea by Sahlmann-we present here a more general statement in the stratified analytic category.

Secondly, we study how one can transform a given graph into a very large set of independent graphs, but minimally modifying other geometric objects. There will be two cases depending on whether a graph is contained in (the closure of) some surface or not. If not, we may leave the surface invariant pointwise. If, on the other hand, the graph is (partially) contained in the interior of the surface, then we may, at least, slightly transform the surface into itself getting an infinite number of different graphs. This, of course, is possible, only if this surface provided enough space, i.e., is at least two-dimensional.

\subsubsection{Winding diffeomorphisms.}

Proposition 6.20. Let $\operatorname{dim} M \geq 3$. Let $\boldsymbol{\gamma}$ be a graph, let $\gamma \in \boldsymbol{\gamma}$ be one of its edges and set $\boldsymbol{\gamma}^{\prime}:=\boldsymbol{\gamma} \backslash\{\gamma\}$. Moreover, let $G$ be a finite subset in $\mathbf{G}$.

Then there is

- some subinterval I $\subseteq[0,1]$,

- some nicely oriented, open, embedded, analytic hypersurface $S$, disjoint to im $\boldsymbol{\gamma}$, such that $S$ and $\mathrm{S}$ have a finite wide triangulation;

- some analytic function $d: S \longrightarrow \mathbf{G}$ and

- some $s \in \mathbb{Z}$,

such that for any sequences $\left(g_{j}\right) \subseteq G$ and $\left(\tau_{j}\right) \subseteq I$ (with $\tau_{j}<\tau_{j^{\prime}}$ for $j<j^{\prime}$ ) having even length, there is a stratified (analytic) diffeomorphism $\varphi$ with the following properties:

1. $S$ and $\operatorname{im} \varphi\left(\boldsymbol{\gamma}^{\prime}\right)$ are disjoint;

2. $d\left(\varphi\left(\gamma\left(\tau_{j}\right)\right)\right)=g_{j}$ for all $j$;

3. $\varphi(\gamma)$ intersects $S$ completely transversally;

\footnotetext{
24 Note that all results of this subsection remain true in arbitrary smoothness categories, provided one enlarges the definition of intersection functions a little bit. Actually, they are defined only for quasi-surfaces; but only in the analytic category, hypersurfaces are always quasi-surfaces. The reason for that was that not every $\gamma$ can be $S$-admissibly decomposed. Here, however, we might study the intersection behaviour of certain paths with $S$. This, of course, is possible in the general case of smoothness as well.
} 
4. $\left\{\varphi\left(\gamma\left(\tau_{j}\right)\right)\right\}_{j}$ is the set of $\varphi(\gamma)$-punctures of $S$;

5. $\sigma^{+}\left(S,\left.\varphi(\gamma)\right|_{\left[\tau_{j-1}, \tau_{j}\right]}\right)=(-1)^{j+s}=\sigma^{-}\left(S,\left.\varphi(\gamma)\right|_{\left[\tau_{j}, \tau_{j+1}\right]}\right)$ for all $j$.

Proof. First of all, since $\gamma$ is embedded into $M$, there is some neighbourhood of some subpath $\left.\gamma\right|_{I}$ of $\gamma$ and some cubic chart, such that $\gamma(I)$ is exactly the part of im $\gamma$ in that chart. Any diffeomorphism constructed below will be constant outside this chart. Therefore, to simplify notation, we may restrict ourselves to the case that $M$ is $\mathbb{R}^{n}$ coordinatized by $x \in \mathbb{R}, y \in \mathbb{R}$ and $\vec{z} \in \mathbb{R}^{n-2}$ (where $z$ is the first coordinate of $\vec{z}$ ) and that $\gamma(I)$ is the intersection of the chart and the $x$-axis. Next, for the surface $S$ we will choose some hypersurface $y=a$ parallel to the $x$-axis for some $a>0$. Here, $a$ is selected under the assumption that $y=2 a+2 \varepsilon_{0}$ and $y=-2 \varepsilon_{0}$ are still hypersurfaces in the chart for some $\varepsilon_{0}>0$ (of course, only that part of the hypersurfaces whose $x$ - and $\vec{z}$-values are admitted in the chosen cubic chart). Finally, choose some analytic $d: S \longrightarrow \mathbf{G}$ depending on $z$ only, such that every element of $G$ occurs somewhere (in a sufficiently close) neighbourhood of $z=0 .{ }^{25}$

Let now finitely many (mutually different) points $\tau_{j} \in I$ be given. The $\gamma$-images of these points will turn into the intersection points of the transformed $\gamma$ with $S$. Fix, additionally, some small $\varepsilon<\varepsilon_{0}$, such that the distance of any two of the marked points in $I$ is greater than $2 \varepsilon$ (and that, if necessary, each of the $\varepsilon$-neighbourhoods of the $\tau_{k}$ are both in $I$ and in the fixed chart).

Now, in the first step we move $\gamma$ the following way inside the $x$-y-plane: On the one hand, each segment of $\gamma$ outside the $\varepsilon$-neighbourhoods of the marked points is again parallel to the $x$-axis; now, however, alternately with $y=0$ and $y=2 a$. The $\varepsilon$-neighbourhoods, on the other hand, are the straight lines connecting these alternatingly lifted and unlifted segments. This way, the center of these neighbourhoods, i.e., the marked points themselves are mapped to half-way between the levels $y=0$ and $y=2 a$. In other words, precisely the marked points are the intersection points of the transformed $\gamma$ with $S$. Note, in particular, that this transformation of $\gamma$ can be done by a stratified isomorphism that does only change the $y$-coordinates of any point in $M$, but neither the $x$ - nor the $\vec{z}$-values (see Lemma C.1). Moreover, note that we have tacitly used that there is an even number of $\tau_{k}$ to end up at level $y=0$ again after moving the largest $\tau_{k}$. This finishes the first step.

We are now left with the problem to find the intersection points having the correct values of $d$ in the second step. Nicely, the idea of the first step can be used again. To see this, assume that $n=3$ and look at the scene from above the $(x, z)$-plane. Since we only changed the $y$-values of $\gamma$, no change can be seen from this perspective. Using our assignment of $d$ to $S$, we move the $\varepsilon$-neighbourhoods of the marked points of (the transformed) $\gamma$. Slightly more generally than in the first step, however, we let the "bumps" that these neighbourhoods are mapped to, return to the original line before this neighbourhood ends. More precisely, the segments outside these neighbourhoods are not shifted again, and the "bumps" map each $\tau_{k}$ to the correct "level" (i.e., $z$-coordinate) in order to get mapped to the point with the correct value $g_{k}$ of $d$. Note that here we only need to change the $z$-coordinates, but leave, in particular, the $y$-coordinates unchanged. This implies that the parameter values where $\gamma$ intersects $S$ after having been transformed by both steps, are precisely those of the $\gamma$ after the first transformation. If $n>3$, this step is completely analogous.

\footnotetext{
25 Such a function indeed exists: Choose some $b>0$, such that the surfaces defined by $y=a$ and $z=i b$ are contained in the chosen chart for all $i=1, \ldots, \# G$. Choose, additionally, for each $i$, some polynomial $p_{i}$ with $p_{i}(l)=\delta_{i l}$ and some $X_{i} \in \mathfrak{g}$ with $g_{i}=\mathrm{e}^{X_{i}}$. Now, define $d(x, y, \vec{z}):=\prod_{i} \mathrm{e}^{p_{i}\left(\frac{z}{b}\right) X_{i}}$. This function fulfills $d(x, y, i b)=g_{i}$ for all $i$.
} 


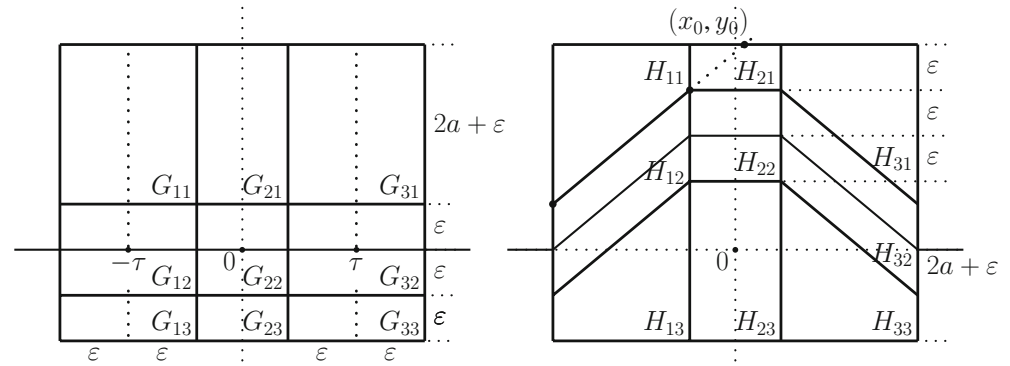

Fig. 1. Stratified Diffeomorphism in Lemma C.1

To summarize: It is clear that the constructed isomorphism has all the desired properties and that $s$ can be chosen obviously. Originally, we looked for a stratified isomorphism mapping $\gamma$, such that its transform intersects $S$ precisely for the parameter values $\tau_{k}$ and at points having the desired values of $d$. By the arguments above, we reduced this problem to the existence of a diffeomorphism in $\mathbb{R}^{n}$ as indicated in Fig. 1 that, in particular, does not move any point outside the given square (times some $\varepsilon$-ball in the remaining $n-2$ dimensions not drawn there). The existence of such a diffeomorphism, however, is guaranteed by Lemma C.1. This furnishes the present proof.

The crucial idea in the proof of Proposition 6.20 was to define for each element in $G$ some domain on the surface $S$, such that for a given sequence in $G$, the transformed graph punctures $S$ at the correct points and in the correct ordering, i.e., leading to the correct sequence of values for $d$. We constructed above a single surface with an analytic $d$ on it. However, we even might use constant $d$, if we admit $S$ to consist of more than one connected component. In other words, for any finite number we may find such a number of hypersurfaces $S_{i}$, such that $\gamma$ may always be transformed to puncture these different surfaces in an arbitrarily given ordering. More precisely, choose for $S_{i}$ some open (cubic) subspace in $S$, and let the only restriction to $S_{i}$ be that its $z$-coordinate is in some sufficiently small interval $I_{i}$. We may assume that the closures of these intervals are disjoint. Moreover, each $S_{i}$ is a hypersurface of $M$. Reusing the argumentation of the proof of Proposition 6.20, we have shown

Proposition 6.21. Let $\operatorname{dim} M \geq 3$. Let $\boldsymbol{\gamma}$ be a graph, let $\gamma \in \boldsymbol{\gamma}$ be one of its edges and set $\boldsymbol{\gamma}^{\prime}:=\boldsymbol{\gamma} \backslash\{\gamma\}$. Moreover, let $K$ be a positive integer.

Then there is

- some subinterval $I \subseteq[0,1]$,

- some nicely oriented, open, embedded analytic hypersurfaces $S_{i}$ with $i=1, \ldots, K$, such that each $S_{i}$ and each $\partial S_{i}$ has a finite wide triangulation, each $\overline{S_{i}}$ is disjoint to $\operatorname{im} \boldsymbol{\gamma}$, and all $\overline{S_{i}}$ are mutually disjoint; and

- some $s \in \mathbb{Z}$,

such that for any even integer $J>0$, any function $l:[1, J] \longrightarrow[1, K]$ and any sequence $\left(\tau_{j}\right) \subseteq I$ (with $\tau_{j}>\tau_{j^{\prime}}$ for $\left.j>j^{\prime}\right)$ having length $J$, there is a stratified analytic isomorphism $\varphi$ with the following properties:

1. $\bigcup_{i} \overline{S_{i}}$ and $\operatorname{im} \varphi\left(\boldsymbol{\gamma}^{\prime}\right)$ are disjoint;

2. $\varphi\left(\gamma\left(\tau_{j}\right)\right) \in S_{l(j)}$ for all $j$;

3. $\varphi(\gamma)$ intersects each $S_{i}$ completely transversally; 
4. $\left\{\varphi\left(\gamma\left(\tau_{j}\right)\right)\right\}_{j}$ is the set of $\varphi(\gamma)$-punctures of $\bigcup_{i} S_{i}$;

5. $\sigma^{+}\left(S_{l(j)},\left.\varphi(\gamma)\right|_{\left[\tau_{j-1}, \tau_{j}\right]}\right)=(-1)^{j+s}=\sigma^{-}\left(S_{l(j)},\left.\varphi(\gamma)\right|_{\left[\tau_{j}, \tau_{j+1}\right]}\right)$ for all $j$.

6.4.2. Generation of independent paths. Transferred to the case of manifolds, Corollary 6.6 yields

Proposition 6.22. Let $M$ be some $n$-dimensional manifold with $n \geq 2$ and let $S \subseteq M$. Assume that $S$ and $\partial S$ are connected embedded submanifolds in $M$ (without boundary) and that $\bar{S}$ is an embedded submanifold in $M$ having boundary $\partial S$. Moreover, let $\gamma$ be some nontrivial graph in $M$, such that the image of $\gamma$ is neither equal to $S$, a $S$ nor $\bar{S}$.

Then there is a nontrivial path $\gamma$, a neighbourhood $U$ of some $m \in \operatorname{im} \gamma$ in $M$ and infinitely many stratified diffeomorphisms $\varphi_{i}$ of $M$, such that

- $\gamma$ is the only edge in $\gamma$ not disjoint to $U$;

- $\varphi_{i}$ is the identity outside $U$;

- $\varphi_{i}$ leaves the set $S$ invariant;

- $\left\{\varphi_{i}(\gamma)\right\}_{i}$ is a hyph. ${ }^{26}$

If we additionally assume, that $S$ has one of its natural orientations, then each $\varphi_{i}$ may be chosen such that, additionally, it leaves the orientation of $S$ invariant.

Proof. 1. im $\gamma$ is not contained in $\bar{S}$.

Let $\gamma$ be an edge of $\gamma$ not contained in $\bar{S}$. Choose some interior point $m$ of $\gamma$ outside $\bar{S}$, and let $U$ be some open neighbourhood of $m$ disjoint to $S$ and disjoint to all other edges in $\gamma$ except for $\gamma$. Choose some chart whose closure is contained in $U$ and whose intersection with (the image of) $\gamma$ is mapped to a straight line with $m$ mapped to the origin. Corollary 6.6 now gives a collection $\varphi_{\alpha}$ of stratified diffeomorphisms being the identity outside the chart that, therefore, may be extended to stratified diffeomorphisms of $M$ that are the identity outside, at least, $U$. Since each $\varphi_{\alpha}(\gamma)$ with $\alpha \in[0, \pi)$ has some interior point not passed by any other $\varphi_{\alpha^{\prime}}(\gamma)$, these paths are independent. The invariance of $S$ is trivial as well as the fact that the orientation of $S$ is preserved and that $\left\{\varphi_{\alpha}(\gamma)\right\}_{\alpha}$ is a hyph.

2. im $\boldsymbol{\gamma}$ is contained in $\partial S$.

In particular, this implies that $\partial S$ is at least one-dimensional. In fact, otherwise $\partial S$ would be a point and $\gamma$ trivial. In the case that $\operatorname{dim} S<n-1$ and that we consider orientations, let, moreover, $S^{\prime} \supseteq S$ be some $(n-1)$-dimensional embedded submanifold of $M$ inducing the orientation of $S$.

a) $\operatorname{dim} \partial S \geq 2$.

Choose some interior point $m$ of some edge $\gamma$ in $\gamma$ and some open neighbourhood $U$ of $m$ whose closure is disjoint to all edges in $\gamma$ except for $\gamma$. By assumption, there is some chart whose closure is contained in $U$, such that the intersection of the chart

- with $S^{\prime}$ (if applicable) is some open subset of $\mathbb{R}^{n-1}$,

- with $S$ is some open subset of $\mathbb{R}^{\operatorname{dim} S}$ (if applicable, in $\mathbb{R}^{n-1}$ ),

- with $\partial S$ is some open subset of $\mathbb{R}^{\operatorname{dim} S-1} \subseteq \mathbb{R}^{\operatorname{dim} S}$,

- with im $\gamma$ is a straight line in $\mathbb{R} \subseteq \mathbb{R}^{\operatorname{dim} S-1}$, and

- with $m$ is mapped to the origin.

26 Here, we extended the notion of a hyph naturally to the case of infinitely many paths. 
Since $\operatorname{dim} S>\operatorname{dim} \partial S \geq 2$, Corollary 6.6 provides us, analogously to the first case, with stratified diffeomorphisms having the desired properties. In particular, observe that, although they are not the identity neither on $S$ nor on $\partial S$, they leave both $S$ and $\partial S$ (and, if applicable, $S^{\prime}$ ) invariant.

The orientation of $S$ is obviously preserved for $\operatorname{dim} S=n$. For $\operatorname{dim} S=n-1$, use the fact that $t \longmapsto \varphi_{t \alpha}$ is a homotopy over diffeomorphisms having the properties above, whence the natural orientation of $S$ is preserved by each diffeomorphism. If $\operatorname{dim} S<n-1$, then the natural orientations of $S^{\prime}$ are preserved as above, whence the induced orientations on $S$ are so as well.

b) $\operatorname{dim} \partial S=1$.

Since $\partial S$ is one-dimensional, it is isomorphic to either a line or a circle. Moreover, $\partial \partial S=\varnothing$. Since the compact set $\operatorname{im} \gamma$ does not equal $\partial S$, there is some point $m \in \partial(\operatorname{im} \gamma) \subseteq \partial S$. Moreover, there is a (unique) edge, say $\gamma$, having $m$ as one of its endpoints. We may assume $\gamma(0)=m$ and choose some open neighbourhood $U$ of $m$ whose closure is disjoint to all edges in $\boldsymbol{\gamma}$ except for $\gamma$. Now, we select some chart whose closure is contained in $U$, such that the intersection of the chart

- with $S^{\prime}$ is (if applicable) some open subset of $\mathbb{R}^{n-1}$,

- with $S$ is some open subset of $\mathbb{R}^{2}$ (if applicable, in $\mathbb{R}^{n-1}$ ),

- with $\partial S$ is some open subset of $\mathbb{R} \subseteq \mathbb{R}^{2}$,

- with im $\gamma$ equals $[0, \tau) \subseteq \mathbb{R}$, and

- with $m$ is mapped to the origin,

and such that $B_{\tau} \subseteq U$ for some $\tau>0$. By Lemma 6.4, for $\alpha \in\left[0, \frac{1}{3} \tau\right)$, there are now stratified diffeomorphisms $\varphi_{\alpha}$, taking $-\frac{1}{3} \tau \in \mathbb{R} \subseteq \mathbb{R}^{n}$ as the origin, such that $(-\tau,+\tau)$ is mapped onto itself, such that $\varphi_{\alpha}([0, \tau])=[\alpha, \tau]$ and such that $\varphi_{\alpha}$ is the identity outside $U$. In particular, each $\varphi_{\alpha}$ leaves both $S, \partial S$ and $S^{\prime}$ invariant. Choosing some monotonously decreasing, infinite sequence $\alpha_{i} \rightarrow 0$, we get a hyph $\left\{\varphi_{\alpha_{i}}(\gamma)\right\}_{i \in \mathbb{N}}$, since $\left(\alpha_{i}, \alpha_{i-1}\right)$ is passed by no $\varphi_{\alpha_{j}}(\gamma)$ with $j<i$. The preservation of orientation by $\varphi_{\alpha_{i}}$ is shown analogously to the case above.

3. im $\gamma$ is contained in $\bar{S}$, but not in $\partial S$.

As above, this implies that $S$ is at least one-dimensional. Again, for $\operatorname{dim} S<n-1$ and if we consider orientations, we let $S^{\prime} \supseteq S$ be some $(n-1)$-dimensional embedded submanifold of $M$ inducing the orientation of $S$.

a) $\operatorname{dim} S \geq 2$.

Choose in $\gamma$ some edge $\gamma$ not fully contained in $\partial S$. We now may find some interior point $m$ of $\gamma$ being in the interior of $S$ and fix some open neighbourhood $U$ of $m$, whose closure is disjoint to $\partial S$ and disjoint to all edges in $\gamma$ except for $\gamma$. By assumption, there is some chart whose closure is contained in $U$, such that the intersection of the chart

- with $S^{\prime}$ is (if applicable) some open subset of $\mathbb{R}^{n-1}$,

- with $S$ is some open subset of $\mathbb{R}^{\operatorname{dim} S}$ (if applicable, in $\mathbb{R}^{n-1}$ ),

- with im $\gamma$ is a straight line in $\mathbb{R} \subseteq \mathbb{R}^{\operatorname{dim} S}$, and

- with $m$ is mapped to the origin.

As above, we may find stratified diffeomorphisms of the desired type, by Corollary 6.6. 
b) $\operatorname{dim} S=1$.

Since $S$ is one-dimensional, it is isomorphic to either a line or a circle. Hence $\partial S$ consists of at most two points. Consequently, $\bar{S}$ is isomorphic either to a circle, a line, a ray or a closed interval. Since im $\gamma \subset \bar{S}$ is compact, there is some point $m \in \partial(\operatorname{im} \boldsymbol{\gamma}) \cap S$. Picking, as above, the (unique) edge $\gamma$ having $m$ as one of its endpoints, we now may find some open neighbourhood $U$ of $m$ whose closure is disjoint to $\partial S$ and to all edges in $\gamma$ except for $\gamma$. Again, we select some chart whose closure is contained in $U$, such that the intersection of the chart

- with $S^{\prime}$ is (if applicable) some open subset of $\mathbb{R}^{n-1}$,

- with $S$ is some open subset of $\mathbb{R}$ (if applicable, in $\mathbb{R}^{n-1}$ ),

- with im $\gamma$ equals $[0, \tau) \subseteq \mathbb{R}$, and

- with $m$ is mapped to the origin,

and such that $B_{\tau} \subseteq U$ for some $\tau>0$. Again, as in the case im $\gamma \subseteq \partial S$ and $\operatorname{dim} \partial S=1$, we find the desired stratified diffeomorphisms by Lemma 6.4.

Proposition 6.23. Let $M$ be some $n$-dimensional manifold with $n \geq 2$ and let $S \subseteq M$. Assume that $S$ and $\partial S$ are connected embedded submanifolds in $M$ (without boundary) and that $\bar{S}$ is an embedded submanifold in $M$ having boundary $\partial S$. Moreover, let either $S$ or $\partial S$ be an embedded 1-circle $S^{1}$. Finally, let $\gamma$ be a graph whose image is this $S^{1}$ and let $m$ be some vertex of $\gamma$.

Then there is a neighbourhood $U$ of $m$ in $M$, infinitely many different $m_{i}$ in $S^{1} \cap U$ and for each $i$ a stratified diffeomorphism $\varphi_{i}$ of $M$ with the following properties:

- $\varphi_{i}$ is the identity outside $U$;

- $\varphi_{i}$ leaves the set $S$ invariant;

- $\varphi_{i}$ maps $m$ to $m_{i}$;

- $\varphi_{i}$ is the identity on all edges of $\boldsymbol{\gamma}$ not adjacent to $\mathrm{m}$.

If we additionally assume that $S$ has one of its natural orientations, then each $\varphi_{m^{\prime}}$ may be chosen such that, additionally, it leaves the orientation of $S$ invariant.

Proof. This proof is very analogous to that of Proposition 6.23. Therefore, we only present its main idea.

First choose some $U$, small enough to intersect im $\gamma$ only at its edges adjacent to $m$ and such that $U \cap \bar{S}$ is a domain of a straight line (if im $\gamma=S$ ) or of a half plane (if im $\boldsymbol{\gamma}=\partial S$ ). Now choose some point near $m$ as the origin for a local scaling as in Lemma 6.4. This way, we may move $m$ to every other sufficiently near-by point, leaving $\partial S$ or $S$, respectively, invariant, without moving any point outside $U$.

\section{Representations of the Weyl Algebra}

Now we are prepared to give a rigorous proof of (a stronger version of the) uniqueness theorem claimed by Sahlmann and Thiemann [35]. As well, we will proceed in two steps: First we use regularity and diffeomorphism invariance to show that the first-step decomposition contains the Ashtekar-Lewandowski measure. This will follow from the fact that the diffeomorphisms split the Weyl operators, i.e., the weak convergence of Weyl operators is not uniformly on states related by diffeomorphisms. Second, using diffeomorphism invariance again, we show that each Weyl operator is a scalar at this component. This enables us to use the naturality of the action of diffeomorphisms in order to prove that each Weyl operator is even a unit there. Cyclicity will give the proof. At the end, we discuss the technical assumptions made in the proofs. 
7.1. Splitting property. As before, we assume to be given some nice enlarged structure data. Moreover, we restrict ourselves to the case that $\mathcal{S}$ and $\mathcal{D}$ contain at least those hypersurfaces and stratified isomorphisms, respectively, that are necessary to keep Proposition 6.21 valid. In other words, one possibility is to choose $\mathcal{S}$ to contain at least all of these "cubic" hypersurfaces and $\mathcal{D}$ to contain at least the stratified isomorphisms described in Subsubsect. 6.4.1. Throughout the whole subsection, let $\pi^{\prime}$ be some representation of $\mathfrak{A}_{\text {Diff }}$ on $\mathfrak{H}$ and denote by $\pi:=\left.\pi^{\prime}\right|_{\mathfrak{A}}$ the corresponding representation of $\mathfrak{A}$. Additionally, we require $M$ to have at least dimension 3 .

Proposition 7.1. Assume $\pi$ to be regular. Moreover, let $\mathbf{1}_{\nu_{0}}$ be $\mathcal{D}$-invariant for some $v_{0}$. Then $\mu_{v_{0}}$ is the Ashtekar-Lewandowski measure $\mu_{0}$.

Recall that regularity always means regularity w.r.t. $\mathcal{R}$, whereas $\mathcal{R}$ is taken from the nice enlarged structure data.

Corollary 7.2. Let $\pi$ be regular, and let there exist a (cyclic) $\mathcal{D}$-invariant vector in $\mathfrak{H}$.

Then there is a first-step decomposition of $\pi^{\prime}$, such that $\mu_{v_{0}}$ is the AshtekarLewandowski measure $\mu_{0}$ for some $v_{0}$ and $\mathbf{1}_{\nu_{0}}$ is (cyclic and) $\mathcal{D}$-invariant.

Proof. According to Lemma 2.3 and the agreements thereafter, we may find some $v_{0}$, such that $\mathbf{1}_{v_{0}}$ is $\mathcal{D}$-invariant (and cyclic). Now use the proposition above.

Note that, as mentioned earlier, we do not distinguish between the $\mathcal{D}$-invariances on equivalent representations. More precisely, we should say in the corollary above: There is an isomorphism $U: \mathfrak{H} \longrightarrow \mathfrak{H}^{\prime}$ with $\mathfrak{H}^{\prime}=\bigoplus_{\nu \in \mathrm{N}} L_{2}\left(\overline{\mathcal{A}}, \mu_{\nu}\right)$ for certain measures $\mu_{\nu}$ on $\overline{\mathcal{A}}$, such that $\left.U \circ \pi^{\prime}\right|_{C(X)} \circ U^{-1}$ is cyclic on each $L_{2}\left(\overline{\mathcal{A}}, \mu_{v}\right)$ with cyclic vector $\mathbf{1}_{v}$; moreover, $\mu_{v_{0}}$ equals $\mu_{0}$ for some $\nu_{0} \in \mathrm{N}$ and $U \pi^{\prime}\left(\alpha_{\varphi}\right) U^{-1} \mathbf{1}_{\nu_{0}}=\mathbf{1}_{\nu_{0}}$ for all $\varphi \in \mathcal{D}$.

Before we will be able to prove the proposition above, we have to provide two estimates.

Lemma 7.3. Let $T \in \mathcal{M}_{\gamma}$ be a gauge-variant spin network state, and let $\phi$ be some representation occurring in T. Denote the Casimir eigenvalue w.r.t. $\phi$ by $\lambda_{\phi}$ and set $n:=\operatorname{dim} \mathfrak{g}$. Finally, define $\eta: \mathbb{R}_{+} \longrightarrow \mathbb{R}_{+}$according to Lemma B.2.

Then there is a one-parameter group $w_{t}$ of Weyl operators, such that, for each $t_{0}>0$ and each even $J \in \mathbb{N}_{+}$, there are $(2 n)^{J}$ diffeomorphisms $\varphi_{\varrho}$, such that

$$
\left\|\frac{1}{(2 n)^{J}} \sum_{\varrho}\left(\alpha_{\varphi_{\varrho}}^{-1}\left(w_{t}\right)-\mathrm{e}^{-\frac{1}{2} \lambda_{\phi} J t^{2}} \mathbf{1}\right) T\right\|_{\infty} \leq\|T\|_{\infty}\left(\mathrm{e}^{\eta\left(t_{0}\right) J t^{4}}-1\right)
$$

for all $|t|<t_{0}$.

Proof. Fix some edge $\gamma \in \gamma$, such that $\phi$ is the representation carried by $\gamma$ in $T$. Let, according to Lemma B.2, $\left\{X_{i}\right\}_{i=1}^{n}$ be a basis of the Lie algebra $\mathfrak{g}$ of $\mathbf{G}$, such that $-\frac{1}{n} \sum_{i} \phi\left(X_{i}\right) \phi\left(X_{i}\right)$ is (up to the prefactor) the Casimir operator $\phi$. Define

$$
c_{\phi, \mathfrak{g}}(t):=\frac{1}{2 n} \sum_{i=1}^{n}\left(\phi\left(\mathrm{e}^{t X_{i}}\right)+\phi\left(\mathrm{e}^{-t X_{i}}\right)\right) \equiv \frac{1}{2 n} \sum_{i=1}^{2 n} \phi\left(\mathrm{e}^{t X_{i}}\right) \equiv \frac{1}{2 n} \sum_{i=1}^{2 n} \phi\left(\mathrm{e}^{-t X_{i}}\right)
$$

with $X_{i+n}:=-X_{i}$. According to Proposition 6.21, choose some interval $I \subseteq[0,1]$, for each $i=1, \ldots, 2 n$ some appropriate surface $S_{i}$ disjoint to im $\gamma$ and some $s \in \mathbb{Z}$. 
Moreover, let $\mathfrak{d}_{i}: M \longrightarrow \mathfrak{g}$ be the constant function of value $\frac{1}{2} X_{i}$, and fix some strictly increasing sequence $\left(\tau_{j}\right)_{j \in \mathbb{N}_{+}} \subseteq I$.

We are now going to consider the one-parameter group

$$
w_{t}:=\prod_{i=1}^{2 n} w_{i, t} \equiv \prod_{i=1}^{2 n} w_{E_{\mathfrak{o}_{i}}(t)}^{S_{i}, \sigma_{S_{i}}}
$$

In fact, this is a one-parameter group: All the $S_{i}$ are disjoint, whence $w_{i, t}$ and $w_{i^{\prime}, t^{\prime}}$ commute by Lemma 3.26. ${ }^{27}$

Fix now some positive even integer $J$ and some positive $t_{0}$. By the choice of $S_{i}$ and of $\left(\tau_{j}\right)$, for each $\varrho:[1, J] \longrightarrow[1,2 n]$ there is a diffeomorphism $\varphi_{\varrho} \in \mathcal{D}$ with the properties described in Proposition 6.21. In particular, since $\varphi_{\varrho}(\gamma)$ intersects $S:=\bigcup_{i} S_{i}$ completely transversally, the minimal $S$-admissible decomposition of $\varphi_{\varrho}(\gamma)$ contains $S$-external edges only. More explicitly, it equals $\varphi_{\varrho}\left(\gamma_{0}\right) \cdots \varphi_{\varrho}\left(\gamma_{J}\right)$ with $\gamma_{0}=\left.\gamma\right|_{\left[0, \tau_{1}\right]}$, $\gamma_{j}=\left.\gamma\right|_{\left[\tau_{j}, \tau_{j+1}\right]}$ and $\gamma_{J}=\left.\gamma\right|_{\left[\tau_{J}, 1\right]}$. By $\varphi_{\varrho}\left(\gamma\left(\tau_{j}\right)\right) \in S_{\varrho(j)}$ for all $j$, we see that $\varphi_{\varrho}\left(\gamma_{j}\right)$ starts in $S_{\varrho(j)}$ and ends in $S_{\varrho(j+1)}$ (with the obvious exceptions for $j=0$ and $j=J$ ). Since, moreover, by construction, $\sigma^{+}\left(S_{\varrho(j)}, \varphi_{\varrho}\left(\gamma_{j-1}\right)\right)=(-1)^{j+s}=\sigma^{-}\left(S_{\varrho(j)}, \varphi_{\varrho}\left(\gamma_{j}\right)\right)$ for $j=1, \ldots, J$, we get

$$
\begin{aligned}
w_{t}\left(\alpha_{\varphi_{\varrho}}\left(\phi \circ \pi_{\gamma}\right)\right) & =w_{t}\left(\phi \circ \pi_{\varphi_{\varrho} \gamma}\right)=\bigotimes_{j=0}^{J} w_{t}\left(\phi \circ \pi_{\varphi_{\varrho} \gamma_{j}}\right) \\
& =\left(\phi \circ \pi_{\varphi_{\varrho} \gamma_{0}}\right) \otimes \bigotimes_{j=1}^{J}\left(\phi\left(\mathrm{e}^{(-1)^{j+s} X_{\varrho(j)} t}\right) \cdot\left(\phi \circ \pi_{\varphi_{\varrho} \gamma_{j}}\right)\right),
\end{aligned}
$$

hence

$$
\left[\alpha_{\varphi_{\varrho}}^{-1}\left(w_{t}\right)\right]\left(\phi \circ \pi_{\gamma}\right)=\left(\phi \circ \pi_{\gamma_{0}}\right) \otimes \bigotimes_{j=1}^{J}\left(\phi\left(\mathrm{e}^{(-1)^{j+s} X_{\varrho(j)} t}\right) \cdot\left(\phi \circ \pi_{\gamma_{j}}\right)\right)
$$

and

$$
\begin{aligned}
\frac{1}{(2 n)^{J}} \sum_{\varrho}\left[\alpha_{\varphi_{\varrho}}^{-1}\left(w_{t}\right)\right]\left(\phi \circ \pi_{\gamma}\right) & =\left(\phi \circ \pi_{\gamma_{0}}\right) \otimes \bigotimes_{j=1}^{J} \frac{1}{2 n} \sum_{i=1}^{2 n} \phi\left(\mathrm{e}^{(-1)^{j+s} X_{i} t}\right) \cdot\left(\phi \circ \pi_{\gamma_{j}}\right) \\
& =\left(\phi \circ \pi_{\gamma_{0}}\right) \otimes \bigotimes_{j=1}^{J} c_{\phi, \mathfrak{g}}(t) \cdot\left(\phi \circ \pi_{\gamma_{j}}\right) .
\end{aligned}
$$

Here, we used

$$
\sum_{\varrho:[1, J] \longrightarrow[1,2 n]} \bigotimes_{j=1}^{J} a_{\varrho(j), j}=\bigotimes_{j=1}^{J} \sum_{i=1}^{2 n} a_{i, j}
$$

27 If we choose some $E(t): M \longrightarrow \mathbf{G}$ with $E(t)=E_{\mathfrak{d}_{i}}(t)$ on each $S_{i}$ and define $\sigma_{S}$ to be the joint intersection function of $S_{1}, \ldots, S_{2 n}$, we get $w_{t}=w_{E(t)}^{S, \sigma_{S}}$. Recall that we assumed that $\mathcal{R}$ contains not only the "genuine" subgroups in $\mathcal{W}$, but also the finite products of such subgroups, provided they mutually commute. Therefore, it is not important that $E(t)$ is possibly not included in $\Delta(S)$. 
By assumption, we have $T=T_{\gamma} \otimes T_{\gamma^{\prime}}=\sqrt{\operatorname{dim} \phi}\left(\phi \circ \pi_{\gamma}\right)_{l}^{k} \otimes T_{\gamma^{\prime}}$ for some matrix indices $k, l$ and some $T_{\boldsymbol{\gamma}^{\prime}} \in \mathcal{M}_{\boldsymbol{\gamma}^{\prime}}$ with $\boldsymbol{\gamma}^{\prime}=\boldsymbol{\gamma} \backslash\{\boldsymbol{\gamma}\}$. Additionally using $S \cap \operatorname{im}\left(\varphi_{\varrho} \boldsymbol{\gamma}^{\prime}\right)=\varnothing$ and $\left\|\phi_{l}^{k}\right\|_{\infty} \leq\|\phi\|_{\infty}$, we get

$$
\begin{aligned}
& \left\|\frac{1}{(2 n)^{J}} \sum_{\varrho}\left(\alpha_{\varphi_{\varrho}}^{-1}\left(w_{t}\right)-\mathrm{e}^{-\frac{1}{2} \lambda_{\phi} J t^{2}} \mathbf{1}\right) T\right\|_{\infty} \\
& \leq\left\|\sqrt{\operatorname{dim} \phi}\left(\frac{1}{(2 n)^{J}} \sum_{\varrho}\left(\alpha_{\varphi_{\varrho}}^{-1}\left(w_{t}\right)-\mathrm{e}^{-\frac{1}{2} \lambda_{\phi} J t^{2}} \mathbf{1}\right)\left(\phi \circ \pi_{\gamma}\right)\right)_{l}^{k} \otimes T_{\gamma^{\prime}}\right\|_{\infty} \\
& \leq\left\|T_{\gamma}\right\|_{\infty}\left(\mathrm{e}^{\eta\left(t_{0}\right) J t^{4}}-1\right)\left\|T_{\gamma^{\prime}}\right\|_{\infty}
\end{aligned}
$$

for all $|t|<t_{0}$, by Lemma B.2 and the surjectivity [14] of $\pi_{v}: \overline{\mathcal{A}} \longrightarrow \mathbf{G}^{\# v}$ for every hyph $v$.

Corollary 7.4. Let $\pi^{\prime}$ be some representation of $\mathfrak{A}_{\text {Diff }}$, let $T \in \mathcal{M}_{\gamma}$ be a nontrivial gauge-variant spin network and let $\psi \in \mathfrak{H}$ be $\mathcal{D}$-invariant with $\langle\psi, \pi(T) \psi\rangle_{\mathfrak{H}} \neq 0$.

Then there is a one-parameter group $w_{t}$ of Weyl operators and $\varepsilon, t_{0}>0$, such that for all $0 \neq|t|<t_{0}$ there is a diffeomorphism $\varphi$ with

$$
\left|\left\langle\psi, \pi\left(\left(w_{t}-\mathbf{1}\right)\left(\alpha_{\varphi} T\right)\right) \psi\right\rangle_{\mathfrak{H}}\right| \geq \varepsilon .
$$

Proof. - Set $\varepsilon:=\min \left\{\frac{1}{2}, \frac{1}{4}\left|\langle\psi, \pi(T) \psi\rangle_{\mathfrak{H}}\right|\right\}>0$. Fix $\tau_{0}>0$ and some non-trivial irreducible representation $\phi$ occurring in $T$. Next, choose positive real $\tau_{2}$ and $\tau_{4}$, such that (using the $\eta\left(\tau_{0}\right)$ as given in Lemma 7.3)

$$
\|\psi\|_{\mathfrak{H}}^{2}\|T\|_{\infty}\left(\mathrm{e}^{\eta\left(\tau_{0}\right) \tau}-1\right)<\varepsilon \quad \text { for all }|\tau|<\tau_{4}
$$

and

$$
\mathrm{e}^{-\frac{1}{2} \lambda_{\phi} \tau}<\varepsilon \quad \text { for all }|\tau|>\tau_{2}
$$

Define now

$$
J_{0}:=\frac{1}{2} \sqrt{2 \tau_{2} \tau_{4}} \quad \text { and } \quad t_{0}:=\min \left\{\frac{1}{\tau_{2}} J_{0}, \sqrt[3]{J_{0}}, \tau_{0}\right\} .
$$

We say that $(J, t) \in \mathbb{N}_{+} \times \mathbb{R}$ is an admissible pair iff

$$
0<|t|<t_{0} \quad \text { and } \quad \frac{J_{0}}{t^{3}} \leq J \leq 2 \frac{J_{0}}{t^{3}} .
$$

As one checks easily, the admissibility of $(J, t)$ implies $J t^{2}>\tau_{2}$ and $J t^{4}<\tau_{4}$. Moreover, for every $0<|t|<t_{0}$, there is an even $J(t) \in \mathbb{N}_{+}$such that $(t, J(t))$ is admissible.

- Choose now a one-parameter subgroup $w_{t}$ of Weyl operators and, for all positive integers $J$, some diffeomorphisms $\varphi_{\varrho}, \varrho \in \mathrm{P}_{J}$, as in Lemma 7.3. Consequently, we have

$$
\left|\frac{1}{(2 n)^{J}} \sum_{\varrho \in \mathrm{P}_{J}}\left\langle\psi, \pi\left(\left(\alpha_{\varphi_{\varrho}}^{-1}\left(w_{t}\right)-\mathrm{e}^{-\frac{1}{2} \lambda_{\phi} J t^{2}} \mathbf{1}\right) T\right) \psi\right\rangle_{\mathfrak{H}}\right|
$$




$$
\begin{aligned}
& \leq\left\|\pi\left(\frac{1}{(2 n)^{J}} \sum_{\varrho \in \mathrm{P}_{J}}\left(\alpha_{\varphi_{\varrho}}^{-1}\left(w_{t}\right)-\mathrm{e}^{-\frac{1}{2} \lambda_{\phi} J t^{2}} \mathbf{1}\right) T\right)\right\|_{\mathcal{B}(\mathfrak{H})}\|\psi\|_{\mathfrak{H}}^{2} \\
& \leq\left\|\frac{1}{(2 n)^{J}} \sum_{\varrho \in \mathrm{P}_{J}}\left(\alpha_{\varphi_{\varrho}}^{-1}\left(w_{t}\right)-\mathrm{e}^{-\frac{1}{2} \lambda_{\phi} J t^{2}} \mathbf{1}\right) T\right\|_{\infty}\|\psi\|_{\mathfrak{H}}^{2} \\
& \leq\left(\mathrm{e}^{\eta\left(\tau_{0}\right) J t^{4}}-1\right)\|T\|_{\infty}\|\psi\|_{\mathfrak{H}}^{2} \\
& <\varepsilon
\end{aligned}
$$

and, using $(1-\varepsilon)\left|\langle\psi, \pi(T) \psi\rangle_{\mathfrak{H}}\right| \geq \frac{1}{2}\left|\langle\psi, \pi(T) \psi\rangle_{\mathfrak{H}}\right| \geq 2 \varepsilon$,

$$
\begin{aligned}
& \left|\frac{1}{(2 n)^{J}} \sum_{\varrho \in \mathrm{P}_{J}}\left(1-\mathrm{e}^{-\frac{1}{2} \lambda_{\phi} J t^{2}}\right)\langle\psi, \pi(T) \psi\rangle_{\mathfrak{H}}\right| \\
& \quad=\left(1-\mathrm{e}^{-\frac{1}{2} \lambda_{\phi} J t^{2}}\right)\left|\langle\psi, \pi(T) \psi\rangle_{\mathfrak{H}}\right| \geq(1-\varepsilon)\left|\langle\psi, \pi(T) \psi\rangle_{\mathfrak{H}}\right| \geq 2 \varepsilon
\end{aligned}
$$

for all admissible pairs $(J, t)$.

- Altogether, we have for all admissible pairs $(J, t)$,

$$
\begin{aligned}
& \left|\frac{1}{(2 n)^{J}} \sum_{\varrho \in \mathrm{P}_{J}}\left\langle\psi, \pi\left(\left(\alpha_{\varphi_{\varrho}}^{-1}\left(w_{t}\right)-\mathbf{1}\right)(T)\right) \psi\right\rangle_{\mathfrak{H}}\right| \\
& \geq|| \frac{1}{(2 n)^{J}} \sum_{\varrho \in \mathrm{P}_{J}}\left(1-\mathrm{e}^{-\frac{1}{2} \lambda_{\phi} J t^{2}}\right)\langle\psi, \pi(T) \psi\rangle_{\mathfrak{H}} \mid \\
& \quad-\left|\frac{1}{(2 n)^{J}} \sum_{\varrho \in \mathrm{P}_{J}}\left\langle\psi, \pi\left(\left(\alpha_{\varphi_{\varrho}}^{-1}\left(w_{t}\right)-\mathrm{e}^{-\frac{1}{2} \lambda_{\phi} J t^{2}} \mathbf{1}\right)(T)\right) \psi\right\rangle_{\mathfrak{H}}\right| \mid \\
& >\varepsilon .
\end{aligned}
$$

- Finally, for all $0<|t|<t_{0}$, we may choose a $J(t)$ providing an admissible pair $(J(t), t)$. By the lines above, there is some $\varphi \in\left\{\varphi_{\varrho} \mid \varrho \in \mathrm{P}_{J(t)}\right\}$, such that

$$
\left|\left\langle\psi, \pi\left(\left(w_{t}-\mathbf{1}\right)\left(\alpha_{\varphi} T\right)\right) \psi\right\rangle_{\mathfrak{H}}\right|=\left|\left\langle\psi, \pi\left(\left(\alpha_{\varphi}^{-1}\left(w_{t}\right)-\mathbf{1}\right) T\right) \psi\right\rangle_{\mathfrak{H}}\right|>\varepsilon
$$

using the $\mathcal{D}$-invariance of $\psi$.

Proof of Proposition 7.1. Corollary 7.4 shows that $\mathcal{W}^{\prime}$ splits $\mathcal{W}$ at $\mathbf{1}_{v_{0}}$ for every nontrivial gauge-variant spin network state $T$ with $\langle\mathbf{1}, T\rangle_{\nu_{0}} \equiv\left\langle\mathbf{1}_{v_{0}}, \pi(T) \mathbf{1}_{\nu_{0}}\right\rangle_{\mathfrak{H}} \neq 0$. Hence, $\mathcal{W}^{\prime}$ splits $\mathcal{W}$ at $\mathbf{1}_{\nu_{0}}$, by Lemma 3.3. Since $\pi$ is regular, Proposition 2.21 gives the assertion.

7.2. Naturality. Now we are using nice enlarged structure data and assume additionally that

- $\mathcal{D}$ contains at least the stratified isomorphisms described in Subsect. 6.4 and at least those necessary to keep Proposition 6.14 valid;

- $\mathcal{S}$ contains at most all $\mathcal{D}$-orbits of semianalytic subsets in $M$ having a finite wide triangulation and being of lower dimension than $M$, but contains at least the $q$-balls and $q$-simplices with $q<\operatorname{dim} M$. 


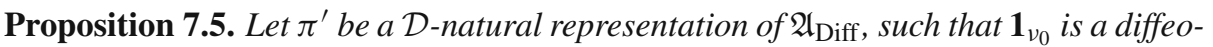
morphism-invariant vector and $\mu_{v_{0}}$ equals $\mu_{0}$ for some $\nu_{0} \in \mathrm{N}$.

Then the restriction of $\pi^{\prime}$ to $\mathfrak{H}_{\nu_{0}}$ is the fundamental representation $\pi_{0}$, i.e., we have $P_{v} \pi^{\prime}(a)=\pi_{0}(a) P_{\nu}$ for all $a \in \mathfrak{A}_{\text {Diff. }}$.

The proof of the proposition will use several steps we are now going to write down in separate lemmata. For this, throughout the whole section, we will assume that $\pi^{\prime}$ is a $\mathcal{D}$-natural representation of $\mathfrak{A}_{\text {Diff }}$ having some $\mathbf{1}_{\nu_{0}}$ as a $\mathcal{D}$-invariant vector. Moreover, $\mu_{\nu_{0}}$ equal $\mu_{0}$. Finally, as usual, we set $\pi:=\left.\pi^{\prime}\right|_{\mathfrak{A}}$.

Lemma 7.6. Let $S_{1}$ and $S_{2}$ be elements in $\mathcal{S}$ having orientations $\sigma_{S_{1}}$ and $\sigma_{S_{2}}$. Assume that they are oriented-strata equivalent. Finally, let $g \in \mathbf{G}$ be some element and $d_{i}: S_{i} \longrightarrow \mathbf{G}$ for $i=1,2$ be the constant function with value $g$.

Then $w_{d_{1}}^{S_{1}, \sigma_{S_{1}}}$ is a $\pi_{v_{0}}$-unit $\left(\pi_{v_{0}}\right.$-scalar) iff $w_{d_{2}}^{S_{2}, \sigma_{S_{2}}}$ is a $\pi_{v_{0}}$-unit $\left(\pi_{v_{0}}\right.$-scalar $)$.

Proof. Let $\varphi$ be a product of localized stratified isomorphisms mapping $S_{1}$ onto $S_{2}$ as well as their orientations. Then

$$
\alpha_{\varphi}\left(w_{d_{1}}^{S_{1}, \sigma_{S_{1}}}\right)=w_{\varphi\left(d_{1}\right)}^{\varphi\left(S_{1}\right), \varphi\left(\sigma_{S_{1}}\right)}=w_{d_{2}}^{S_{2}, \sigma_{S_{2}}} .
$$

Now, the assertion follows from Corollary 2.10.

Lemma 7.7. Let $S$ some subset of $M$, such that $S$ and $\partial S$ are connected embedded submanifolds in $M$ (without boundary) and that $\bar{S}$ is an embedded submanifold in $M$ having boundary $\partial S$. Moreover, assume that $S$ has one of its natural orientations. Finally, let $w=w_{d}^{S, \sigma_{S}}$ for some constant $d \in \Delta(S)$.

Then $P_{\nu_{0}} \pi(w) \mathbf{1}_{\nu_{0}} \in \mathfrak{H}_{\nu_{0}} \equiv L_{2}\left(\overline{\mathcal{A}}, \mu_{0}\right)$ is orthogonal to all non-trivial gauge-variant spin network states that are not based on an edge $\gamma$ whose image equals $S$, a $S$ or $\bar{S}$.

Recall that no edge of a gauge-variant spin network is labelled with the trivial representation.

Proof. Let $T$ be a gauge-variant spin network state in $\mathcal{M}_{\boldsymbol{\gamma}}$. There are two main cases:

- $\operatorname{im} \gamma$ neither equals $S, \partial S$ nor $\bar{S}$.

By Proposition 6.22, there is an infinite number of localized stratified diffeomorphisms $\varphi_{i}$, leaving $S$ (including its orientation and $d$ ) and each edge of $\gamma$ except for some $\gamma$ invariant, and forming a hyph $\left\{\varphi_{i}(\gamma)\right\}_{i}$. Consequently, $\alpha_{\varphi_{i}} T$ and $\alpha_{\varphi_{j}} T$ are orthogonal for $i \neq j$. Moreover, each $\alpha_{\varphi_{i}}$ commutes with $w$. Therefore, by Lemma 2.17, $P_{\nu_{0}} \pi(w) \mathbf{1}_{v_{0}}$ is orthogonal to $T$.

- $\operatorname{im} \gamma$ equals $S, \partial S$ or $\bar{S}$.

Assume first im $\boldsymbol{\gamma}=\partial S$. Since im $\boldsymbol{\gamma}$ is compact, $\partial S$ has to be compact as well. Hence, it is isomorphic to $S^{1}$. After a possibly necessary re-orientation of $\boldsymbol{\gamma}$, the product of all paths in $\gamma$ is a closed edge $\gamma$ with image $\partial S$. Assume that $T$ is not $(\gamma, \phi)$-based for some $\phi$. Then there is some vertex $m$ in $\boldsymbol{\gamma}$, where the adjacent edges at $m$ are either labelled with different representations or carry non-matching indices. Now, as in the previous case, but this time by Proposition 6.23, there are infinitely many localized stratified diffeomorphisms $\varphi_{i}$, leaving the sets $S$ (including orientation and $d$ ) and $\partial S$ invariant; they simply move $m$ along $\partial S$ stretching $\partial S$ a bit. By Lemma 3.4, any two $\alpha_{\varphi_{i}} T$ and $\alpha_{\varphi_{j}} T$ with $i \neq j$ are orthogonal. Since each $\alpha_{\varphi_{i}}$ commutes with $w$, Lemma 2.17 proves the orthogonality of $P_{v_{0}} \pi(w) \mathbf{1}_{\nu_{0}}$ and $T$.

The case of im $\gamma=S$ is completely analogous. For im $\gamma=\bar{S}$ we may additionally get the case of an embedded interval. However, this is analogous as well. 
Immediately from the proof of the lemma above and that of Proposition 6.22, we get

Corollary 7.8. Let $S$ be some subset of $M$ and $w_{d}^{S, \sigma_{S}} \in \mathcal{W}$ be any Weyl operator. Moreover, let $\boldsymbol{\gamma}$ be a graph not contained in the closure of $S$.

Then $P_{\nu_{0}} \pi(w) \mathbf{1}_{\nu_{0}} \in \mathfrak{H}_{\nu_{0}} \equiv L_{2}\left(\overline{\mathcal{A}}, \mu_{0}\right)$ is orthogonal to all non-trivial gauge-variant spin network states in $\mathcal{M}_{\boldsymbol{\gamma}}$.

We are now going to prove that the Weyl operators to open balls given some constant "labelling" $d$, are $\pi_{\nu_{0}}$-units. We start with the dimensions 0 and $3+$, but smaller than $\operatorname{dim} M$, proceed with dimension 1 and end up with dimension 2.

Corollary 7.9. Let $s<\operatorname{dim} M$ be some non-negative integer with $s \neq 1,2$, and let $S$ be an open or closed $s$-dimensional ball in $M$ given a nice orientation.

Then $w:=w_{d}^{S, \sigma_{S}}$ is a $\pi_{v_{0}}$-unit for every constant $d \in \Delta(S)$.

Proof. Let $\boldsymbol{\gamma}$ be a non-trivial graph. Since $s \neq 1,2$, neither $S, \partial S$ nor $\bar{S}$ equals im $\boldsymbol{\gamma}$. Thus, $P_{\nu_{0}} \pi(w) \mathbf{1}_{\nu_{0}}$ is orthogonal to each non-trivial $T \in \mathcal{M}_{\mathrm{SN}}$. Since $\mathcal{M}_{\mathrm{SN}}$ is a continuous $\mu_{0}$-generating system, $w$ is a $\pi_{\nu_{0}}$-scalar (see also Lemma 2.14).

To prove that $w$ is even a $\pi_{v_{0}}$-unit observe first that, by Propositions 6.10 and 6.14 , there is a stratified isomorphism $\varphi$ mapping $S$ onto itself, but reverting its orientation. Thus, $\alpha_{\varphi}(w)=w^{*}$, whence $w^{2}$ is a $\pi_{\nu_{0}}$-unit by Corollary 2.11. Since $\mathbf{G}$ is compact, there is a square root for any element. Re-doing the proof for $d_{1} \in \Delta(S)$ with $d_{1} d_{1}=d$ gives the assertion.

Lemma 7.10. Let $w \in \mathcal{W}$ be a Weyl operator for some quasi-surface $S$ and some constant $d \in \Delta(S)$, and let $\gamma$ be an analytic edge, such that $P_{\nu_{0}} \pi(w) \mathbf{1}_{v_{0}}$ is contained in the closure of $\operatorname{span} \mathcal{B}_{\gamma}$.

If the image of $\gamma$ is not completely contained in $S$, then $w$ is a $\pi_{v_{0}}$-scalar.

Proof. Let $m \in \operatorname{im} \gamma \backslash S$. If $\gamma$ is closed, we may assume that $m$ is not the base point of $\gamma$. Consider now for each $g \in \mathbf{G}$ the Weyl operator $w_{g, m}$ given by the quasi-surface $S_{m}:=\{m\}$, whereas the orientation of $S_{m}$ is chosen, such that the direction of $\gamma$ coincides with the orientation of $S_{m}$. Since $S_{m}$ and $S$ are disjoint, $w_{g, m}$ and $w$ commute. Moreover, by Corollary $7.9, w_{g, m}$ is a $\pi_{v_{0}}$-unit. Consequently, by Corollary $2.13, w_{g, m}$ leaves $P_{v_{0}} \pi(w) \mathbf{1}_{v_{0}}$ invariant.

- Let $m$ be not an endpoint of $\gamma$.

First of all, let $T=\left(T_{\gamma, \phi}\right)_{j}^{i} \in \mathcal{B}_{\gamma, \phi}$ for some non-trivial $\phi$ with $\phi_{k}=\phi$ for all $k$ and with $m$ being a vertex of $\boldsymbol{\gamma}$. One easily checks that

$$
w_{g, m}(T)=\sum_{r_{1}, r_{2}} \phi\left(g^{2}\right)_{r_{2}}^{r_{1}}\left(T_{\boldsymbol{\gamma}, \boldsymbol{\phi}}\right)_{\boldsymbol{j}\left(r_{1}\right)}^{\boldsymbol{i}\left(r_{2}\right)},
$$

whereas $\boldsymbol{i}(r)$ is the tuple of all $i_{k}$ where the index belonging to the edge leaving at $m$ is replaced by $r$. Hence,

$$
\begin{aligned}
\left\langle P_{\nu_{0}} \pi(w) \mathbf{1}_{\nu_{0}}, T\right\rangle_{\mu_{0}} & =\left\langle w_{g, m}^{*}\left(P_{v_{0}} \pi(w) \mathbf{1}_{\nu_{0}}\right), T\right\rangle_{\mu_{0}} \\
& =\left\langle P_{\nu_{0}} \pi(w) \mathbf{1}_{v_{0}}, w_{g, m}(T)\right\rangle_{\mu_{0}} \\
& =\sum_{r_{1}, r_{2}} \phi\left(g^{2}\right)_{r_{2}}^{r_{1}}\left\langle P_{\nu_{0}} \pi(w) \mathbf{1}_{v_{0}},\left(T_{\boldsymbol{\gamma}, \boldsymbol{\phi}}\right)_{\boldsymbol{j}\left(r_{1}\right)}^{\boldsymbol{i}\left(r_{2}\right)}\right\rangle_{\mu_{0}}
\end{aligned}
$$


for all $g \in \mathbf{G}$ and therefore, since square roots exist in $\mathbf{G}$,

$$
\begin{aligned}
\left\langle P_{\nu_{0}} \pi(w) \mathbf{1}_{\nu_{0}}, T\right\rangle_{\mu_{0}} & =\int_{\mathbf{G}}\left\langle P_{\nu_{0}} \pi(w) \mathbf{1}_{\nu_{0}}, T\right\rangle_{\mu_{0}} \mathrm{~d} \mu_{\text {Haar }}(g) \\
& =\sum_{r_{1}, r_{2}}\left\langle P_{\nu_{0}} \pi(w) \mathbf{1}_{\nu_{0}},\left(T_{\boldsymbol{\gamma}, \boldsymbol{\phi}}\right)_{\boldsymbol{j}\left(r_{1}\right)}^{\boldsymbol{i}\left(r_{2}\right)}\right\rangle_{\mu_{0}} \int_{\mathbf{G}} \phi(g)_{r_{2}}^{r_{1}} \mathrm{~d} \mu_{\text {Haar }}(g) \\
& =0 .
\end{aligned}
$$

Now, if $T=\left(T_{\boldsymbol{\gamma}, \boldsymbol{\phi}}\right)_{j}^{i} \in \mathcal{B}_{\gamma, \phi}$ for some non-trivial $\phi$ without $m$ being a vertex of $\boldsymbol{\gamma}$, we may refine $\boldsymbol{\gamma}$ by inserting $m$ as a new vertex. Then $T$ is a (finite) sum of $(\gamma, \phi)$ based gSNs each having $m$ as a vertex of the underlying graph. Using the just shown result, we get

$$
\left\langle P_{\nu_{0}} \pi(w) \mathbf{1}_{\nu_{0}}, T\right\rangle_{\mu_{0}}=0
$$

for all $(\gamma, \phi)$-based gauge-variant spin network states.

Altogether, this shows that $P_{v_{0}} \pi(w) \mathbf{1}_{\nu_{0}}$ is orthogonal to all non-trivial gaugevariant spin network states, i.e., $w$ is a $\pi_{v_{0}}$-scalar.

- Let $m$ be an endpoint of $\gamma$.

We argue analogously, using

$$
w_{g, m}(T)=\sum_{r} \phi(g)_{r}^{i_{1}}\left(T_{\boldsymbol{\gamma}, \boldsymbol{\phi}}\right)_{j}^{i(r)}
$$

if $m=\gamma(0)$, and similarly for $m=\gamma(1)$.

Corollary 7.11. Let $S$ be an open 1-dimensional ball in $M$ given a nice orientation. Then $w:=w_{d}^{S, \sigma_{S}}$ is a $\pi_{v_{0}}$-unit for every constant $d \in \Delta(S)$.

Proof. Let $\gamma$ be the edge whose interior is $S$ and choose one of its orientations. By Lemma 7.7, $P_{\nu_{0}} \pi(w) \mathbf{1}_{\nu_{0}}$ is orthogonal to all non-trivial gauge-variant spin network states that are not based on the edge $\gamma$. By Corollary 3.6, $P_{\nu_{0}} \pi(w) \mathbf{1}_{\nu_{0}}$ is contained in the closure of the span of $\gamma$-based gSNs. Since, however, the endpoints of $\gamma$ are not contained in $S$, Lemma 7.10 implies that $w$ is a $\pi_{v_{0}}$-scalar. Now, by Proposition 6.10, there is some $\varphi \in \mathcal{D}$ being the identity on $S$, but inverting the orientation of $S$, i.e., $\alpha_{\varphi}(w)=w^{*}$. Corollary 2.11 implies that $w^{2}$ is a $\pi_{v_{0}}$-unit. As above, the assertion follows since square roots exist in $\mathbf{G}$.

Corollary 7.12. Let $S$ be an open 2-dimensional ball in $M$ given a nice orientation.

Then $w:=w_{d}^{S, \sigma_{S}}$ is a $\pi_{v_{0}}$-unit for every constant $d \in \Delta(S)$.

Proof. The image of an edge $\gamma$ equals $S, \partial S$ or $\bar{S}$ iff $\gamma$ is a closed loop along $\partial S \cong S^{1}$. By Lemma 7.7, $P_{\nu_{0}} \pi(w) \mathbf{1}_{\nu_{0}}$ is orthogonal to all non-trivial gauge-variant spin network states not based on such a $\gamma$. Hence, we have $P_{\nu_{0}} \pi(w) \mathbf{1}_{v_{0}} \in \overline{\operatorname{span} \mathcal{B}_{\gamma}}$ by Corollary 3.6. Observe that im $\gamma \cap S=\varnothing$. Now argue as in Corollary 7.11.

Proposition 7.13. Let $S$ be a finitely widely triangulizable subset in $M$ having a natural orientation with $\operatorname{dim} S<\operatorname{dim} M$.

Then $w:=w_{d}^{S, \sigma_{S}}$ is a $\pi_{\nu_{0}}$-unit for every $d \in \Delta(S)$ being constant on $S$. 
Proof. - $S$ is an open $q$-simplex having a nice orientation.

By Corollary 6.11 and Proposition 6.14, $S$ is oriented-strata equivalent to a nicely oriented $q$-ball. So we get the assertion, since $q$-balls lead to Weyl operators that are $\pi_{v_{0}}$-units (Corollaries 7.9, 7.11 and 7.12) and since this property is inherited to all oriented-strata equivalent objects according to Lemma 7.6.

- $S$ is finitely widely triangulizable.

This means, by definition, $S$ is the finite disjoint union of nicely oriented simplices. Since disjoint unions lead to products of (commuting) Weyl operators (see Lemma 3.26), we get the assertion as well in the general case.

Proof of Proposition 7.5. Use Proposition 7.13 and Lemma 2.12, observing that each $w^{\prime} \in \mathcal{W}^{\prime}$ is a $\pi_{\nu_{0}}^{\prime}$-unit and that $\mathfrak{A}_{\text {Diff }}$ is generated by $\mathcal{W}, \mathcal{W}^{\prime}$ and $C(X)$.

\subsection{Classification.}

Definition 7.1. Enlarged structure data are called optimal iff they are nice and

- $M$ is at least three-dimensional;

- $\Delta(S)$ contains

- at most the constant functions together with their $\mathcal{E}$-orbits;

- D contains

- at least the stratified isomorphisms described in Subsect. 6.4,

- at least those necessary to keep Proposition 6.14 valid;

- $\mathcal{S}$ contains

- at least those hypersurfaces that are necessary to keep Proposition 6.21 valid,

- at least the $q$-balls and $q$-simplices for $q<\operatorname{dim} M$,

- at most all D-orbits of semianalytic subsets in $M$ having a finite wide triangulation and being of lower dimension than $M$.

Theorem 7.14. Let $\pi^{\prime \prime}$ be a representation of $\mathfrak{A}_{\text {Auto }}$ on $\mathfrak{H}$, such that $\pi:=\pi^{\prime \prime} \mid \mathfrak{A}_{\text {is regular }}$ and $\pi^{\prime}:=\left.\pi^{\prime \prime}\right|_{\mathfrak{A}_{\text {Diff }}}$ is $\mathcal{D}$-natural. Moreover, let there exist some $\mathcal{D}$-E -invariant vector in $\mathfrak{H}$ being cyclic for $\pi$. Finally, let optimal enlarged structure data be given.

Then $\pi^{\prime \prime}$ is unitarily equivalent to the fundamental representation of $\mathfrak{A}_{\text {Auto. }}$.

Proof. By Corollary 7.2, regularity and diffeomorphism invariance imply that there is a first-step decomposition of $\pi^{\prime}$, such that some $\mu_{\nu_{0}}$ is the Ashtekar-Lewandowski measure $\mu_{0}$ and that $\mathbf{1}_{\nu_{0}}$ is $\mathcal{D}$-invariant. Naturality, diffeomorphism invariance and Proposition 7.5 imply that each $w \in \mathcal{W}$ w.r.t. a constant $d \in \Delta(S)$ is a $\pi_{v_{0}}$-unit. As $\mathbf{1}_{\nu_{0}}$ is also $\mathcal{E}$-invariant, $w \in \mathcal{W}$ is a $\pi_{v_{0}}$-unit for every $d \in \Delta(S)$ by Lemma 2.4 and Proposition 3.38. Cyclicity gives the proof by Corollary 2.7 .

We remark that the results above can be directly extended to semianalytic sets having the same dimension as $M$. Of course, the triangulizability has to be guaranteed and the intersection functions have to be adjusted. The latter one can be done, e.g., by setting $\sigma_{S}(\gamma)$ for closed $S$ to be one iff $\gamma$ starts at the boundary of $S$ and then leaves $S$ nontangentially. The proofs, however, have to be modified accordingly. In particular, there is no longer an extra dimension available to mirror simplices and balls. Instead, we now use that there are diffeomorphisms mapping simplices (enlarged by one of its faces) onto two other, disjoint simplices whose union is the original simplex again (Proposition 6.13). The proofs that the corresponding Weyl operators are $\pi_{v}$-units, should now use the first statement of Corollary 2.11 and proceed inductively on the dimension. 
7.4. Discussion. Having now obtained the desired uniqueness theorem, we might ask whether the assumptions for it are reasonable.

\subsubsection{Structure data. First of all, let us consider the enlarged structure data.}

Lemma 7.15. The following enlarged structure data are optimal:

- $M$ is an at least three-dimensional analytic manifold;

- G is a nontrivial connected compact Lie group;

- $\mathcal{P}$ consists of all piecewise analytic paths in $M$;

- $\mathcal{E}$ contains the generalized gauge transforms; ${ }^{28}$

- $\mathcal{D}$ contains the stratified analytic diffeomorphisms in $M$;

- $\mathcal{S}$ contains the semianalytic sets in $M$ (together with their $\mathcal{D}$-orbits) having lower dimension than $M$ and having a finite wide triangulation;

- $\Sigma(S)$ contains the natural ${ }^{29}$ intersection functions of $S$;

- $\Delta(S)$ contains the constant functions on $M$ (together with their $\mathcal{E}$-orbits);

- $\mathcal{R}$ contains the one-parameter subgroups of Weyl operators consistent with $\mathcal{S},\{\Sigma(S)\}$, $\{\Delta(S)\}$.

From our point of view (see also the discussion in Subsect. 4.1), all ingredients are natural up to the restrictions on $\mathcal{S}$ and, maybe, on $M$ and $\Delta(S)$. The inclusion of semianalytic sets is reasonable, since the stratified diffeomorphisms map analytic hypersurfaces to semianalytic sets anyway. At the same time, the inclusion of lower-dimensional surfaces becomes natural. But, it would be desirable to at least replace the condition of wide triangulizability by the "standard" triangulizability, since in this case it is known that any semianalytic set is triangulizable. The requirement that each simplex in the triangulation is nicely oriented, is not too restrictive, since every naturally oriented, embedded surface is at least locally nicely oriented. The finiteness, on the other hand, cannot simply be dropped. This may at most be possible for compact $M$. In fact, then every semianalytic set has a compact closure and compact boundary. Then we may triangulize them finitely, by local finiteness. Redoing the procedure with the (lower-dimensional) semianalytic set given by the intersection of the original one with its boundary, we may successively get a finite decomposition of the original set into simplices. For non-compact $M$, this is no longer true. Simply take a hyperplane in $\mathbb{R}^{3}$ being triangulizable, of course, but not finitely. Well, although our proofs above have aimed at the finite case, we may extend the uniqueness result immediately to this example. Simply use that a hyperplane can be rotated onto itself inverting its orientation, and argue as in Corollary 7.9. In other words, it may be, as already mentioned above, that every analytic manifold is widely triangulizable; but even if not, there seems to be still some leeway in our argumentation above to keep the uniqueness given in the more general context. However, to explore this, several technical investigations in the field of semianalytic sets are necessary that go much beyond the scope of this paper.

We mentioned also the restriction that $M$ has to be at least three-dimensional. Well, for quantum gravity this is no problem at all, since the space-like hypersurfaces are three-dimensional. The space-time is even four-dimensional, although this does not

\footnotetext{
28 The statement remains true if we assume $\mathcal{E}$ to be any subset of $\overline{\mathcal{G}}$, e.g., just the (stratified analytic) gauge transforms of a particular principal fibre bundle.

29 To be precise, $\Sigma(S)$ should contain the natural intersection functions of $S$, if $S$ is a submanifold. In the general case, include all intersection functions that are joint intersection functions given by the nice intersection functions for the submanifolds forming the respective triangulation. Finally, if necessary, collect all intersection functions generated by the action of $\mathcal{D}$ on stratified sets of the types mentioned previously.
} 
seem relevant here, since we work with compact structure groups excluding the full covariant formulation of general relativity in four dimensions using the structure group $S O(3,1)$ or $S l(2, \mathbb{C})$. Nevertheless, we expect our result to be true in dimension 2 as well. In dimension 1 , one should check it by hand $-M$ can only be a line or a circle.

Another issue concerns the choice of functions $d \in \Delta(S)$ to label the stratified sets. Constant functions mark some minimal condition. On the other hand, our proofs in Subsect. 7.2 only go through for constant labellings. In fact, only these guarantee that diffeomorphisms mapping some $S$ onto itself preserve even its labelling. The most obvious way out might be to add some stronger notion of regularity. In particular, we might reuse the idea of step functions for the definition of integrals. This means we should approximate an arbitrary (sufficiently "smooth") function by simple functions, i.e., by sums of step functions, having sufficiently nice, disjoint supports. These sums now correspond to products of Weyl operators with constant labellings. Since these are represented identically, we would get the desired uniqueness for representations that are in this sense regular and if each $d$ can be approximated this way. However, this approximation again may be in conflict with the triangulation problem above. Therefore, at this point, we state only the directly given

Lemma 7.16. Besides nice enlarged structure data, assume that each $\Delta(S)$ consists of some subset of continuous functions $d: M \longrightarrow \mathbf{G}$. Equip $\Delta(S)$ with the supremum norm on $S$ induced by some fixed norm on $\mathbf{G}$. Assume there is some sequence $\left(d_{i}\right)_{i \in \mathbb{N}}$ with $d_{i} \rightarrow d$ in $\Delta(S)$, such that for all $i$ there are finitely many $S_{i, k_{i}}$ forming a decomposition of $S$ and each having a finite wide triangulation, whereas $d_{i}$ is constant on each $S_{i, k_{i}}$.

Then, given the assumptions of Theorem $7.14, \pi^{\prime}$ is equivalent to $\pi_{0}$, provided $\pi^{\prime}$ is $\Lambda^{0, S, \sigma_{S}}$-regular for all $S \in \mathcal{S}$ and $\sigma_{S} \in \Sigma(S)$.

Recall that $\pi_{0}$ itself is always $\Lambda^{0, S, \sigma_{S}}$-regular, i.e., if $d_{i}$ converges pointwise on $S$ to $d$, then the corresponding Weyl operators converge weakly.

Proof. Let $d, S$ and $\sigma_{S}$ be fixed. The Weyl operators corresponding to $S_{i, k_{i}}$ and $\left.d_{i}\right|_{S_{i, k_{i}}}$ are even $\pi_{v_{0}}$-units according to the proof of Theorem 7.14, hence each $w_{d_{i}}^{S, \sigma_{S}}$ as well, by Lemma 3.26. Proposition 2.22 and the $\Lambda^{0, S, \sigma_{S}}$-regularity imply that $w_{d}^{S, \sigma_{S}}$ is a $\pi_{\nu_{0}}$-unit as well. Corollary 2.7 gives the proof.

7.4.2. Further assumptions. Let us now say a few words about the other assumptions of Theorem 7.14. That we restrict ourselves to cyclic representations, is no restriction at all, since any (non-degenerate) representation can be decomposed into cyclic ones. Rather, the assumption that there is a cyclic vector being at the same time diffeomorphism invariant, is a restriction. This means that we only consider theories having a diffeomorphism invariant "vacuum". Well, this may be justified by the corresponding invariance of general relativity leading to some special kind of quantum geometry. Next, we assumed at least the "standard" regularity mapping weakly continuous one-parameter subgroups into weakly continuous ones. It may be desirable to drop this assumption; however, even in the classical theory of quantum mechanics, the Stone-von Neumann theorem relies on the regularity assumption. Indeed, it is very difficult to prove results without referring to it. However, in our case, there may be some hope, since the diffeomorphism group is that large and may thence identify so many objects in order to, possibly, replace some or all of the regularity assumptions. The naturality of the action of diffeomorphisms is discussed below. 
7.4.3. Improvements. Finally, we would like to emphasize that we were able to drop a crucial assumption and to weaken another made in the paper [35] by Sahlmann and Thiemann:

First of all, we did not need any assumptions about the domains of the operators. This was possible, since we are working with the exponentiated Weyl operators from the very beginning. The only point, where we went down to the non-exponentiated regime, was in Subsect. 7.1 (and Appendix B). But even there, we did not do this for generators of the represented Weyl operators. In fact, we did only use results for the convergence of the genuine Weyl operators w.r.t. the supremum norm. This way, we get some "analytic" convergence at the exponentiated level that, afterwards, leads to the emergence of the Ashtekar-Lewandowski measure by splitting and regularity.

Secondly, we significantly weakened the assumptions on the representation of the diffeomorphisms. Although we re-used the name "natural" representation, our definition imposes much less restrictions than that in [35]. There, the action of diffeomorphisms is said to be natural if it is the pull-back representation of $\mathcal{D}$ on each $L_{2}\left(\overline{\mathcal{A}}, \mu_{v}\right)$. This, however, is well-defined only if the pull-back action of $\mathcal{D}$ is well-defined on $L_{2}\left(\overline{\mathcal{A}}, \mu_{\nu}\right)$. In fact, in general, it is not. To see this, we use again the general notation of Sect. 2. Namely, let $\mu_{v}$ be the Dirac measure at some $x \in X$ being not invariant w.r.t. $\mathcal{W}^{\prime}$. Then $\mathfrak{H}_{v}=L_{2}\left(X, \mu_{v}\right)$ is isomorphic to $\mathbb{C}$ by $\psi \longmapsto \psi(x)$ for any measurable function $\psi$ on $X$. Take some $w^{\prime} \in \mathcal{W}^{\prime}$ with $\xi_{w^{\prime}}(x) \neq x$, and let the even continuous function $\psi$ be one at $\xi_{w^{\prime}}(x)$, but zero at $x$. Therefore, $\psi=0$ in $\mathfrak{H}_{\nu}$, but, at the same time, $\left(w^{\prime}(\psi)\right)(x)=\psi\left(\xi_{w^{\prime}}(x)\right)=1$, hence $w^{\prime}(\psi)=1$ in $\mathfrak{H}_{v}$. In other words, the extension of the pull-back mapping from $C(X)$ to $L_{2}\left(X, \mu_{v}\right)$ is ill defined. Additionally, one sees immediately, that, even if the pull-back representation is well defined, it is unitary only if $\mu_{v}$ is $\mathcal{W}^{\prime}$-invariant. This, of course, restricts the possible measures drastically. We, instead, defined naturality (see Definition 2.5) much less restrictive. Firstly, we do not refer to the pull-back representation at all. Secondly, before we impose conditions on the projection of $\pi^{\prime}$ to certain $\mathfrak{H}_{\nu}$, we check whether $\mathfrak{H}_{v}$ is invariant w.r.t. $\mathcal{W}^{\prime}$. Only then and only if $\mu_{\nu_{1}}$ and $\mu_{\nu_{2}}$ coincide, we, thirdly, require that the respective projections of $\pi^{\prime}$ coincide. This way, the problems indicated above are circumvented.

Nevertheless, one should think why one required naturality at all in our case $X=\overline{\mathcal{A}}$ and $\mathcal{W}^{\prime} \cong \mathcal{D}$. Recall that there are three different objects to be considered: the continuous functions on $\overline{\mathcal{A}}$, the Weyl operators $w \in \mathcal{W}$ and the diffeomorphisms $\alpha_{\varphi} \in \mathcal{W}^{\prime}$. The first two of them are dynamical, the last one is just a constraint. Therefore, it is reasonable to distinguish between them. For instance, it is not required that $\pi^{\prime}$ is regular. In fact, the diffeomorphisms act arbitrarily non-continuously on $\mathfrak{H}$ already in the fundamental representation: Given some $\psi \in L_{2}\left(\overline{\mathcal{A}}, \mu_{0}\right)$, say a spin network state on some graph $\boldsymbol{\gamma}$, then $\alpha_{\varphi}(\psi)$ is orthogonal to $\psi$ provided $\boldsymbol{\gamma}$ is not preserved by $\varphi$ (actually being a negligible restriction). Moreover, since $C(\overline{\mathcal{A}})$ is continuous, in any case, we may decompose the restriction of any representation to $C(\overline{\mathcal{A}})$ into canonical representations on $\overline{\mathcal{A}}$ w.r.t. certain measures. It now may be conceivable that, if the continuous functions on $\overline{\mathcal{A}}$ cannot distinguish between two of these addends, then the purely kinematical, constraining part cannot either. In other words, if two measures in the first-step decomposition coincide, then the induced representations should be identified. There is no obvious reason why diffeomorphisms should not keep the addends of the first-step decomposition invariant but, there is also no reason why they should. Therefore, although it might be reasonable to restrict oneself to natural representations of diffeomorphisms, this assumption does not seem to be absolutely desirable. If we do this, however, observe that the arguments above should not be applied to the Weyl operators. Indeed, first of all, these are dynamical 
objects, and, secondly, they act on a higher level, namely, on $\overline{\mathcal{A}}$ affected by the dynamics and not on the paths being the ultimate constituents of the theory and being the domain for the homomorphisms in $\overline{\mathcal{A}}$.

7.4.4. Main open issues. Of course, the remarks above are not at all final answers why to consider just these assumptions. At least from the mathematical point of view, it would be highly desirable to have more general results available. We have given some hints here for direct extensions, however, the field is still open, in particular:

Question 1. Is naturality w.r.t. diffeomorphisms necessary?

Question 2. Is regularity w.r.t. Weyl operators necessary?

Acknowledgements. The author thanks Abhay Ashtekar, Jerzy Lewandowski, Stefan Müller, Andrzej Okołów, Hans-Bert Rademacher, Hanno Sahlmann, Konrad Schmüdgen, Matthias Schwarz, Thomas Thiemann, Rainer Verch and Elmar Wagner for fruitful discussions. Moreover, the author is very grateful to Garth Warner for his remarks and, in particular, for pointing out a mistake in an earlier version of this article. Fortunately, all the results kept valid. Additionally, the author thanks the three anonymous referees for their very valuable comments and suggestions helping him to improve the article. The author has been supported in part by the Emmy-Noether-Programm (grant FL 622/1-1) of the Deutsche Forschungsgemeinschaft.

\section{Appendix}

\section{A. Continuity Criterion}

Lemma A.1. Let $Y$ be some sequential topological space. Let $X$ be a Banach space and let $\lambda: Y \longrightarrow \mathcal{B}(X)$ be some map. Moreover, let $\|\lambda(\cdot)\|: Y \longrightarrow \mathbb{R}$ be locally bounded. Assume, finally, that there is some subset $E \subseteq X$, such that $y \longmapsto \lambda(y) e$ is continuous for all $e \in E$ and that span $E$ is dense in $X$.

Then $y \longmapsto \lambda(y) x$ is continuous for all $x \in X$.

Proof. Fix some $y^{\prime} \in Y$ and choose a neighbourhood $U$ of $y^{\prime}$, such that $\|\lambda(\cdot)\|$ is bounded on $U$, say, by $c$. Let now $\varepsilon>0$ and $x \in X$. Then there are $x_{1}, x_{2} \in X$ with $x_{1} \in \operatorname{span} E$ and $\left\|x_{2}\right\| \leq \varepsilon$, such that $x=x_{1}+x_{2}$. Since $y \longrightarrow \lambda(y) e$ is continuous for $e \in E$, so it is for $e \in \operatorname{span} E$. Hence, there is some neighbourhood $U^{\prime} \subseteq U$ of $y^{\prime}$, such that $\left\|\lambda(y) x_{1}-\lambda\left(y^{\prime}\right) x_{1}\right\| \leq \varepsilon$ for all $y \in U^{\prime}$. Consequently,

$$
\begin{aligned}
\left\|\lambda(y) x-\lambda\left(y^{\prime}\right) x\right\| & \leq\left\|\lambda(y) x_{1}-\lambda\left(y^{\prime}\right) x_{1}\right\|+\left\|\lambda(y) x_{2}-\lambda\left(y^{\prime}\right) x_{2}\right\| \\
& \leq\left\|\lambda(y) x_{1}-\lambda\left(y^{\prime}\right) x_{1}\right\|+\left(\|\lambda(y)\|+\left\|\lambda\left(y^{\prime}\right)\right\|\right)\left\|x_{2}\right\| \\
& \leq(2 c+1) \varepsilon
\end{aligned}
$$

for all $y \in U^{\prime}$. Hence, $y \longmapsto \lambda(y) x$ is continuous in $y^{\prime}$ for all $x \in X$. Since $y^{\prime}$ was arbitrary, we get the proof.

\section{B. Two Estimates}

Lemma B.1. Let $H$ be some Hilbert space and $N \in \mathbb{N}$. Moreover, let $A, A_{i}$ and $B_{i}$ be linear continuous operators on $H$, such that $\|A\| \leq 1$ and $\left\|B_{i}\right\| \leq 1$ for all $i=1, \ldots, N$. Then

$$
\left\|\prod_{i=1}^{N} A_{i} B_{i}-\prod_{i=1}^{N} A B_{i}\right\| \leq \prod_{i=1}^{N}\left(1+\left\|A_{i}-A\right\|\right)-1
$$


Proof. We have

$$
\begin{aligned}
& \left\|\prod_{i=1}^{N} A_{i} B_{i}-\prod_{i=1}^{N} A B_{i}\right\|=\left\|\prod_{i=1}^{N}\left(A+\left[A_{i}-A\right]\right) B_{i}-\prod_{i=1}^{N} A B_{i}\right\| \\
& \quad \leq \prod_{i=1}^{N}\left(\left\|A B_{i}\right\|+\left\|\left(A_{i}-A\right) B_{i}\right\|\right)-\prod_{i=1}^{N}\left\|A B_{i}\right\| \\
& \quad \leq \prod_{i=1}^{N}\left(\|A\|+\left\|A_{i}-A\right\|\right)-\prod_{i=1}^{N}\|A\| \\
& \quad \leq \prod_{i=1}^{N}\left(1+\left\|A_{i}-A\right\|\right)-1
\end{aligned}
$$

Lemma B.2. Let $\mathbf{G}$ be a connected, compact (hence linear) Lie group and let $\phi$ be an irreducible representation of $\mathbf{G}$ on $V_{\phi}$. Moreover, let $\left\{X_{i}\right\}_{i=1}^{n}$ be a basis of the Lie algebra $\mathfrak{g}$ of $\mathbf{G}$, such that $-\frac{1}{n} \sum_{i} \phi\left(X_{i}\right) \phi\left(X_{i}\right)$ is (up to the prefactor) the (quadratic) Casimir operator $C_{\phi}$ for $\phi$. Set

$$
c_{\phi, \mathfrak{g}}(t):=\frac{1}{2 n} \sum_{i=1}^{n}\left(\phi\left(\mathrm{e}^{t X_{i}}\right)+\phi\left(\mathrm{e}^{-t X_{i}}\right)\right) .
$$

Then, for all $t_{0}>0$, there is some $\eta\left(t_{0}\right)>0$ with

$$
\left\|\phi \otimes \bigotimes_{j=1}^{J}\left(c_{\phi, \mathfrak{g}}(t) \cdot \phi\right)-\mathrm{e}^{-\frac{1}{2} \lambda_{\phi} J t^{2}} \bigotimes_{j=0}^{J} \phi\right\|_{\infty} \leq \mathrm{e}^{\eta\left(t_{0}\right) J t^{4}}-1
$$

for all $|t|<t_{0}$ and all positive integers J. Here, $\lambda_{\phi}$ is the Casimir eigenvalue w.r.t. $\phi$, and $\|\cdot\|_{\infty}$ denotes the supremum norm in $\mathbf{G}^{J+1}$ induced by the standard operator norm $\|\cdot\|$ on $V_{\phi}$.

Proof. Let

$$
f_{1}(t):=c_{\phi, \mathfrak{g}}(t) \quad \text { and } \quad f_{2}(t):=\mathrm{e}^{-\frac{1}{2} \lambda_{\phi} t^{2}} \phi(\mathbf{1}) .
$$

We have $f_{1}(0)=\phi(\mathbf{1})=f_{2}(0)$. Next, $f_{1}^{\prime \prime}(0)=\frac{1}{n} \sum_{i=1}^{n} \phi\left(X_{i}\right) \phi\left(X_{i}\right)=-C_{\phi}$. Since $C_{\phi}=\lambda_{\phi} \phi(\mathbf{1})$, we have $f_{1}^{\prime \prime}(0)=-\lambda_{\phi} \phi(\mathbf{1})=f_{2}^{\prime \prime}(0)$. Since, moreover, the derivatives of odd degree vanish for both $f_{1}$ and $f_{2}$, the derivatives of $f_{1}$ and $f_{2}$ coincide up to degree 3 . Hence, for every $t_{0}>0$ there is some $\eta\left(t_{0}\right)>0$, such that $\left\|f_{1}(t)-f_{2}(t)\right\|<\eta\left(t_{0}\right) t^{4}$ for all $|t|<t_{0}$. Here, we used the analyticity of $f_{1}$ and $f_{2}$ on full $\mathbb{R}$.

Using Lemma B.1 and $\|\phi(g)\|=1$ (by unitarity of $\mathbf{G}$ ), we get

$$
\begin{gathered}
\left\|\phi\left(g_{0}\right) \prod_{j=1}^{J}\left(c_{\phi, \mathfrak{g}}(t) \phi\left(g_{j}\right)\right)-\mathrm{e}^{-\frac{1}{2} \lambda_{\phi} J t^{2}} \prod_{j=0}^{J} \phi\left(g_{j}\right)\right\| \\
\leq \prod_{j=1}^{J}\left(1+\left\|c_{\phi, \mathfrak{g}}(t)-\mathrm{e}^{-\frac{1}{2} \lambda_{\phi} t^{2}} \phi(\mathbf{1})\right\|\right)-1 \\
\leq \prod_{j=1}^{J}\left(1+\eta\left(t_{0}\right) t^{4}\right)-1 \\
\leq \mathrm{e}^{\eta\left(t_{0}\right) J t^{4}}-1
\end{gathered}
$$

for all $g_{0}, \ldots, g_{J} \in \mathbf{G}$ and all $|t|<t_{0}$. 


\section{C. "Bumpy" Stratified Isomorphisms}

Lemma C.1. Let $\tau_{1}$ and $\tau_{2}$ be real numbers with $\tau_{1}<\tau_{2}$. Moreover, let $0<\varepsilon<$ $\frac{1}{2}\left(\tau_{2}-\tau_{1}\right)$ and $a>0$. Finally, let $n \geq 2$ be an integer and define

$$
C:=\left[\tau_{1}-\varepsilon, \tau_{2}+\varepsilon\right] \times[-2 \varepsilon, 2 a+2 \varepsilon] \times B_{2 \varepsilon}^{n-2} \subseteq \mathbb{R}^{n},
$$

where $B_{r}^{m}$ is the ball around the origin in $m$ dimensions with radius $r$.

Then there is a stratified analytic isomorphism $\varphi$ of $\mathbb{R}^{n}$ with the following properties (see also Fig. 1 in Subsect. 6.4.1):

- $\varphi$ is the identity outside $C$;

- $\varphi$ changes the second (i.e., y-)coordinate only;

- $\varphi$ maps the first (i.e., $x$-) coordinate axis (restricted to $\left[\tau_{1}-\varepsilon, \tau_{2}+\varepsilon\right]$ ) to the union of straight lines connecting the points

$$
\left(\tau_{1}-\varepsilon, 0, \overrightarrow{0}\right),\left(\tau_{1}+\varepsilon, 2 a, \overrightarrow{0}\right),\left(\tau_{2}-\varepsilon, 2 a, \overrightarrow{0}\right), \text { and }\left(\tau_{2}+\varepsilon, 0, \overrightarrow{0}\right) .
$$

Proof. W.l.o.g. we may assume that $\tau:=\tau_{2}=-\tau_{1}$. Decompose $C$ into the 18 subsets $^{30}$

$$
G_{i j 0}:=G_{i j} \times B_{\varepsilon}^{n-2}
$$

and

$$
G_{i j+}:=G_{i j} \times\left(B_{2 \varepsilon}^{n-2} \backslash \text { int } B_{\varepsilon}^{n-2}\right)
$$

having overlapping boundaries (for the definition of $G_{i j}$, see Fig. 1). We are going to explicitly construct a diffeomorphism $\varphi$ mapping $G_{i j *}$ onto some $H_{i j *}$. Before stating the explicit formulae, we explain them verbally for $n=2$. $G_{11}$ is mapped to $H_{11}$, such that lines parallel to the $x$-axis are mapped to lines through $\left(x_{0}, y_{0}\right)$, whereas the line $x=-\tau-\varepsilon$ is preserved pointwise. The mapping between $G_{12}$ and $H_{12}$ simply makes (mutually parallel) sloped lines out of lines parallel to the $x$-axis. $G_{13}$ is mapped to $H_{13}$ similarly as $G_{11}$ to $H_{11}$. The maps $G_{2 i} \longrightarrow H_{2 i}$ map a line parallel to the $x$-axis again to such a line. The shift is completely determined by the shift on the left boundaries of the $G_{2 i}$. These, of course, are already given by maps of the right boundaries of $G_{1 i}$. The maps for $G_{3 i}$ will not be given explicitly. They just follow by the reflection symmetry w.r.t. $x=0$. The ideas above widely fix $\varphi$. We only have to take care of the matching conditions in the $\vec{z}$-directions. Here, we introduce a "fall-off" when $\|\vec{z}\|$ is in $[\varepsilon, 2 \varepsilon]$. For this, we define $g(\vec{z}):=\frac{1}{2}\left(1-\cos \left(\frac{\pi}{\varepsilon}\|\vec{z}\|\right)\right)$.

In the following, we will use that for any analytic function $h: \mathbb{R} \times \mathbb{R}^{n-2}$ and any $y_{0} \in \mathbb{R}$,

is invertible analytically on

$$
\begin{aligned}
& \varphi_{\text {aux }}: \mathbb{R}^{n} \longrightarrow \mathbb{R}^{n} \\
& (x, y, \vec{z}) \longmapsto\left(x, y+h(x, \vec{z})\left(y_{0}-y\right), \vec{z}\right)
\end{aligned}
$$

$$
U_{h}:=\{(x, y, \vec{z}) \mid h(x, \vec{z}) \neq 1\}
$$

by

$$
\begin{aligned}
& \varphi_{\text {aux }}^{-1}: U_{h} \quad \longrightarrow \quad \mathbb{R}^{n} . \\
& (x, y, \vec{z}) \longmapsto\left(x, \frac{y-h(x, \vec{z}) y_{0}}{1-h(x, \vec{z})}, \vec{z}\right) .
\end{aligned}
$$

Let us now state the diffeomorphism setting $y_{0}:=a+2 \varepsilon$ :

\footnotetext{
30 Of course, if $n=3$, there are 27 connected components, and for $n=2$ there are only 9 . We drop the corresponding cases here.
} 
- Define

and

$$
\left.\varphi_{110}: \underset{\mathbb{R}^{n}}{\longrightarrow} \stackrel{\mathbb{R}^{n}}{\longrightarrow}(x, y, \vec{z}) \longmapsto \frac{a}{\varepsilon} \frac{x+\tau+\varepsilon}{2 a+\varepsilon}\left(y_{0}-y\right), \vec{z}\right)
$$

$$
\begin{aligned}
\varphi_{11+}: & \mathbb{R}^{n} \\
(x, y, \vec{z}) & \longmapsto \\
\mathbb{R}^{n} & \left(x, y+g(\vec{z}) \frac{a}{\varepsilon} \frac{x+\tau+\varepsilon}{2 a+\varepsilon}\left(y_{0}-y\right), \vec{z}\right) .
\end{aligned}
$$

Both $\varphi_{110}$ and $\varphi_{11+}$ are analytically invertible on $x<-\tau+\varepsilon+\frac{\varepsilon^{2}}{a}$ and are the identity on $x=-\tau-\varepsilon$. Moreover, they coincide on $G_{110} \cap G_{11+}$. Finally, $\varphi_{11+}$ is the identity on $\|\vec{z}\|=2 \varepsilon$.

- Define

and

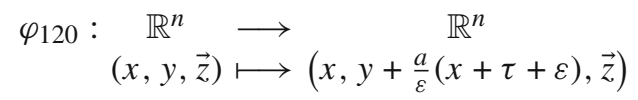

$$
\varphi_{12+}: \underset{\mathbb{R}^{n}}{\mathbb{R}_{(x, y, \vec{z})} \longmapsto}\left(x, y+g(\vec{z}) \frac{a}{\varepsilon}(x+\tau+\varepsilon), \vec{z}\right) .
$$

Both $\varphi_{120}$ and $\varphi_{12+}$ are analytically invertible on full $\mathbb{R}^{n}$, coincide on $G_{120} \cap G_{12+}$ and are the identity on $x=-\tau-\varepsilon$. In particular, observe that

$$
\varphi_{120}(-\tau-\varepsilon, y, \overrightarrow{0})=(-\tau-\varepsilon, y, \overrightarrow{0})
$$

and

$$
\varphi_{120}(-\tau+\varepsilon, y, \overrightarrow{0})=(-\tau+\varepsilon, y+2 a, \overrightarrow{0})
$$

i.e., $\varphi_{120}(-\tau, 0, \overrightarrow{0})=(-\tau, a, \overrightarrow{0})$.

- The maps $\varphi_{13 *}: \mathbb{R}^{n} \longrightarrow \mathbb{R}^{n}$ are defined analogously to the case of $\varphi_{11 *}$.

- The maps $\varphi_{2 i *}$ are given by

$$
\varphi_{2 i *}: \underset{\mathbb{R}^{n}}{\longrightarrow} \stackrel{\mathbb{R}^{n},}{\longrightarrow}\left(x, \operatorname{pr}_{y} \varphi_{1 i *}(-\tau+\varepsilon, y, \vec{z}), \vec{z}\right),
$$

where $\operatorname{pr}_{y}$ is the projection to the $y$-component.

- The remaining maps $\varphi_{3 i *}$ are defined using the reflection symmetry w.r.t. $x=0$.

One immediately checks that $\varphi: \mathbb{R}^{n} \longrightarrow \mathbb{R}^{n}$ defined by $\left.\varphi\right|_{G_{i j *}}:=\varphi_{i j *}$ and $\left.\varphi\right|_{\mathbb{R}^{n} \backslash C}:=\mathrm{id}$ is a well-defined stratified analytic isomorphism with the desired properties.

Open Access This article is distributed under the terms of the Creative Commons Attribution Noncommercial License which permits any noncommercial use, distribution, and reproduction in any medium, provided the original author(s) and source are credited.

\section{References}

1. Ashtekar, A.: New Hamiltonian formulation of general relativity. Phys. Rev. D 36, 1587-1602 (1987)

2. Ashtekar, A., Isham, C.J.: Representations of the holonomy algebras of gravity and nonabelian gauge theories. Class. Quant. Grav. 9, 1433-1468 (1992)

3. Ashtekar, A., Lewandowski, J.: Differential geometry on the space of connections via graphs and projective limits. J. Geom. Phys. 17, 191-230 (1995)

4. Ashtekar, A., Lewandowski, J.: Projective techniques and functional integration for gauge theories. J. Math. Phys. 36, 2170-2191 (1995)

5. Ashtekar, A., Lewandowski, J.: Quantum theory of geometry I: Area operators. Class. Quant. Grav. 14, A55-A82 (1997)

6. Ashtekar, A., Lewandowski, J.: Representation theory of analytic holonomy $C^{*}$ algebras. In: Baez, J.C. (ed.) Knots and Quantum Gravity (Riverside, CA, 1993), Oxford Lecture Series in Mathematics and its Applications 1, pp. 21-61, Oxford University Press, Oxford (1994) 
7. Baez, J.C., Sawin, S.: Diffeomorphism-invariant spin network states. J. Funct. Anal. 158, 253-266 (1998)

8. Baez, J.C., Sawin, S.: Functional integration on spaces of connections. J. Funct. Anal. 150, 1-26 (1997)

9. Bahr, B., Thiemann, T.: Automorphisms in loop quantum gravity. http://arxiv.org/list/0711.0373[gr-qc] (2007)

10. Bierstone, E., Milman, P.D.: Semianalytic and subanalytic sets. Publ. Math. IHES 67, 5-42 (1988)

11. Bratteli, O., Robinson, D.W.: Operator Algebras and Quantum Statistical Mechanics, vol. 2 (Equilibrium States, Models in Quantum Statistical Mechanics). Springer-Verlag, New York (1996)

12. Bratteli, O., Robinson, D.W.: Operator Algebras and Quantum Statistical Mechanics, vol. 1 ( $C^{*}$ - and $W^{*}$-Algebras, Symmetry Groups, Decomposition of States). Springer-Verlag, New York (1987)

13. Fleischhack, Ch.: Construction of generalized connections. http://arxiv.org/list/math-ph/0601005 (2006)

14. Fleischhack, Ch.: Hyphs and the Ashtekar-Lewandowski Measure. J. Geom. Phys. 45, 231-251 (2003)

15. Fleischhack, Ch.: Irreducibility of the Weyl Algebra in Loop Quantum Gravity. Phys. Rev. Lett. 97, 061302 (2006)

16. Fleischhack, Ch.: Mathematische und physikalische Aspekte verallgemeinerter Eichfeldtheorien im Ashtekarprogramm (Dissertation). Universität Leipzig (2001)

17. Fleischhack, Ch.: Proof of a Conjecture by Lewandowski and Thiemann. Commun. Math. Phys. 249, 331-352 (2004)

18. Fleischhack, Ch.: Regular connections among generalized connections. J. Geom. Phys. 47, 469-483 (2003)

19. Fleischhack, Ch.: Stratification of the Generalized Gauge Orbit Space. Commun. Math. Phys. 214, 607-649 (2000)

20. Gallagher, P.X.: Zeros of group characters. Math. Zeitschr. 87, 363-364 (1965)

21. Hardt, R.M.: Stratification of Real Analytic Mappings and Images. Invent. Math. 28, 193-208 (1975)

22. Huebsch, W., Morse, M.: Diffeomorphisms of manifolds. Rend. Circ. Mat. Palermo 11, 291-318 (1962)

23. Kamiński, W., Lewandowski, J., Bobieński, M.: Background independent quantizations: the scalar field I. Class. Quant. Grav. 23, 2761-2270 (2006)

24. Kamiński, W., Lewandowski, J., Okołów, A.: Background independent quantizations: the scalar field II. Class. Quant. Grav. 23, 5547-5586 (2006)

25. Lewandowski, J., Okołów, A.: Automorphism covariant representations of the holonomy-flux *-algebra. Class. Quant. Grav. 22, 657-680 (2005)

26. Lewandowski, J., Okołów, A., Sahlmann, H., Thiemann, T.: Uniqueness of diffeomorphism invariant states on holonomy-flux algebras. Commun. Math. Phys. 267, 703-733 (2006)

27. Lewandowski, J., Thiemann, T.: Diffeomorphism invariant quantum field theories of connections in terms of webs. Class. Quant. Grav. 16, 2299-2322 (1999)

28. Łojasiewicz, S.: On semi-analytic and subanalytic geometry. In: Panoramas of mathematics (Warszawa, 1992/1994), Banach Center Publications 34, pp. 89-104, Polish Academy of Sciences, Warszawa (1995)

29. Łojasiewicz, S.: Triangulation of semi-analytic sets. Ann. Scuola Norm. Sup. Pisa 18, 449-474 (1964)

30. Okołów, A., Lewandowski, J.: Diffeomorphism covariant representations of the holonomy-flux *-algebra. Class. Quant. Grav. 20, 3543-3568 (2003)

31. Pezzana, M.: Strong analytic triangulation for manifolds (Review of an article by Massimo Ferrarotti (Boll. Un. Mat. Ital. A 17, 79-84 (1980))). Math. Rev. 81e, 32012 (1981)

32. Sahlmann, H.: Some results concering the representation theory of the algebra underlying loop quantum gravity. http://arxiv.org/list/gr-qc/0207111 (2002)

33. Sahlmann, H.: When do measures on the space of connections support the triad operators of loop quantum gravity? http://arxiv.org/list/gr-qc/0207112 (2002)

34. Sahlmann, H., Thiemann, T.: Irreducibility of the Ashtekar-Isham-Lewandowski Representation. Class. Quant. Grav. 23, 4453-4472 (2006)

35. Sahlmann, H., Thiemann, T.: On the superselection theory of the weyl algebra for diffeomorphism invariant quantum gauge theories. http://arxiv.org/list/gr-qc/0302090 (2003)

36. Takesaki, M.: Theory of Operator Algebras I (Encyclopaedia of Mathematical Sciences 124). SpringerVerlag, Berlin (2002)

37. Whitehead, J.H.C.: On $C^{1}$-complexes. Annals of Math. 41, 809-824 (1940)

38. Whitney, H.: Geometric Integration Theory. Princeton University Press, Princeton, NJ (1957)

39. Zeidler, E. (Hrsg.): Taschenbuch der Mathematik, Bd. 1. Teubner-Verlag, Leipzig, Stuttgart (1996)

Communicated by Y. Kawahigashi 